\title{
COULOMB BRANCHES OF 3-DIMENSIONAL GAUGE THEORIES AND RELATED STRUCTURES
}

\author{
ALEXANDER BRAVERMAN AND MICHAEL FINKELBERG
}

\begin{abstract}
These are (somewhat informal) lecture notes for the CIME summer school "Geometric Representation Theory and Gauge Theory" in June 2018. In these notes we review the constructions and results of [BFN1, BFN2, BFN3] where a mathematical definition of Coulomb branches of $3 \mathrm{~d} N=4$ quantum gauge theories (of cotangent type) is given, and also present a framework for studying some further mathematical structures (e.g. categories of line operators in the corresponding topologically twisted theories) related to these theories.
\end{abstract}

\section{Introduction And First motivation: Symplectic DUAlity and A LITTLE BIT OF PHYSICS}

1.1. Symplectic singularities. Let $X$ be an algebraic variety over $\mathbb{C}$. We say that $X$ is singular symplectic (or $X$ has symplectic singularities) if

(1) $X$ is a normal Poisson variety;

(2) There exists a smooth dense open subset $U$ of $X$ on which the Poisson structure comes from a symplectic structure. We shall denote by $\omega$ the corresponding symplectic form.

(3) There exists a resolution of singularities $\pi: \widetilde{X} \rightarrow X$ such that $\pi^{*} \omega$ has no poles on $\widetilde{X}$.

This definition is due to A. Beauville, who showed that if condition (3) above holds for some $\tilde{X}$ then it holds for any resolution of $X$

1.2. Conical symplectic singularities. We say that $X$ is a conical symplectic singularity if in addition to (1)-(3) above the following conditions are satisfied:

(4) $X$ is affine;

(5) There exists a $\mathbb{C}^{\times}$-action on $X$ which contracts it to a point $x_{0} \in X$ and such that the form $\omega$ has positive weight.

We shall consider examples a little later.

1.3. Symplectic resolutions. By a symplectic resolutions we mean a morphism $\pi: \widetilde{X} \rightarrow X$ such that

(a) $X$ satisfies (1)-(5) above;

(b) $\widetilde{X}$ is smooth and $\pi$ is proper and birational and the action of $\mathbb{C}^{\times}$on $X$ extends to an action on $\widetilde{X}$. 
(c) $\pi^{*} \omega$ extends to a symplectic form on $\tilde{X}$.

Example. Let $\mathfrak{g}$ be a semi-simple Lie algebra over $\mathbb{C}$ and let $\mathcal{N}_{\mathfrak{g}} \subset \mathfrak{g}^{*}$ be its nilpotent cone. Let $\mathcal{B}$ denote the flag variety of $\mathfrak{g}$. Then the Springer map $\pi: T^{*} \mathcal{B} \rightarrow \mathcal{N}_{\mathfrak{g}}$ is proper and birational, so if we let $X=\mathcal{N}_{\mathfrak{g}}, \widetilde{X}=T^{*} \mathcal{B}$ we get a symplectic resolution.

1.4. The spaces $\mathfrak{t}_{X}$ and $\mathfrak{s}_{X}$. To any conical symplectic singularity $X$ one can associate two canonical vector spaces which we shall denote by $\mathfrak{t}_{X}$ and $\mathfrak{s}_{X}$. The space $\mathfrak{s}_{X}$ is just the Cartan subalgebra of the group of Hamiltonian automorphisms of $X$ commuting with the contracting $\mathbb{C}^{\times}$-action (which is an finite-dimensional algebraic group over $\mathbb{C}$ ). The space $\mathfrak{t}_{X}$ is trickier to define. First, assume that $X$ has a symplectic resolution $\tilde{X}$. Then $\mathfrak{t}_{X}=H^{2}(\widetilde{X}, \mathbb{C}$ ) (it follows from the results of Namikawa that $\mathfrak{t}_{X}$ is independent of the choice of $\left.\widetilde{X}\right)$. Moreover, $\mathfrak{t}_{X}$ also has another interpretation: there is a deformation $X$ of $X$ as a singular symplectic variety over the base $\mathfrak{t}_{X}$. The map $X \rightarrow \mathfrak{t}_{X}$ is smooth away from a finite union of hyperplanes in $\mathfrak{t}_{X}$.

If $X$ doesn't have have a symplectic resolution, Namikawa still defines the space $\mathfrak{t}_{X}$ and the above deformation; the only difference is that in this case $\mathcal{X}$ is no longer smooth over the generic point of $\mathfrak{t}_{X}$ (informally, one can say that $X$ is the deformation which makes $X$ "as smooth as possible" while staying in the class of symplectic varieties).

1.5. Some examples. Let $X$ be $\mathcal{N}_{\mathfrak{g}}$ as in the example above. Then $\mathfrak{s}_{X}$ is the Cartan subalgebra of $\mathfrak{g}$ and $\mathfrak{t}_{X}$ is its dual space. One may think that $\mathfrak{t}_{X}$ and $\mathfrak{s}_{X}$ always have the same dimension. However, it is not true already in the case $X=\mathbb{C}^{2 n}$. In this case $\mathfrak{s}_{X}$ has dimension $n$ and $\mathfrak{t}_{X}=0$.

Let now $X$ be a Kleinian surface singularity of type $A, D$ or $E$. In other words $X$ is isomorphic to $\mathbb{C}^{2} / \Gamma$ where $\Gamma$ is a finite subgroup of $S L(2, \mathbb{C})$. Thus $X$ has a unique singular point and it is known that $X$ has a symplectic resolution $\widetilde{X}$ with exceptional divisor formed by a tree of $\mathbb{P}^{1}$ 's whose intersection matrix is the above Cartan matrix of type $A, D$ or $E$. Thus the dimension of $\mathfrak{t}_{X}$ is the rank of this Cartan matrix. On the other hand, it is easy to show that $\mathfrak{s}_{X}$ is 1-dimensional if $\Gamma$ is of type $A$ and is equal to 0 otherwise.

1.6. The idea of symplectic duality. The idea of symplectic duality is this: ${ }^{1}$ often conical symplectic singularities come in "dual" pairs $\left(X, X^{*}\right)$ (the assignment $X \rightarrow X^{*}$ is by no means a functor; we just have a lot of interesting examples of dual pairs). What does it mean that $X$ and $X^{*}$ are dual? There is no formal definition; however, there are a lot of interesting properties that a dual pair must satisfy. The most straightforward one is this: we should have

$$
\mathfrak{t}_{X}=\mathfrak{s}_{X^{*}} \quad \mathfrak{s}_{X}=\mathfrak{t}_{X^{*}} .
$$

\footnotetext{
${ }^{1}$ The main ideas are due to T. Braden, A. Licata, N. Proudfoot and B. Webster.
} 
Other properties of dual pairs are more difficult to describe. For example, if both $X$ and $X^{*}$ have symplectic resolutions $\widetilde{X}$ and $\widetilde{X}^{*}$ then one should have

$$
\operatorname{dim} H^{*}(\widetilde{X}, \mathbb{C})=\operatorname{dim} H^{*}\left(\widetilde{X}^{*}, \mathbb{C}\right) .
$$

(However, these spaces are not supposed to be canonically isomorphic). We refer the reader to [BPW], [BLPW] for more details.

One of the purposes of these notes will be to provide a construction of a large class of symlectically dual pairs. Before we discuss what this class is, let us talk about some examples.

\subsection{Examples of symplectically dual spaces.}

1.7.1. Nilpotent cones. Let $X=\mathcal{N}_{\mathfrak{g}}$ and let $X^{*}=\mathcal{N}_{\mathfrak{g} \vee}$ where $\mathfrak{g}^{\vee}$ is the Langlands dual Lie algebra. This is supposed to be a symplectically dual pair.

1.7.2. Slodowy slices in type $A$. For partitions $\lambda \geq \mu$ of $n$, let $\mathcal{S}_{\mu}^{\lambda}$ be the intersection of the nilpotent orbit closure $\overline{\mathbb{O}}_{\lambda} \subset \mathfrak{g l}(n)$ with the Slodowy slice to the orbit $\mathbb{O}_{\mu}$. Then $\mathcal{S}_{\mu}^{\lambda}$ is dual to $\mathcal{S}_{\lambda^{t}}^{\mu^{t}}$.

1.7.3. Toric hyperkähler manifolds. Consider an exact sequence

$$
0 \rightarrow \mathbb{Z}^{d-n} \stackrel{\alpha}{\rightarrow} \mathbb{Z}^{d \stackrel{\beta}{\rightarrow}} \mathbb{Z}^{n} \rightarrow 0
$$

of the free based $\mathbb{Z}$-modules. It gives rise to a toric hyperkähler manifold $X$ [BiDa]. Then $X^{*}$ is the toric hyperkähler manifold associated to the dual exact sequence (Gale duality).

1.7.4. Uhlenbeck spaces. $\operatorname{Sym}^{a}\left(\mathbb{A}^{2} / \Gamma\right)^{\vee} \simeq \mathcal{U}_{G}^{a}\left(\mathbb{A}^{2}\right) / \mathbb{G}_{a}^{2}$ for a finite subgroup $\Gamma \subset$ $S L(2)$ corresponding by McKay to an almost simple simply connected simply laced Lie group $G$. Here $\mathbb{G}_{a}^{2}$ acts on $\mathbb{A}^{2}$ by translations, and hence it acts on $\mathcal{U}_{G}^{a}\left(\mathbb{A}^{2}\right)$ by the transport of structure.

1.8. $3 \mathrm{~d} \mathbf{N}=4$ quantum field theories and symplectic duality. One source of dual pairs $\left(X, X^{*}\right)$ comes from quantum field theory. We discuss this in more detail in Section 4; here we are just going to mention briefly the relevant notions.

Physicists have a notion of 3 -dimensional $\mathrm{N}=4$ super-symmetric quantum field theory. Any such theory $\mathcal{T}$ is supposed to have a well-defined moduli space of vacua $\mathcal{M}(\mathcal{T})$. This space is somewhat too complicated for our present discussion. Instead we are going to discuss some "easy" parts of this space. Namely, the above moduli space of vacua should have two special pieces called the Higgs and the Coulomb branch of the moduli space of vacua; we shall denote these by $\mathcal{M}_{H}(\mathcal{T})$ and $\mathcal{M}_{C}(\mathcal{T})$. They are supposed to be Poisson (generically symplectic) complex algebraic varieties (in fact, the don't even have to be algebraic but for simplicity we shall only consider examples when they are). They should also be hyper-kähler in some sense, but (to the best of our knowledge) this notion is not well-defined 
for singular varieties, we are going to ignore the hyper-kähler structure in these notes. But at least they are expected to be singular symplectic.

There is no mathematical classification of $3 \mathrm{~d} \mathrm{~N}=4$ theories. However, here is a class of examples. Let $G$ be a complex reductive algebraic group and let $\mathbf{M}$ be a symplectic representation of $G$; moreover we shall assume that the action of $G$ is Hamiltonian, i.e. that we have a well-defined moment map $\mu: \mathbf{M} \rightarrow \mathfrak{g}^{*}$ (this map can be fixed uniquely by requiring that $\mu(0)=0)$. Then to the pair $(G, \mathbf{M})$ one is supposed to associate a theory $\mathcal{T}(G, \mathbf{M})$. This theory is called gauge theory with gauge group $G$ and matter $\mathbf{M}$. Its Higgs branch is expected to be equal to $\mathbf{M} / / / G$ - the Hamiltonian reduction of $\mathbf{M}$ with respect to $G$. In particular, all Nakajima quiver varieties arise in this way (the corresponding theories are called quiver gauge theories).

What about the Coulomb branch of gauge theories? These are more tricky to define. Physicists have some expectations about those but no rigorous definition in general. For example, $\mathcal{M}_{C}(G, \mathbf{M})$ is supposed to be birationally isomorphic to $\left(T^{*} T^{\vee}\right) / W$. Here $T^{\vee}$ is the torus to dual the Cartan torus of $G$ and $W$ is the Weyl group. The above birational isomorphism should also preserve the Poisson structure. $^{2}$ In addition $\mathcal{M}_{C}(G, \mathbf{M})$ has a canonical $\mathbb{C}^{\times}$-action with respect to which the symplectic for has weight 2 . Unfortunately, it is not always conical but very often it is. Roughly speaking, to guarantee that $\mathcal{M}_{C}(G, \mathbf{M})$ is conical one needs that the representation $\mathbf{M}$ be "big enough" (for reasons not to be discussed here physicists call the corresponding gauge theories "good or ugly"). In the conical case physicists (cf. [CHZ]) produce a formula for the graded character of the algebra of functions on $\mathcal{M}_{C}(G, \mathbf{M})$. This formula is called "the monopole formula" (in a special case relevant for the purposes of these notes it is recalled in Subsection 5.3).

The idea is that at least in the conical case the pair $\left(\mathcal{M}_{H}(\mathcal{T}), \mathcal{M}_{C}(\mathcal{T})\right)$ should produce an example of a dual symplectic pair. One of the purposes of these notes (but not the only purpose) is to review the contents of the papers [BFN1, BFN2, BFN3] (joint with H. Nakajima) where a mathematical definition of the Coulomb branches $\mathcal{M}_{C}(G, \mathbf{M})$ ia given under an additional assumption (namely, we assume that $\mathbf{M}=T^{*} \mathbf{N}=\mathbf{N} \oplus \mathbf{N}^{*}$ for some representation $\mathbf{N}$ of $G$ - such theories are called gauge theories of cotangent type) and some further properties of Coulomb brancnes are studied. ${ }^{3}$ In this case we shall write $\mathcal{M}_{C}(G, \mathbf{N})$ instead of $\mathcal{M}_{C}(G, \mathbf{M})$. In loc. cit. it is defined as $\operatorname{Spec}\left(\mathbb{C}\left[\mathcal{M}_{C}(G, \mathbf{N})\right]\right)$ where $\mathbb{C}\left[\mathcal{M}_{C}(G, \mathbf{N})\right]$ is

\footnotetext{
${ }^{2}\left(T^{*} T^{\vee}\right) / W$ is actually the Coulomb branch of the corresponding classical field theory and the fact that the above birational isomorphism is not in general biregular means that "in the quantum theory the Coulomb branch acquires quantum corrections".

${ }^{3}$ The reader is also advised to consult the papers [Na2, Na3, Na4] by H. Nakajima. In particular, the papers [BFN1, BFN2, BFN3] are based on the ideas developed earlier in [Na2]. Also [Na3] contains a lot of interesting open problems in the subject most of which will not be addressed in these notes.
} 
some geometrically defined algebra over $\mathbb{C}$. The varieties $\mathcal{M}_{C}(G, \mathbf{N})$ are normal, affine, Poisson and generically symplectic. We expect that the they are actually singular symplectic, but we can't prove this in general. The main ingredient in the definition is the geometry of the affine Grassmannian $\operatorname{Gr}_{G}$ of $G$.

1.9. Remark about categorical symplectic duality. The following will never be used in the sequel, but we think it is important to mention it for the interested reader. Perhaps the most interesting aspect of symplectic duality is the categorical symplectic duality discussed in [BLPW]. Namely, in loc. cit. the authors explain that if both $X$ and $X^{*}$ have a symplectic resolution, then one can think about symplectic duality between them as Koszul duality between some version of category $\mathcal{O}$ over the quantization of the algebras $\mathbb{C}[X]$ and $\mathbb{C}\left[X^{*}\right]$. In fact, it is explained in [W] that a slightly weaker version of this statement can be formulated even when $X$ and $X^{*}$ do not have a symplectic resolution. This weaker statement is in fact proved in [W] for $\mathcal{M}_{H}(G, \mathbf{N})$ and $\mathcal{M}_{C}(G, \mathbf{N}$ ) (where the author uses the definition of $\mathcal{M}_{C}(G, \mathbf{N})$ from [BFN1]).

1.10. The plan. These notes are organized as follows. First, as was mentioned above the main geometric player in our construction of $\mathcal{M}_{C}(G, \mathbf{N})$ is the affine Grassmannian $\mathrm{Gr}_{G}$ of $G$. In Sections 2 and 3 we review some facts and constructions related to $\mathrm{Gr}_{G}$. Namely, in Section 2 we review the so called geometric Satake equivalence; in Section 3 we discuss an upgrade this construction: the derived geometric Satake equivalence. In Section 4 we discuss some general expectations about $3 \mathrm{~d} \mathrm{~N}=4$ theories and in Section 5 we give a definition of the varieties $\mathcal{M}_{C}(G, \mathbf{N})$. Section 6 is devoted to the example of quiver gauge theories; in particular, for finite quivers of $A D E$ type we identify the Coulomb branches with certain (generalized) slices in the affine Grassmannian of the corresponding group of $A D E$-type. Finally, in Section 7 we discuss some conjectural categorical structures related to the topologically twisted version of gauge theories of cotangent type (we have learned the main ideas of this Section from T. Dimofte, D. Gaiotto, J. Hilburn and P. Yoo).

1.11. Acknowledgements. We are greatly indebted to our coauthor H. Nakajima who taught us everything we know about Coulomb branches of $3 \mathrm{~d} N=4$ gauge theories and to the organizers of the CIME summer school "Geometric Representation Theory and Gauge Theory" in June 2018, for which these notes were written. In addition we would like to thank T. Dimofte, D. Gaiotto, J. Hilburn and P. Yoo for their patient explanations of various things (in particular, as was mentioned above the main idea of Section 7 is due to them). Also, we are very grateful to R. Bezrukavnikov, K. Costello, D. Gaitsgory and S. Raskin for their help with many questions which arose during the preparation of these notes. M.F. was partially supported by the Russian Academic Excellence Project '5-100'. 


\section{Geometric Satake}

2.1. Overview. Let $\mathcal{O}$ denote the formal power series ring $\mathbb{C}[[z]]$, and let $\mathcal{K}$ denote its fraction field $\mathbb{C}((z))$. Let $G$ be a reductive complex algebraic group with a Borel and a Cartan subgroup $G \supset B \supset T$, and with the Weyl group $W$ of $(G, T)$. Let $\Lambda$ be the coweight lattice, and let $\Lambda^{+} \subset \Lambda$ be the submonoid of dominant coweights. Let also $\Lambda_{+} \subset \Lambda$ be the submonoid spanned by the simple coroots $\alpha_{i}, i \in I$. We denote by $G^{\vee} \supset T^{\vee}$ the Langlands dual group, so that $\Lambda$ is the weight lattice of $G^{\vee}$.

The affine Grassmannian $\mathrm{Gr}_{G}=G_{\mathcal{K}} / G_{\mathcal{O}}$ is an ind-projective scheme, the union

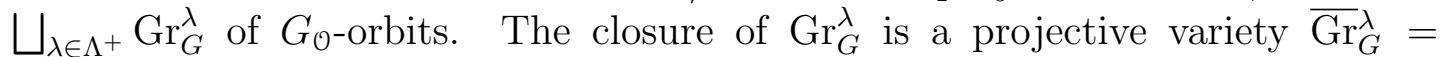
$\bigsqcup_{\mu \leq \lambda} \operatorname{Gr}_{G}^{\mu}$. The fixed point set $\operatorname{Gr}_{G}^{T}$ is naturally identified with the coweight lattice $\Lambda$; and $\mu \in \Lambda$ lies in $\operatorname{Gr}_{G}^{\lambda}$ iff $\mu \in W \lambda$.

One of the cornerstones of the Geometric Langlands Program initiated by $\mathrm{V}$. Drinfeld is an equivalence $\mathbb{S}$ of the tensor category $\operatorname{Rep}\left(G^{\vee}\right)$ and the category $\operatorname{Perv}_{G_{\mathcal{O}}}\left(\mathrm{Gr}_{G}\right)$ of $G_{\mathcal{O}}$-equivariant perverse constructible sheaves on $\mathrm{Gr}_{G}$ equipped with a natural monoidal convolution structure $\star$ and a fiber functor $H^{\bullet}\left(\mathrm{Gr}_{G},-\right)$ [Lus, Gi1, BD, MV]. It is a categorification of the classical Satake isomorphism between $K\left(\operatorname{Rep}\left(G^{\vee}\right)\right)=\mathbb{C}\left[T^{\vee}\right]^{W}$ and the spherical affine Hecke algebra of $G$. The geometric Satake equivalence $\mathbb{S}$ sends an irreducible $G^{\vee}$-module $V^{\lambda}$ with highest weight $\lambda$ to the Goresky-MacPherson sheaf $\operatorname{IC}\left(\overline{\operatorname{Gr}}_{G}^{\lambda}\right)$.

In order to construct a commutativity constraint for $\left(\operatorname{Perv}_{G_{\mathcal{O}}}\left(\operatorname{Gr}_{G}\right), \star\right)$, Beilinson and Drinfeld introduced a relative version $\operatorname{Gr}_{G, B D}$ of the Grassmannian over the Ran space of a smooth curve $X$, and a fusion monoidal structure $\Psi$ on $\operatorname{Perv}_{G_{\mathcal{O}}}\left(\mathrm{Gr}_{G}\right)$ (isomorphic to $\star$ ). One of the main discoveries of [MV] was a $\Lambda$ grading of the fiber functor $H^{\bullet}\left(\mathrm{Gr}_{G}, \mathcal{F}\right)=\bigoplus_{\lambda \in \Lambda} \Phi_{\lambda}(\mathcal{F})$ by the hyperbolic stalks at $T$-fixed points. For a $G^{\vee}$-module $V$, its weight space $V_{\lambda}$ is canonically isomorphic to the hyperbolic stalk $\Phi_{\lambda}(\mathbb{S} V)$.

Various geometric structures of a perverse sheaf $\mathbb{S} V$ reflect some fine representation theoretic structures of $V$, such as Brylinski-Kostant filtration and the action of dynamical Weyl group, see [GiRi]. One of the important technical tools of studying Perv $G_{\mathcal{O}}\left(\mathrm{Gr}_{G}\right)$ is the embedding $\mathrm{Gr}_{G} \hookrightarrow \mathbf{G r}_{G}$ into Kashiwara infinite type scheme $\mathbf{G r}_{G}=G_{\mathbb{C}\left(\left(z^{-1}\right)\right)} / G_{\mathbb{C}[z]}[\mathrm{Ka}, \mathrm{KT}]$. The quotient $G_{\mathbb{C}\left[\left[z^{-1}\right]\right]} \backslash \mathbf{G r}_{G}$ is the moduli stack $\operatorname{Bun}_{G}\left(\mathbb{P}^{1}\right)$ of $G$-bundles on the projective line $\mathbb{P}^{1}$. The $G_{\mathbb{C}\left[\left[z^{-1}\right]\right]^{-o r b i t s}}$ on $\mathbf{G r}_{G}$ are of finite codimension; they are also numbered by the dominant coweights of $G$, and the image of an orbit $\operatorname{Gr}_{G}^{\lambda}$ in $\operatorname{Bun}_{G}\left(\mathbb{P}^{1}\right)$ consists of $G$-bundles of isomorphism type $\lambda[\mathrm{Gr}]$. The stratifications $\mathrm{Gr}_{G}=\bigsqcup_{\lambda \in \Lambda^{+}} \operatorname{Gr}_{G}^{\lambda}$ and $\mathbf{G r}_{G}=\bigsqcup_{\lambda_{\in \Lambda^{+}}} \mathbf{G r}_{G}^{\lambda}$ are transversal, and their intersections and various generalizations thereof will play an important role later on.

More precisely, we denote by $K_{1}$ the first congruence subgroup of $G_{\mathbb{C}\left[\left[z^{-1}\right]\right]}$ : the kernel of the evaluation projection $\mathrm{ev}_{\infty}: G_{\mathbb{C}\left[\left[z^{-1}\right]\right]} \rightarrow G$. The transversal slice $\mathcal{W}_{\mu}^{\lambda}$ 
(resp. $\overline{\mathcal{W}}_{\mu}^{\lambda}$ ) is defined as the intersection of $\operatorname{Gr}_{G}^{\lambda}$ (resp. $\overline{\operatorname{Gr}}_{G}^{\lambda}$ ) and $K_{1} \cdot \mu$ in $\mathbf{G r}_{G}$. It is known that $\overline{\mathcal{W}}_{\mu}^{\lambda}$ is nonempty iff $\mu \leq \lambda$, and $\operatorname{dim} \overline{\mathcal{W}}_{\mu}^{\lambda}$ is an affine irreducible variety of dimension $\left\langle 2 \rho^{\vee}, \lambda-\mu\right\rangle$. Following an idea of I. Mirković, [KWY] proved that $\overline{\mathcal{W}}_{\mu}^{\lambda}=\bigsqcup_{\mu \leq \nu \leq \lambda} \mathcal{W}_{\mu}^{\nu}$ is the decomposition of $\overline{\mathcal{W}}_{\mu}^{\lambda}$ into symplectic leaves of a natural Poisson structure.

2.2. Hyperbolic stalks. Let $N$ denote the unipotent radical of the Borel $B$, and let $N_{-}$stand for the unipotent radical of the opposite Borel $B_{-}$. For a coweight $\nu \in \Lambda=\mathrm{Gr}_{G}^{T}$, we denote by $S_{\nu} \subset \mathrm{Gr}_{G}$ (resp. $T_{\nu} \subset \mathrm{Gr}_{G}$ ) the orbit of $N(\mathcal{K})$ (resp. of $N_{-}(\mathcal{K})$. The intersections $S_{\nu} \cap \overline{\mathrm{Gr}}_{G}^{\lambda}$ (resp. $T_{\nu} \cap \overline{\mathrm{Gr}}_{G}^{\lambda}$ ) are the attractors (resp. repellents) of $\mathbb{C}^{\times}$acting via its homomorphism $2 \rho$ to the Cartan torus $T \curvearrowright \overline{\mathrm{Gr}}_{G}^{\lambda}: S_{\nu} \cap \overline{\mathrm{Gr}}_{G}^{\lambda}=\left\{x \in \overline{\mathrm{Gr}}_{G}^{\lambda}: \lim _{c \rightarrow 0} 2 \rho(c) \cdot x=\nu\right\}$ and $T_{\nu} \cap \overline{\mathrm{Gr}}_{G}^{\lambda}=\{x \in$ $\left.\overline{\mathrm{Gr}}_{G}^{\lambda}: \lim _{c \rightarrow \infty} 2 \rho(c) \cdot x=\nu\right\}$. Going to the limit $\mathrm{Gr}_{G}=\lim _{\lambda \in \Lambda^{+}} \overline{\mathrm{Gr}}_{G}^{\lambda}, S_{\nu}$ (resp. $T_{\nu}$ ) is the attractor (resp. repellent) of $\nu$ in $\mathrm{Gr}_{G}$. We denote by $r_{\nu,+}$ (resp. $r_{\nu,-}$ ) the locally closed embedding $S_{\nu} \hookrightarrow \operatorname{Gr}_{G}$ (resp. $T_{\nu} \hookrightarrow \mathrm{Gr}_{G}$ ). We also denote by $\iota_{\nu,+}$ (resp. $\left.\iota_{\nu,-}\right)$ the closed embedding of the point $\nu$ into $S_{\nu}$ (resp. into $T_{\nu}$ ). The following theorem is proved in [Br, DG].

Theorem 2.3. There is a canonical isomorphism of functors $\iota_{\nu,+}^{*} r_{\nu,+}^{!} \simeq$ $\iota_{\nu,-}^{!} r_{\nu,-}^{*}: D_{G_{\mathcal{O}}}^{b}\left(\operatorname{Gr}_{G}\right) \rightarrow D^{b}$ (Vect).

Definition 2.4. For a sheaf $\mathcal{F} \in D_{G_{\mathcal{O}}}^{b}\left(\mathrm{Gr}_{G}\right)$ we define its hyperbolic stalk at $\nu$ as $\Phi_{\nu}(\mathcal{F}):=\iota_{\nu,+}^{*} r_{\nu,+}^{!} \mathcal{F} \simeq \iota_{\nu,-}^{!} r_{\nu,-}^{*} \mathcal{F}$.

The following dimension estimate due to $[\mathrm{MV}]$ is crucial for the geometric Satake.

Lemma 2.5. (a) $S_{\nu} \cap \mathrm{Gr}_{G}^{\lambda} \neq \varnothing$ iff $T_{\nu} \cap \mathrm{Gr}_{G}^{\lambda} \neq \varnothing$ iff $\nu$ has nonzero multiplicity in the irreducible $G^{\vee}$-module $V^{\lambda}$ with highest weight $\lambda$. This is also equivalent to $\nu \in \overline{\mathrm{Gr}}_{G}^{\lambda}$.

(b) The nonempty intersection $S_{\nu} \cap \overline{\mathrm{Gr}}_{G}^{\lambda}$ is equidimensional of dimension $\langle\nu+$ $\left.\lambda, \rho^{v}\right\rangle$.

(c) The nonempty intersection $T_{\nu} \cap \overline{\mathrm{Gr}}_{G}^{\lambda}$ is equidimensional of dimension $\langle\nu+$ $\left.w_{0} \lambda, \rho^{\vee}\right\rangle$. Here $w_{0}$ is the longest element of the Weyl group $W$.

Corollary 2.6. (a) For $\mathcal{F} \in \operatorname{Perv}_{G_{\mathcal{O}}}\left(\mathrm{Gr}_{G}\right)$, the hyperbolic stalk $\Phi_{\nu}(\mathcal{F})$ is concentrated in degree $\left\langle\nu, 2 \rho^{v}\right\rangle$.

(b) There is a canonical direct sum decomposition $H^{\bullet}\left(\mathrm{Gr}_{G}, \mathcal{F}\right)=\bigoplus_{\nu \in \Lambda} \Phi_{\nu}(\mathcal{F})$.

(c) The functor $H^{\bullet}\left(\mathrm{Gr}_{G},-\right): \operatorname{Perv}_{G_{\mathcal{O}}}\left(\mathrm{Gr}_{G}\right) \rightarrow \mathrm{Vect}^{\mathrm{gr}}$, as well as its upgrade $\bigoplus_{\nu \in \Lambda} \Phi_{\nu}: \operatorname{Perv}_{G_{\mathcal{O}}}\left(\operatorname{Gr}_{G}\right) \rightarrow \operatorname{Rep}\left(T^{\vee}\right)$, is exact and conservative.

2.7. Convolution. We have the following basic diagram:

$$
\mathrm{Gr}_{G} \times \mathrm{Gr}_{G} \stackrel{p}{\leftarrow} G_{\mathcal{K}} \times \mathrm{Gr}_{G} \stackrel{q}{\rightarrow} \operatorname{Gr}_{G} \widetilde{\times} \mathrm{Gr}_{G} \stackrel{m}{\rightarrow} \operatorname{Gr}_{G} .
$$


Here $\operatorname{Gr}_{G} \widetilde{\times} \mathrm{Gr}_{G}=G_{\mathcal{K}} \stackrel{G_{\mathcal{O}}}{\times} \operatorname{Gr}_{G}=\left(G_{\mathcal{K}} \times \mathrm{Gr}_{G}\right) /\left((g, x) \sim\left(g h^{-1}, h x\right), h \in G_{\mathcal{O}}\right)$. Furthermore, $p$ is the projection on the first factor and identity on the second factor, and the composition $m \circ q$ is the action morphism $G_{\mathcal{K}} \times \mathrm{Gr}_{G} \rightarrow \mathrm{Gr}_{G}$ (which clearly factors through $\left.G_{\mathcal{K}} \stackrel{G_{\mathcal{O}}}{\times} \mathrm{Gr}_{G}\right)$.

Definition 2.8. Given $\mathcal{F}_{1}, \mathcal{F}_{2} \in D_{G_{\mathcal{O}}}^{b}\left(\mathrm{Gr}_{G}\right)$, their convolution $\mathcal{F}_{1} \star \mathcal{F}_{2} \in D_{G_{\mathcal{O}}}^{b}\left(\mathrm{Gr}_{G}\right)$ is defined as $\mathcal{F}_{1} \star \mathcal{F}_{2}:=m_{*}\left(\mathcal{F}_{1} \widetilde{\otimes} \mathcal{F}_{2}\right)$, where $\mathcal{F}_{1} \widetilde{\otimes} \mathcal{F}_{2}$ is the descent of $p^{*}\left(\mathcal{F}_{1} \otimes \mathcal{F}_{2}\right)$, that is $q^{*}\left(\mathcal{F}_{1} \widetilde{\otimes} \mathcal{F}\right)=p^{*}\left(\mathcal{F}_{1} \otimes \mathcal{F}_{2}\right)$.

The next lemma is due to [Lus, MV]. It follows from the stratified semismallness of $m: \overline{\mathrm{Gr}}_{G}^{\lambda, \mu}:=p^{-1}\left(\overline{\mathrm{Gr}}_{G}^{\lambda}\right) \stackrel{G_{\mathcal{O}}}{\times} \overline{\mathrm{Gr}}_{G}^{\mu} \rightarrow \overline{\mathrm{Gr}}_{G}^{\lambda+\mu}$. Here $\overline{\mathrm{Gr}}_{G}^{\lambda, \mu}$ is stratified by the union of $\operatorname{Gr}_{G}^{\nu, \theta}:=p^{-1}\left(\operatorname{Gr}_{G}^{\nu}\right) \stackrel{G_{\mathcal{O}}}{\times} \operatorname{Gr}_{G}^{\theta}$ over $\nu \leq \lambda, \theta \leq \mu$. The stratified semismallness in turn follows from the dimension estimate of Lemma 2.5.

Lemma 2.9. Given $\mathcal{F}_{1}, \mathcal{F}_{2} \in \operatorname{Perv}_{G_{\mathcal{O}}}\left(\mathrm{Gr}_{G}\right)$, their convolution $\mathcal{F}_{1} \star \mathcal{F}_{2}$ lies in $\operatorname{Perv}_{G_{\mathcal{O}}}\left(\mathrm{Gr}_{G}\right)$ as well.

In order to define a commutativity constraint for $\star$, we will need an equivalent construction of the monoidal structure on $\operatorname{Perv}_{G_{\mathcal{O}}}\left(\mathrm{Gr}_{G}\right)$ via fusion due to V. Drinfeld.

2.10. Fusion. Let $X$ be a smooth curve, e.g. $X=\mathbb{A}^{1}$. We have the following basic diagram:

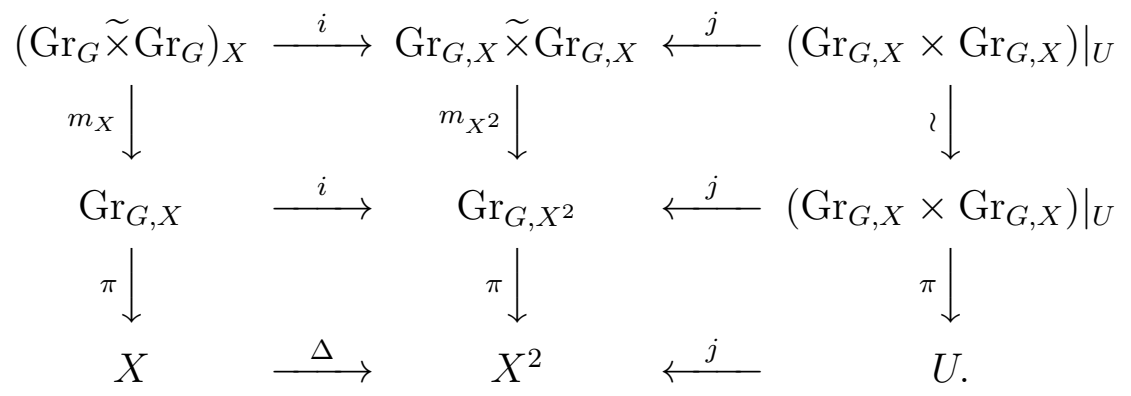

Here $U \hookrightarrow X^{2}$ is the open embedding of the complement to the diagonal $\Delta_{X} \hookrightarrow X^{2}$. Furthermore, for $n \in \mathbb{N}, \operatorname{Gr}_{G, X^{n}}$ is the moduli space of the following data: $\left\{\left(x_{1}, \ldots, x_{n}\right) \in X^{n}, \mathcal{P}_{G}, \tau\right\}$, where $\mathcal{P}_{G}$ is a $G$-bundle on $X$, and $\tau$ is a trivialization of $\mathcal{P}_{G}$ on $X \backslash\left\{x_{1}, \ldots, x_{n}\right\}$. The projection $\pi: \operatorname{Gr}_{G, X^{n}} \rightarrow X^{n}$ forgets the data of $\mathcal{P}_{G}$ and $\tau$. Note that $\left.\left.\operatorname{Gr}_{G, X^{2}}\right|_{U} \simeq\left(\operatorname{Gr}_{G, X} \times \operatorname{Gr}_{G, X}\right)\right|_{U}$, while $\left.\mathrm{Gr}_{G, X^{2}}\right|_{\Delta_{X}} \simeq \operatorname{Gr}_{G, X}$. Furthermore, $\mathrm{Gr}_{G, X} \widetilde{\times} \mathrm{Gr}_{G, X}$ is the moduli space of the following data: $\left\{\left(x_{1}, x_{2}\right) \in X^{2}, \mathcal{P}_{G}^{1}, \mathcal{P}_{G}^{2}, \tau, \sigma\right\}$, where $\mathcal{P}_{G}^{1}, \mathcal{P}_{G}^{2}$ are $G$-bundles on $X ; \sigma:\left.\left.\mathcal{P}_{G}^{1}\right|_{X \backslash x_{2}} \stackrel{\sim}{\sim} \mathcal{P}_{G}^{2}\right|_{X \backslash x_{2}}$, and $\tau$ is a trivialization of $\mathcal{P}_{G}^{1}$ on $X \backslash x_{1}$. Note that $\left.\left.\left(\operatorname{Gr}_{G, X} \widetilde{\times} \operatorname{Gr}_{G, X}\right)\right|_{U} \simeq\left(\operatorname{Gr}_{G, X} \times \operatorname{Gr}_{G, X}\right)\right|_{U}$, while $\left.\left(\operatorname{Gr}_{G, X} \widetilde{\times} \operatorname{Gr}_{G, X}\right)\right|_{\Delta_{X}}$ is fibered over $X$ with fibers isomophic to $\operatorname{Gr}_{G} \widetilde{\times} \mathrm{Gr}_{G}$. Finally, $m_{X^{2}}: \mathrm{Gr}_{G, X} \widetilde{\times} \mathrm{Gr}_{G, X} \rightarrow \operatorname{Gr}_{G, X^{2}}$ 
takes $\left(x_{1}, x_{2}, \mathcal{P}_{G}^{1}, \mathcal{P}_{G}^{2}, \tau, \sigma\right)$ to $\left(x_{1}, x_{2}, \mathcal{P}_{G}^{2}, \tau^{\prime}\right)$ where $\tau^{\prime}=\left.\sigma \circ \tau\right|_{X \backslash\left\{x_{1}, x_{2}\right\}}$. All the squares in the above diagram are cartesian. The stratified semismallness property of the convolution morphism $m$ used in the proof of Lemma 2.9 implies the stratified smallness property of the relative convolution morphism $m_{X^{2}}$.

Now given $\mathcal{F}_{1}, \mathcal{F}_{2} \in D_{G_{\mathcal{O}}}^{b}\left(\mathrm{Gr}_{G}\right)$, we can define the constructible complexes $\mathcal{F}_{1, X}, \mathcal{F}_{2, X}$ on $\operatorname{Gr}_{G, X}$ smooth over $X$, and by descent similarly to Section 2.7, a constructible complex $\mathcal{F}_{1, X} \widetilde{\otimes} \mathcal{F}_{2, X}$ on $\operatorname{Gr}_{G, X} \widetilde{\times} \mathrm{Gr}_{G, X}$ smooth over $X^{2}$. Note that $\left.\left(\mathcal{F}_{1, X} \widetilde{\otimes} \mathcal{F}_{2, X}\right)\right|_{U}=\left.\left(\mathcal{F}_{1, X} \otimes \mathcal{F}_{2, X}\right)\right|_{U}$. For simplicity, let us take $X=\mathbb{A}^{1}$. Then by the proper base change for nearby cycles $\Psi_{x_{1}-x_{2}} m_{X^{2} *}\left(\mathcal{F}_{1, X} \widetilde{\otimes} \mathcal{F}_{2, X}\right)$ on $\operatorname{Gr}_{G, X^{2}}$ we deduce $\left(\mathcal{F}_{1} \star \mathcal{F}_{2}\right)_{X}=\left.\Psi_{x_{1}-x_{2}}\left(\mathcal{F}_{1, X} \otimes \mathcal{F}_{2, X}\right)\right|_{U}$. The RHS being manifestly symmetric, we deduce the desired commutativity constraint for the convolution product $\star$.

Also, the above smoothness of $\mathcal{F}_{1, X} \widetilde{\otimes} \mathcal{F}_{2, X}$ over $X^{2}$ implies that $\pi_{*} m_{X^{2} *}\left(\mathcal{F}_{1, X} \widetilde{\otimes} \mathcal{F}_{2, X}\right)$ is a constant sheaf on $X^{2}$. Since its diagonal stalks are $H^{\bullet}\left(\mathrm{Gr}_{G}, \mathcal{F}_{1} \star \mathcal{F}_{2}\right)$, and the off-diagonal stalks are $H^{\bullet}\left(\mathrm{Gr}_{G}, \mathcal{F}_{1}\right) \otimes H^{\bullet}\left(\mathrm{Gr}_{G}, \mathcal{F}_{2}\right)$, we obtain that the cohomology functor $\operatorname{Perv}_{G_{\mathcal{O}}}\left(\mathrm{Gr}_{G}\right) \rightarrow$ Vect is a tensor functor: $H^{\bullet}\left(\mathrm{Gr}_{G}, \mathcal{F}_{1} \star \mathcal{F}_{2}\right) \stackrel{\sim}{\longrightarrow} H^{\bullet}\left(\mathrm{Gr}_{G}, \mathcal{F}_{1}\right) \otimes H^{\bullet}\left(\mathrm{Gr}_{G}, \mathcal{F}_{2}\right)$.

Corollary 2.11. The abelian category $\operatorname{Perv}_{G_{\mathcal{O}}}\left(\mathrm{Gr}_{G}\right)$ is equipped with a symmetric monoidal structure $\star$ and a fiber functor $H^{\bullet}\left(\mathrm{Gr}_{G},-\right)$.

By Tannakian formalism, the tensor category $\operatorname{Perv}_{G_{\mathcal{O}}}\left(\mathrm{Gr}_{G}\right)$ must be equivalent to $\operatorname{Rep}\left(G^{\prime}\right)$ for a proalgebraic group $G^{\prime}$. It remains to identify $G^{\prime}$ with the Langlands dual group $G^{\vee}$. From the semisimplicity of $\operatorname{Perv}_{G_{\mathcal{O}}}\left(\operatorname{Gr}_{G}\right)$, the group $G^{\prime}$ must be reductive. The upgraded fiber functor $\bigoplus_{\nu \in \Lambda} \Phi_{\nu}: \operatorname{Perv}_{G_{\mathcal{O}}}\left(\operatorname{Gr}_{G}\right) \rightarrow \operatorname{Rep}\left(T^{\vee}\right)$ is tensor since the nearby cycles commute with hyperbolic stalks by [Na1, Proposition 5.4.1.(2)]. Hence we obtain a homomorphism $T^{\vee} \hookrightarrow G^{\prime}$. Now it is easy to identify $G^{\prime}$ with $G^{\vee}$ using Lemma 2.5(a). We have proved

Theorem 2.12. There is a tensor equivalence $\mathbb{S}: \operatorname{Rep}\left(G^{\vee}\right), \otimes \stackrel{\sim}{\longrightarrow} \operatorname{Perv}_{G_{\mathcal{O}}}\left(\operatorname{Gr}_{G}\right)$, .

\section{Derived Geometric Satake}

In this section we extend the algebraic description of $\operatorname{Perv}_{G_{\mathcal{O}}}\left(\mathrm{Gr}_{G}\right)$ to an algebraic description of the equivariant derived category $D_{G_{\mathcal{O}} \rtimes \mathbb{C} \times}\left(\operatorname{Gr}_{G}\right)$.

3.1. Asymptotic Harish-Chandra bimodules. First we develop the necessary algebraic machinery. Let $U=U\left(\mathfrak{g}^{\vee}\right)$ be the universal enveloping algebra, and let $U_{\hbar}$ be the graded enveloping algebra, i.e. the graded $\mathbb{C}[\hbar]$-algebra generated by $\mathfrak{g}^{\vee}$ with relations $x y-y x=\hbar[x, y]$ for $x, y \in \mathfrak{g}^{\vee}$ (thus $U_{\hbar}$ is obtained from $U$ by the Rees construction producing a graded algebra from the filtered one). The adjoint action extends to the action of $G^{\vee}$ on $U_{\hbar}$. 
We consider the category $\mathcal{H} \mathcal{C}_{\hbar}$ of graded modules over $U_{\hbar}^{2}:=U_{\hbar} \otimes_{\mathbb{C}[\hbar]} U_{\hbar} \simeq$ $U_{\hbar} \rtimes U$ equipped with an action of $G^{\vee}$ (denoted $\beta: G^{\vee} \times M \rightarrow M$ ) satisfying the following conditions:

(a) The action $U_{\hbar}^{2} \otimes M \rightarrow M$ is $G^{\vee}$-equivariant;

(b) for any $x \in \mathfrak{g}^{\vee}$, the action of $x \otimes 1+1 \otimes x \in U_{\hbar}^{2}$ coincides with the action of $\hbar \cdot d \beta(x)$;

(c) the module $M$ is finitely generated as a $U_{\hbar} \otimes 1$-module (equivalently, as a $1 \otimes U_{\hbar}$-module).

The restriction from $U_{\hbar}^{2}$ to $U_{\hbar} \otimes 1$ gives an equivalence of $\mathcal{H} \bigodot_{\hbar}$ with the category of $G^{\vee}$-modules equipped with a $G^{\vee}$-equivariant $U_{\hbar}$-action.

3.1.1. Example: free Harish-Chandra bimodules. Let $V \in \operatorname{Rep}\left(G^{\vee}\right)$. We define a free Harish-Chandra bimodule $\operatorname{Fr}(V)=U_{\hbar} \otimes V$ with the $G^{\vee}$-action $g(y \otimes v)=$ $\operatorname{Ad}_{g}(y) \otimes g(v)$ and the $U_{\hbar}^{2}$-action $(x \otimes u)(y \otimes v)=x y u \otimes v+\hbar x y \otimes u(v)$, where $x, u \in \mathfrak{g}^{\vee} \subset U_{\hbar}$. Thus, $\operatorname{Fr}(V)$ is the induction of $V$ (the left adjoint functor to the restriction res: $\mathcal{H} \mathcal{C}_{\hbar} \rightarrow \operatorname{Rep}\left(G^{\vee}\right)$ ). This is a projective object of the category $\mathcal{H} \bigodot_{\hbar}$. We will denote by $\mathcal{H} \bigodot_{\hbar}^{\text {fr }}$ the full subcategory of $\mathcal{H} \bigodot_{\hbar}$ formed by all the free Harish-Chandra bimodules.

3.2. Kostant-Whittaker reduction. We also consider the subalgebra $U_{\hbar}^{2}\left(\mathfrak{n}_{-}^{\vee}\right)=U_{\hbar}\left(\mathfrak{n}_{-}^{\vee}\right) \rtimes U\left(\mathfrak{n}_{-}^{\vee}\right) \subset U_{\hbar}^{2}$. We fix a regular character $\psi: U_{\hbar}\left(\mathfrak{n}_{-}^{\vee}\right) \rightarrow \mathbb{C}[\hbar]$ taking value 1 at each generator $f_{i}$. We extend it to a character $\psi^{(2)}: U_{\hbar}^{2}\left(\mathfrak{n}_{-}^{\vee}\right)=U_{\hbar}\left(\mathfrak{n}_{-}^{\vee}\right) \rtimes U\left(\mathfrak{n}_{-}^{\vee}\right) \rightarrow \mathbb{C}[\hbar]$ trivial on the second factor (its restriction to $1 \otimes U_{\hbar}\left(\mathfrak{n}_{-}^{\vee}\right)$ equals $\left.-\psi\right)$.

Definition 3.3. For $M \in \mathcal{H}_{\hbar}$ we set $\varkappa_{\hbar}(M):=\left(M \stackrel{L}{\otimes_{1 \otimes U_{\hbar}\left(\mathfrak{n}_{-}^{\vee}\right)}}(-\psi)\right)^{N_{-}^{\vee}}$ (KostantWhittaker reduction). It is equipped with an action of the Harish-Chandra center $Z\left(U_{\hbar}\right) \otimes_{\mathbb{C}[\hbar]} Z\left(U_{\hbar}\right)=\mathbb{C}\left[\mathfrak{t} / W \times \mathfrak{t} / W \times \mathbb{A}^{1}\right]$. Furthermore, $\varkappa_{\hbar}(M)$ is graded by the action of the element $h$ from a principal $\mathfrak{s l}_{2}=\langle e, h, f\rangle$-triple whose e corresponds to $\psi$ under the Killing form. All in all, $\varkappa_{\hbar}(M)$ is a $\mathbb{C}^{\times}$-equivariant coherent sheaf on $\mathfrak{t} / W \times \mathfrak{t} / W \times \mathbb{A}^{1}$ (with respect to the natural $\mathbb{C}^{\times}$-action on $\mathfrak{t} / W$ ).

3.3.1. Example: differential operators and quantum Toda lattice. The ring of $\hbar$ differential operators on $G^{\vee}, \mathcal{D}_{\hbar}\left(G^{\vee}\right)=U_{\hbar} \ltimes \mathbb{C}\left[G^{\vee}\right]$ is an object of the Indcompletion Ind $\mathcal{H C}_{\hbar}$. It carries one more commuting structure of a HarishChandra bimodule (where $U_{\hbar}$ acts by the right-invariant $\hbar$-differential operators). We define $\mathcal{K}:=\varkappa_{\hbar}\left(\mathcal{D}_{\hbar}\left(G^{\vee}\right)\right)$, an algebra in the category Ind $\mathcal{H}_{\hbar}$. It corepresents the functor $\operatorname{Hom}(M, \mathcal{K})=\varkappa_{\hbar}(D M)$ where $D M=\operatorname{Hom}_{U_{\hbar}}\left(M, U_{\hbar}\right)$ is a duality on $\mathcal{H C}_{\hbar}$. Here $\operatorname{Hom}_{U_{\hbar}}$ is taken with respect to the right action of $U_{\hbar}$, while the left actions of $U_{\hbar}$ on $M$ and on itself are used to construct the left and right actions of $U_{\hbar}$ on $\operatorname{Hom}_{U_{\hbar}}\left(M, U_{\hbar}\right)$. Finally, we define an associative algebra $\mathcal{T}_{\hbar}:=\varkappa_{\hbar}(\mathcal{K})=\varkappa_{\hbar}^{\text {right }} \varkappa_{\hbar}^{\text {left }} \mathcal{D}_{\hbar}\left(G^{\vee}\right)$, the quantum open Toda lattice. 
3.4. Deformation to the normal cone. A well known construction associates to a closed subvariety $Z \subset X$ the deformation to the normal cone $\mathcal{N}_{Z} X$ projecting to $X \times \mathbb{A}^{1}$; all the nonzero fibers are isomorphic to $X$, while the zero fiber is isomorphic to the normal cone $C_{Z} X$. Recall that $\mathcal{N}_{Z} X$ is defined as the relative spectrum of the subsheaf of algebras $\mathcal{O}_{X}\left[\hbar^{ \pm 1}\right]$, generated over $\mathcal{O}_{X \times \mathbb{A}^{1}}$ by the elements of the following type: $f \hbar^{-1}$, where $f \in \mathcal{O}_{X},\left.f\right|_{Z}=0$.

Now if $M$ is a Harish-Chandra bimodule free over $\mathbb{C}[\hbar]$, then the action of $\mathbb{C}\left[\mathfrak{t} / W \times \mathfrak{t} / W \times \mathbb{A}^{1}\right]$ on $\varkappa_{\hbar}(M)$ extends uniquely to the action of the ring of functions $\mathbb{C}\left[\mathcal{N}_{\Delta}(\mathfrak{t} / W \times \mathfrak{t} / W)\right]$ on the deformation to the normal cone of diagonal, since for $z \in Z U_{\hbar}, m \in M$, the difference of the left and right actions $z^{(1)} m-$ $z^{(2)} m$ is divisible by $\hbar$. So we will consider $\varkappa_{\hbar}(M)$ as a $\mathbb{C}^{\times}$-equivariant sheaf on $\mathcal{N}_{\Delta}(\mathfrak{t} / W \times \mathfrak{t} / W)$. Note that $\mathbb{C}\left[\mathcal{N}_{\Delta}(\mathfrak{t} / W \times \mathfrak{t} / W)\right] / \hbar=\mathbb{C}\left[C_{\Delta}(\mathfrak{t} / W \times \mathfrak{t} / W)\right]=$ $\mathbb{C}\left[T_{\mathfrak{t} / W}\right]=\mathbb{C}\left[T_{\Sigma}\right]$. Here $\Sigma \subset\left(\mathfrak{g}^{\vee}\right)^{*}$ is the Kostant slice (we identify $\mathfrak{g}^{\vee}$ and $\left(\mathfrak{g}^{\vee}\right)^{*}$ by the Killing form). Recall the universal centralizer $\mathfrak{Z}_{\mathfrak{g}^{\vee}}^{\mathfrak{g}^{\vee}}=\left\{\left(x \in \mathfrak{g}^{\vee}, \xi \in \Sigma\right)\right.$ : $\left.\operatorname{ad}_{x} \xi=0\right\}$.

Lemma 3.5. Under the identification of $\mathfrak{g}^{\vee}$ and $\left(\mathfrak{g}^{\vee}\right)^{*}$, the universal centralizer $\mathfrak{Z}_{\mathfrak{g}^{\vee}}^{\mathfrak{g}^{\vee}}$ is canonically isomorphic to the cotangent bundle $T^{*} \Sigma$.

Proof. The open subset of regular elements $\left(\mathfrak{g}^{\vee}\right)_{\text {reg }}^{*} \subset\left(\mathfrak{g}^{\vee}\right)^{*}$ carries the centralizer sheaf $\mathfrak{z} \subset \mathfrak{g}^{\vee} \otimes \mathcal{O}$ of abelian Lie algebras. We have $\mathfrak{z}=\operatorname{pr}^{*} T^{*} \Sigma$ where pr: $\left(\mathfrak{g}^{\vee}\right)_{\text {reg }}^{*} \rightarrow$ $\left(\mathfrak{g}^{\vee}\right)_{\text {reg }}^{*} / \operatorname{Ad}_{G^{\vee}}=\mathfrak{t} / W=\Sigma$ is the evident projection. Indeed, the fiber of $\operatorname{pr}^{*} T^{*} \Sigma$ at a point $\eta \in\left(\mathfrak{g}^{\vee}\right)^{*}$ is dual to the cokernel of the map $a_{\eta}: \mathfrak{g}^{\vee} \rightarrow\left(\mathfrak{g}^{\vee}\right)^{*}, x \mapsto \operatorname{ad}_{x} \eta$. In other words, this fiber is isomorphic to the kernel of the dual map which happens to coincide with $a_{\eta}$. The latter kernel is by definition nothing but the fiber $\mathfrak{z}_{\eta}$.

Lemma 3.6. For $V \in \operatorname{Rep}\left(G^{\vee}\right)$, the $\mathbb{C}\left[T_{\Sigma}\right]$-module $\left.\varkappa_{\hbar}(\operatorname{Fr}(V))\right|_{\hbar=0}$ is isomorphic to $\mathbb{C}[\Sigma] \otimes V$ as a $\mathfrak{Z}_{\mathfrak{g}^{\mathfrak{v}}}^{\mathfrak{g}^{\vee}}$-module.

Proof. Let Poly $\left(\left(\mathfrak{g}^{\vee}\right)^{*}, \mathfrak{g}^{\vee}\right)^{G^{\vee}}$ be the space of $G^{\vee}$-invariant polynomial morphisms. It is a vector bundle over Spec $\mathbb{C}\left[\left(\mathfrak{g}^{\vee}\right)^{*}\right]^{G^{\vee}}=\Sigma$. If $P \in \mathbb{C}\left[\left(\mathfrak{g}^{\vee}\right)^{*}\right]^{G^{\vee}}$, then the differential $d P$ is a section of this bundle. If $z \in Z U\left(\mathfrak{g}^{\vee}\right)=\mathbb{C}\left[\left(\mathfrak{g}^{\vee}\right)^{*}\right]^{G^{\vee}}$, we denote $d z$ by $\sigma_{z}$. If $\left\{z_{i}\right\}$ is a set of generators of $Z U\left(\mathfrak{g}^{\vee}\right)$, then $\left\{\sigma_{z_{i}}\right\}$ forms a basis of $\mathfrak{Z}_{\mathfrak{g}^{\vee}}^{\mathfrak{g}^{\vee}}$ as a vector bundle over $\Sigma$, and hence identifies it with $T^{*} \Sigma$. Let $z^{(1)}, z^{(2)}$ stand for the left and right actions of $z$ on $\operatorname{Fr}(V)$. We have to check that the action of $\left.\frac{z^{(1)}-z^{(2)}}{\hbar}\right|_{\hbar=0}$ on $\left.\left(U_{\hbar} \otimes V\right)\right|_{\hbar=0}=\mathbb{C}\left[\left(\mathfrak{g}^{\vee}\right)^{*}\right] \otimes V$ coincides with the action of $\sigma_{z} \in \mathbb{C}\left[\left(\mathfrak{g}^{\vee}\right)^{*}\right] \otimes \mathfrak{g}^{\vee}$. But if $v \in V, z=\sum_{\underline{i}} c_{\underline{\underline{y}}} x_{i_{1}} \cdots x_{i_{r}}\left(x_{i_{p}} \in \mathfrak{g}^{\vee}\right)$, and $\tilde{y} \in U_{\hbar}$ is a lift of $y \in \mathbb{C}\left[\left(\mathfrak{g}^{\vee}\right)^{*}\right]=\left.U_{\hbar}\right|_{\hbar=0}$, then $\left.\frac{z(\tilde{y} \otimes v)-(\tilde{y} \otimes v) z}{\hbar}\right|_{\hbar=0}=\sum_{i_{p} \in \underline{i}} c_{\underline{i}} x_{i_{1}} \cdots \hat{x}_{i_{p}} \cdots x_{i_{r}} y \otimes$ $x_{i_{p}}(v)=\sigma_{z}(y \otimes v)$.

3.7. Quasiclassical limit of the Kostant-Whittaker reduction. We define a functor $\varkappa$ : $\operatorname{Coh}^{G^{\vee} \times \mathbb{C}^{\times}}\left(\mathfrak{g}^{\vee}\right)^{*} \rightarrow \operatorname{Coh}^{\mathbb{C}^{\times}}(T \Sigma)$ as follows. For a $G^{\vee}$-equivariant 
coherent sheaf $\mathcal{F}$ on $\left(\mathfrak{g}^{\vee}\right)^{*}$, the restriction $\left.\mathcal{F}\right|_{\left(\mathfrak{g}^{\vee}\right)_{\text {reg }}^{*}}$ is equipped with an action of the centralizer sheaf $\mathfrak{z}$. Hence, this restriction gives rise to a coherent sheaf on $\operatorname{pr}^{*} T \Sigma$. Restricting it to the Kostant slice $\Sigma$, we obtain a coherent sheaf $\bar{\varkappa} \mathcal{F}$ on $T \Sigma$. Equivalently, the latter sheaf can be described as $\left(\left.\mathcal{F}\right|_{\Upsilon}\right)^{N_{-}}$, where $\Upsilon=e+\mathfrak{b}_{-}^{\vee} \subset \mathfrak{g}^{\vee} \simeq\left(\mathfrak{g}^{\vee}\right)^{*}$. This construction is $\mathbb{C}^{\times}$-equivariant, and gives rise to the desired functor $\varkappa$.

We define $\operatorname{Coh}_{\mathrm{fr}}^{G^{\vee} \times \mathbb{C}^{\times}}\left(\mathfrak{g}^{\vee}\right)^{*} \subset \operatorname{Coh}^{G^{\vee} \times \mathbb{C}^{\times}}\left(\mathfrak{g}^{\vee}\right)^{*}$ as the full subcategory formed by the sheaves $V \otimes \mathcal{O}_{\left(\mathfrak{g}^{\vee}\right) *}$ for $V \in \operatorname{Rep}\left(G^{\vee}\right)$.

Lemma 3.8. (a) The functors $\varkappa, \varkappa_{\hbar}$ are exact;

(b) $\left.\varkappa\right|_{\operatorname{Coh}_{\mathrm{fr}}^{G \vee} \times \mathbb{C}^{\times}\left(\mathfrak{g}^{\vee}\right)^{*}},\left.\varkappa_{\hbar}\right|_{\mathcal{H}_{\hbar}^{\mathrm{fr}}}$ are fully faithful.

Proof. The statements about $\varkappa_{\hbar}$ follow from the ones for $\varkappa$ by the graded Nakayama Lemma. To prove (a), note that the functor $\left.\mathcal{F} \mapsto \mathcal{F}\right|_{\Upsilon}, \operatorname{Coh}^{G^{\vee}}\left(\mathfrak{g}^{\vee}\right)^{*} \rightarrow \operatorname{Coh}^{N_{-}}(\Upsilon)$ is exact. Moreover, $N_{-}^{\vee}$ acts freely on $\Upsilon$, and $\Upsilon / N_{-}^{\vee}=\Sigma$. It follows that the functor $\mathcal{G} \mapsto \mathcal{G}^{N_{-}}$is exact on $\operatorname{Coh}^{N_{-}}(\Upsilon)$. Now (b) follows since the codimension in $\left(\mathfrak{g}^{\vee}\right)^{*}$ of the complement $\left(\mathfrak{g}^{\vee}\right)^{*} \backslash\left(\mathfrak{g}^{\vee}\right)_{\text {reg }}^{*}$ is at least 2 , and the centralizer of a general regular element is connected.

3.9. Equivariant cohomology of the affine Grassmannian. Now we turn to the topological machinery. We have an evident homo-

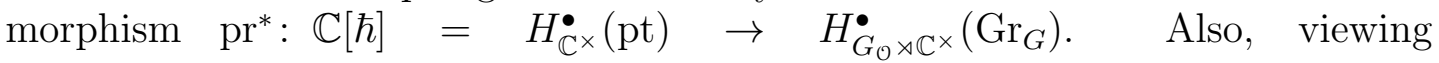
$H_{G_{\mathcal{O}} \rtimes \mathbb{C}^{\times}}^{\bullet}\left(\mathrm{Gr}_{G}\right)$ as $H_{\mathbb{C}^{\times}}^{\bullet}\left(G_{\mathcal{O}} \backslash G_{\mathcal{K}} / G_{\mathcal{O}}\right)$, we obtain two homomorphisms $\mathrm{pr}_{1}^{*}, \mathrm{pr}_{2}^{*}: \mathbb{C}[\Sigma]=\mathbb{C}[\mathfrak{t} / W]=H_{G_{\mathcal{O}}}^{\bullet}(\mathrm{pt}) \rightrightarrows H_{G_{\mathcal{O}} \rtimes \mathbb{C}^{\times}}\left(\mathrm{Gr}_{G}\right)$. Let us assume for simplicity that $G$ is simply connected. Recall the deformation to the normal cone of diagonal in $\mathfrak{t} / W \times \mathfrak{t} / W$, see Section 3.4.

Proposition 3.10. There is a natural isomorphism $\alpha: \mathbb{C}\left[\mathcal{N}_{\Delta}(\mathfrak{t} / W \times\right.$ $\mathfrak{t} / W)] \stackrel{\sim}{\longrightarrow} H_{G_{\mathcal{O}} \rtimes \mathbb{C}^{\times}}\left(\mathrm{Gr}_{G}\right)$ compatible with the above $\mathrm{pr}_{1}^{*}, \mathrm{pr}_{2}^{*}, \mathrm{pr}^{*}$.

Proof. Since $\left.H_{G_{\mathcal{O}} \rtimes \mathbb{C}^{\times}}\left(\mathrm{Gr}_{G}\right)\right|_{\hbar=0}=H_{G_{\mathcal{O}}}^{\bullet}\left(\mathrm{Gr}_{G}\right)$, we see that $\left.\operatorname{pr}_{1}^{*}\right|_{\hbar=0}=\left.\operatorname{pr}_{2}^{*}\right|_{\hbar=0}$. It follows that $\left(\mathrm{pr}_{1}^{*}, \mathrm{pr}_{2}^{*}, \mathrm{pr}^{*}\right): \mathbb{C}\left[\mathfrak{t} / W \times \mathfrak{t} / W \times \mathbb{A}^{1}\right] \rightarrow H_{G_{\mathcal{O}} \rtimes \mathbb{C}^{\times}}^{\bullet}\left(\mathrm{Gr}_{G}\right)$ factors as a composition $\mathbb{C}\left[\mathfrak{t} / W \times \mathfrak{t} / W \times \mathbb{A}^{1}\right] \rightarrow \mathbb{C}\left[\mathcal{N}_{\Delta}(\mathfrak{t} / W \times \mathfrak{t} / W)\right] \stackrel{\alpha}{\rightarrow} H_{G_{\mathcal{O}} \rtimes \mathbb{C}^{\times}}\left(\mathrm{Gr}_{G}\right)$ for a uniquely defined homomorphism $\alpha$. Let us check that $\alpha$ is injective. Indeed, $\alpha_{\text {loc }}: \mathbb{C}\left[\mathcal{N}_{\Delta}(\mathfrak{t} / W \times \mathfrak{t} / W)\right] \otimes_{\mathbb{C}\left[\mathfrak{t} / W \times \mathbb{A}^{1}\right]} \operatorname{Frac}\left(\mathbb{C}\left[\mathfrak{t} \times \mathbb{A}^{1}\right]\right) \rightarrow H_{G_{\mathcal{O}} \times \mathbb{C} \times}\left(\operatorname{Gr}_{G}\right) \otimes_{\mathbb{C}\left[\mathfrak{t} / W \times \mathbb{A}^{1}\right]}$ $\operatorname{Frac}\left(\mathbb{C}\left[\mathfrak{t} \times \mathbb{A}^{1}\right]\right)=H_{T \times \mathbb{C}^{\times}}^{\bullet}\left(\operatorname{Gr}_{G}\right) \otimes_{\mathbb{C}\left[\mathfrak{t} \times \mathbb{A}^{1}\right]} \operatorname{Frac}\left(\mathbb{C}\left[\mathfrak{t} \times \mathbb{A}^{1}\right]\right) \hookrightarrow \lim _{\leftarrow} H_{T \times \mathbb{C}^{\times}}^{\bullet}\left(\overline{\operatorname{Gr}}_{G}^{\lambda}\right) \otimes_{\mathbb{C}\left[\mathfrak{t} \times \mathbb{A}^{1}\right]}$ $\operatorname{Frac}\left(\mathbb{C}\left[\mathfrak{t} \times \mathbb{A}^{1}\right]\right)=\prod_{\lambda \in X_{*}(T)} \operatorname{Frac}\left(\mathbb{C}\left[\mathfrak{t} \times \mathbb{A}^{1}\right]\right)$ associates to a function its restriction to the union of graphs $\Gamma_{\lambda}:=\left\{\left(x_{1}, x_{2}, c\right): x_{1}=x_{2}+c \lambda\right\} \subset \mathfrak{t} \times \mathfrak{t} \times \mathbb{A}^{1}$. More precisely, for a $T$-fixed point $\lambda \in \mathrm{Gr}_{G}$, the $\mathbb{C}\left[\mathfrak{t} \times(\mathfrak{t} / W) \times \mathbb{A}^{1}\right]$-module $H_{T \times \mathbb{C} \times}^{\bullet}(\lambda)$ is canonically isomorphic to $(\mathrm{Id}, \pi, \mathrm{Id})_{*} \mathcal{O}_{\Gamma_{\lambda}}$, where $\pi$ stands for the projection $\mathfrak{t} \rightarrow \mathfrak{t} / W$. Indeed, let $p: \mathcal{F} \ell_{G} \rightarrow \operatorname{Gr}_{G}$ be the projection from the affine flag 
variety $\mathcal{F} \ell_{G}=G_{\mathcal{K}} /$ Iw of $G$, and let $\tilde{\lambda} \in \mathcal{F} \ell_{G}$ be the $T$-fixed point in $p^{-1}(\lambda)$ corresponding to the coweight $\lambda \in X_{*}(T) \subset W_{\text {aff }}$. Viewing $H_{T \times \mathbb{C}^{\times}}^{\bullet}\left(\mathcal{F} \ell_{G}\right)$ as $H_{\mathbb{C}^{\times}}^{\bullet}\left(\mathrm{Iw} \backslash G_{\mathcal{K}} / \mathrm{Iw}\right)$, we identify $H_{T \times \mathbb{C}^{\times}}^{\bullet}(\tilde{\lambda})$ with a $\mathbb{C}\left[\mathfrak{t} \times \mathfrak{t} \times \mathbb{A}^{1}\right]$-module $M$ such that $(\mathrm{Id}, \pi, \mathrm{Id})_{*} M=H_{T \times \mathbb{C}^{\times}}(\lambda)$. The preimage $T_{\lambda}$ of $\tilde{\lambda}$ in $G_{\mathcal{K}}$ is homotopy equivalent to the torus $T$, and the action of $T \times T \times \mathbb{C}^{\times}$on $T_{\lambda}$ is homotopy equivalent to $\left(t_{1}, t_{2}, c\right) \cdot t=t_{1} t t_{2}^{-1} \lambda(c)$. We conclude that $H_{T \times \mathbb{C}^{\times}}^{\bullet}(\tilde{\lambda})=H_{\mathbb{C}^{\times}}^{\bullet}\left(T \backslash T_{\lambda} / T\right)=\mathbb{C}\left[\Gamma_{\lambda}\right]$.

Finally, the union $\bigcup_{\lambda \in X_{*}(T)} \Gamma_{\lambda}$ is Zariski dense in $\mathfrak{t} \times \mathfrak{t} \times \mathbb{A}^{1}$. Hence $\alpha_{\text {loc }}$ is injective, and $\alpha$ is injective as well.

To finish the proof it suffices to compare the graded dimensions of the LHS and the RHS (the grading in the LHS arises from the natural action of $\mathbb{C}^{\times}$on $\mathfrak{t}$ and $\left.\mathbb{A}^{1}\right)$. Now $\operatorname{dim}_{\mathrm{gr}}\left(H_{G_{\mathcal{O}} \rtimes \mathbb{C}^{\times}}\left(\operatorname{Gr}_{G}\right)\right)=\operatorname{dim}_{\mathrm{gr}}\left(H_{G_{\mathcal{O}}}^{\bullet}(\mathrm{pt}) \otimes H_{\mathbb{C}^{\times}}^{\bullet}(\mathrm{pt}) \otimes H^{\bullet}\left(\mathrm{Gr}_{G}\right)\right)=$ $\operatorname{dim}_{\mathrm{gr}} \mathbb{C}\left[x_{1}, \ldots, x_{r}, y_{1}, \ldots, y_{r}, \hbar\right]$ where $\operatorname{deg} \hbar=2, \operatorname{deg} x_{i}=\operatorname{deg} y_{i}=2\left(m_{i}+1\right)$, and $m_{1}, \ldots, m_{r}$ are the exponents of $G$ (so that $m_{i}+1$ are the degrees of generators of $W$-invariant polynomials on $\mathfrak{t})$.

Furthermore, $\mathfrak{t} / W=\Sigma$, and $\mathcal{N}_{\Delta}(\Sigma \times \Sigma) \simeq \Sigma \times \Sigma \times \mathbb{A}^{1}$ (an affine space). Indeed, for affine spaces $V, V^{\prime}$ we have an isomorphism $\beta: V \times V^{\prime} \times \mathbb{A}^{1} \stackrel{\sim}{\longrightarrow} \mathcal{N}_{V}\left(V \times V^{\prime}\right)$; namely, $\gamma: V \times V^{\prime} \times \mathbb{A}^{1} \rightarrow V \times V^{\prime} \times \mathbb{A}^{1},\left(v, v^{\prime}, c\right) \mapsto\left(v, c v^{\prime}, c\right)$ factors through $V \times V^{\prime} \times \mathbb{A}^{1} \stackrel{\beta}{\rightarrow} \mathcal{N}_{V \times V^{\prime}} V \rightarrow V \times V^{\prime} \times \mathbb{A}^{1}$. We conclude that $\operatorname{dim}_{\mathrm{gr}}(\mathrm{LHS})=$ $\operatorname{dim}_{\mathrm{gr}}(\mathrm{RHS})$. This completes the proof.

In view of Proposition 3.10, we can view $H_{G_{\mathcal{O}} \rtimes \mathbb{C}^{\times}}\left(\mathrm{Gr}_{G},-\right)$ as a functor from the full subcategory $\mathcal{J C}_{G_{\mathcal{O}} \rtimes \mathbb{C} \times} \subset D_{G_{\mathcal{O}} \rtimes \mathbb{C} \times}^{b}\left(\mathrm{Gr}_{G}\right)$ formed by the semisimple complexes, to $\operatorname{Coh}^{\mathbb{C}^{\times}}\left(\mathcal{N}_{\Delta}(\mathfrak{t} / W \times \mathfrak{t} / W)\right)$. This functor is fully faithful according to [Gi2]. For $V \in \operatorname{Rep}\left(G^{\vee}\right)$, one can identify $H_{G_{\mathcal{O}} \rtimes \mathbb{C}^{\times}}\left(\operatorname{Gr}_{G}, S(V)\right)$ with $\varkappa_{\hbar}(\operatorname{Fr}(V))$; moreover, one can make this identification compatible with the tensor structures on $\operatorname{Rep}\left(G^{\vee}\right)$ and $\operatorname{Perv}_{G_{\mathcal{O}}}\left(\mathrm{Gr}_{G}\right)[\mathrm{BeFi}]$ :

Theorem 3.11. The geometric Satake equivalence $\mathbb{S}: \operatorname{Rep}\left(G^{\vee}\right) \stackrel{\sim}{\longrightarrow} \operatorname{Perv}_{G_{\mathcal{O}}}\left(\operatorname{Gr}_{G}\right)$ extends to a tensor equivalence $\mathbb{S}_{\hbar}: \mathcal{H C}_{\hbar}^{\mathrm{fr}} \stackrel{\sim}{\longrightarrow} \mathcal{J}_{G_{\mathcal{O}} \rtimes \mathbb{C} \times}$ such that $\varkappa_{\hbar}=H_{G_{\mathcal{O}} \rtimes \mathbb{C}^{\times}}\left(\mathrm{Gr}_{G},-\right) \circ \mathbb{S}_{\hbar}$. There is also a quasiclassical version $\mathbb{S}_{q c}: \operatorname{Coh}_{\mathrm{fr}}^{G^{\vee} \times \mathbb{C}^{\times}}\left(\mathfrak{g}^{\vee}\right)^{*} \stackrel{\sim}{\longrightarrow} \mathcal{J C}_{G_{\mathcal{O}}}$ such that $\varkappa=H_{G_{\mathcal{O}}}^{\bullet}\left(\mathrm{Gr}_{G},-\right) \circ \mathbb{S}_{q c}$.

Now using the formality of RHom-algebras in our categories, one can deduce the desired derived geometric Satake equivalence. To formulate it, we introduce a bit of notation. To a dg-algebra $A$ one can associate the triangulated category of dg-modules localized by quasi-isomorphisms, and a full triangulated subcategory $D_{\text {perf }}(A) \subset D(A)$ of perfect complexes. Given an algebraic group $H$ acting on $A$, we can consider $H$-equivariant dg-modules and localize them by quasiisomorphisms, arriving at the equivariant version $D_{\text {perf }}^{H}(A)$.

We now consider the dg-versions $\operatorname{Sym}^{[]}\left(\mathfrak{g}^{\vee}\right), U_{\hbar}^{[]}$of the graded algebras $\operatorname{Sym}\left(\mathfrak{g}^{\vee}\right), U_{\hbar}$, equipping them with the zero differential and the cohomological 
grading so that elements of $\mathfrak{g}^{\vee}$ and $\hbar$ have degree 2. The construction of the previous paragraph gives rise to the categories $D_{\text {perf }}^{G^{\vee}}\left(U_{\hbar}^{[]}\right), D_{\text {perf }}^{G^{\vee}}\left(\operatorname{Sym}^{[]}\left(\mathfrak{g}^{\vee}\right)\right)$. Their Ind-completions will be denoted by $D^{G^{\vee}}\left(U_{\hbar}^{[]}\right), D^{G^{\vee}}\left(\operatorname{Sym}^{[]}\left(\mathfrak{g}^{\vee}\right)\right)$. The Ind-completions of the equivariant derived categories $D_{G_{\mathcal{O}} \rtimes \mathbb{C}^{\times}}^{b}\left(\mathrm{Gr}_{G}\right), D_{G_{\mathcal{O}}}^{b}\left(\mathrm{Gr}_{G}\right)$ will be denoted by $D_{G_{\mathcal{O}} \rtimes \mathbb{C}^{\times}}\left(\mathrm{Gr}_{G}\right), D_{G_{\mathcal{O}}}\left(\mathrm{Gr}_{G}\right)$.

The following theorem is proved in $[\mathrm{BeFi}$.

Theorem 3.12. The equivalences of Theorem 3.11 extend to the equivalences of monoidal triangulated categories $\Psi_{\hbar}: D_{\text {perf }}^{G^{\vee}}\left(U_{\hbar}^{[]}\right) \stackrel{\sim}{\longrightarrow} D_{G_{\mathcal{O}} \rtimes \mathbb{C}^{\times}}^{b}\left(\operatorname{Gr}_{G}\right)$ and $\Psi_{q c}: D_{\text {perf }}^{G^{\vee}}\left(\operatorname{Sym}^{[]}\left(\mathfrak{g}^{\vee}\right)\right) \stackrel{\sim}{\longrightarrow} D_{G_{\mathcal{O}}}^{b}\left(\operatorname{Gr}_{G}\right)$. They induce the equivalences of their Ind-completions $\Psi_{\hbar}: D^{G^{\vee}}\left(U_{\hbar}^{[]}\right) \stackrel{\sim}{\longrightarrow} D_{G_{\mathcal{O}} \rtimes \mathbb{C} \times}\left(\operatorname{Gr}_{G}\right)$ and $\Psi_{q c}: D^{G^{\vee}}\left(\operatorname{Sym}^{[]}\left(\mathfrak{g}^{\vee}\right)\right) \stackrel{\sim}{\longrightarrow} D_{G_{\mathcal{O}}}\left(\mathrm{Gr}_{G}\right)$.

3.12.1. The dualities. We denote by $\mathfrak{C}_{G^{\vee}}$ the autoequivalence of $D^{G^{\vee}}\left(U_{\hbar}^{[]}\right)$induced by the canonical outer automorphism of $G^{\vee}$ interchanging conjugacy classes of $g$ and $g^{-1}$ (the Chevalley involution). We also denote by $\mathcal{C}_{G}$ the autoequivalence of $D_{G_{\mathcal{O}} \rtimes \mathbb{C}^{\times}}\left(\operatorname{Gr}_{G}\right)$ induced by $g \mapsto g^{-1}, G((z)) \rightarrow G((z))$. Then the Verdier duality $\mathbb{D}: D_{G_{\mathcal{O}} \rtimes \mathbb{C}^{\times}}\left(\mathrm{Gr}_{G}\right) \rightarrow D_{G_{\mathcal{O}} \rtimes \mathbb{C}^{\times}}\left(\mathrm{Gr}_{G}\right)$ and the duality $D: D^{G^{\vee}}\left(U_{\hbar}^{[]}\right) \rightarrow D^{G^{\vee}}\left(U_{\hbar}^{[]}\right)$ introduced in Example 3.3.1 are related by $\Psi_{\hbar} \circ \mathfrak{C}_{G^{\vee}} \circ D=\mathbb{D} \circ \Psi_{\hbar}$.

3.13. The regular sheaf. Recall the setup of Example 3.3.1. We consider $\mathcal{D}_{\hbar}^{[]}\left(G^{\vee}\right)=U_{\hbar}^{[]} \ltimes \mathbb{C}\left[G^{\vee}\right] \in D^{G^{\vee}}\left(U_{\hbar}^{[]}\right)$. Its image under the equivalence of Theorem 3.12 is the regular sheaf $\mathcal{A}_{R}^{\mathbb{C}^{\times}} \in D_{G_{\mathcal{O}} \rtimes \mathbb{C}^{\times}}\left(\mathrm{Gr}_{G}\right)$ isomorphic to $\bigoplus_{\lambda \in \Lambda^{+}} \operatorname{IC}\left(\overline{\mathrm{Gr}}_{G}^{\lambda}\right) \otimes$ $\left(V^{\lambda}\right)^{*}$. The quasiclassical analogs are $D^{G^{\vee}}\left(\operatorname{Sym}^{[]}\left(\mathfrak{g}^{\vee}\right)\right) \ni \mathbb{C}\left[T^{*} G^{\vee}\right]^{[]}=\operatorname{Sym}^{[]}\left(\mathfrak{g}^{\vee}\right) \otimes$ $\mathbb{C}\left[G^{\vee}\right] \mapsto \mathcal{A}_{R} \in D_{G_{\mathcal{O}}}\left(\mathrm{Gr}_{G}\right)$. One can check that the image of $\varkappa_{\hbar} \mathcal{D}_{\hbar}^{[]}\left(G^{\vee}\right) \in$ $D^{G^{\vee}}\left(U_{\hbar}^{[]}\right)$under the equivalence of Theorem 3.12 is the dualizing sheaf $\boldsymbol{\omega}_{\mathrm{Gr}_{G}} \in$ $D_{G_{\mathcal{O}} \rtimes \mathbb{C}^{\times}}\left(\mathrm{Gr}_{G}\right)$. It follows that the convolution algebra of equivariant Borel-Moore homology $H_{\bullet}^{G_{\mathcal{O}} \rtimes \mathbb{C}^{\times}}\left(\mathrm{Gr}_{G}\right)=H_{G_{\mathcal{O}} \rtimes \mathbb{C}^{\times}}\left(\mathrm{Gr}_{G}, \boldsymbol{\omega}_{\mathrm{Gr}_{G}}\right)$ is isomorphic to quantum open Toda lattice $\mathcal{T}_{\hbar}=\varkappa_{\hbar}^{\text {right }} \varkappa_{\hbar}^{\text {left }} \mathcal{D}_{\hbar}\left(G^{\vee}\right)$ of Example 3.3.1.

Note that the regular sheaf $\mathcal{A}_{R}^{\mathbb{C}^{\times}}$is equipped with an action of $G^{\vee}$. Furthermore, the dg-algebra $\operatorname{RHom}_{D_{G_{\mathcal{O}} \rtimes \mathbb{C}^{\times}}\left(\operatorname{Gr}_{G}\right)}\left(\mathcal{A}_{R}^{\mathbb{C}^{\times}}, \mathcal{A}_{R}^{\mathbb{C}^{\times}}\right)$is formal, and we have a $G^{\vee}$-equivariant morphism of dg-algebras $U_{\hbar}^{[]} \rightarrow \operatorname{RHom}_{D_{G_{\mathcal{O}} \rtimes \mathbb{C} \times}\left(\operatorname{Gr}_{G}\right)}\left(\mathcal{A}_{R}^{\mathbb{C} \times}, \mathcal{A}_{R}^{\mathbb{C}^{\times}}\right)$ (corresponding to the right action of $U_{\hbar}^{[]}$on $\mathcal{D}_{\hbar}^{[]}\left(G^{\vee}\right)$ ). Similarly, the dg-algebra $\mathrm{RHom}_{D_{G_{\mathcal{O}}}\left(\mathrm{Gr}_{G}\right)}\left(\mathcal{A}_{R}, \mathcal{A}_{R}\right)$ is formal, and we have a $G^{\vee}$-equivariant morphism of dg-algebras $\operatorname{Sym}^{[]}\left(\mathfrak{g}^{\vee}\right) \rightarrow \operatorname{RHom}_{D_{G_{\mathcal{O}}}\left(\operatorname{Gr}_{G}\right)}\left(\mathcal{A}_{R}, \mathcal{A}_{R}\right)$ (corresponding to the right action of $\operatorname{Sym}^{[]}\left(\mathfrak{g}^{\vee}\right)$ on $\left.\mathbb{C}\left[T^{*} G^{\vee}\right]^{[]}\right)$. Hence for any $\mathcal{F} \in D_{G_{\mathcal{O}} \rtimes \mathbb{C}^{\times}}\left(\operatorname{Gr}_{G}\right)$, the complex $\operatorname{RHom}_{D_{G_{\mathcal{O}} \rtimes \mathbb{C}^{\times}}\left(\operatorname{Gr}_{G}\right)}\left(\mathcal{A}_{R}^{\mathbb{C}^{\times}}, \mathcal{F}\right)$ carries a structure of $G^{\vee}$-equivariant dg-module over $U_{\hbar}^{[]}$. 
Thus the functors $\mathrm{RHom}_{D_{G_{\mathcal{O}} \rtimes \mathbb{C}^{\times}}\left(\operatorname{Gr}_{G}\right)}\left(\mathcal{A}_{R}^{\mathbb{C}^{\times}}, \bullet\right), \operatorname{RHom}_{D_{G_{\mathcal{O}}}\left(\operatorname{Gr}_{G}\right)}\left(\mathcal{A}_{R}, \bullet\right)$ may be viewed as landing respectively into $D^{G^{\vee}}\left(U_{\hbar}^{[]}\right), D^{G^{\vee}}\left(\operatorname{Sym}^{\square}\left(\mathfrak{g}^{\vee}\right)\right)$. We will also need their versions

$$
\begin{aligned}
\Phi_{\hbar}:=\operatorname{RHom}_{D_{G_{\mathcal{O}} \rtimes \mathbb{C}^{\times}}\left(\operatorname{Gr}_{G}\right)}\left(\mathbf{1}_{\mathrm{Gr}_{G}}, \mathcal{C}_{G} \mathcal{A}_{R}^{\mathbb{C}^{\times}} \star \bullet\right) \stackrel{\sim}{\longrightarrow} \operatorname{RHom}_{D_{G_{\mathcal{O}} \rtimes \mathbb{C}^{\times}}\left(\operatorname{Gr}_{G}\right)}\left(\mathbb{D} \mathcal{A}_{R}^{\mathbb{C}^{\times}}, \bullet\right) \\
\stackrel{\sim}{\sim} \operatorname{RHom}_{D_{G_{\mathcal{O}} \rtimes \mathbb{C}^{\times}}\left(\operatorname{Gr}_{G}\right)}\left(\mathbb{C}_{G_{G}}, \mathcal{A}_{R}^{\mathbb{C}^{\times}} \otimes ! \bullet\right),
\end{aligned}
$$

and

$$
\begin{aligned}
\Phi_{q c}:=\operatorname{RHom}_{D_{G_{\mathcal{O}}}\left(\mathrm{Gr}_{G}\right)}\left(\mathbf{1}_{\mathrm{Gr}_{G}}, \mathcal{C}_{G} \mathcal{A}_{R} \star \bullet\right) & \stackrel{\sim}{\sim} \operatorname{RHom}_{D_{G_{\mathcal{O}}}\left(\mathrm{Gr}_{G}\right)}\left(\mathbb{D}_{\left.\mathcal{A}_{R}, \bullet\right)}\right. \\
& \sim \operatorname{RHom}_{D_{G_{\mathcal{O}}}\left(\mathrm{Gr}_{G}\right)}\left(\mathbb{C}_{\mathrm{Gr}_{G}}, \mathcal{A}_{R} \otimes ! \bullet\right) .
\end{aligned}
$$

The following lemma is proved in [BFN3].

Lemma 3.14. (a) The functors $\operatorname{RHom}_{D_{G_{\mathcal{O}} \rtimes \mathbb{C}^{\times}}\left(\operatorname{Gr}_{G}\right)}\left(\mathcal{A}_{R}^{\mathbb{C}^{\times}}, \bullet\right): D_{G_{\mathcal{O}} \rtimes \mathbb{C} \times}\left(\operatorname{Gr}_{G}\right) \rightarrow$ $D^{G^{\vee}}\left(U_{\hbar}^{[]}\right)$and $\operatorname{RHom}_{D_{G_{\mathcal{O}}}\left(\operatorname{Gr}_{G}\right)}\left(\mathcal{A}_{R}, \bullet\right): D_{G_{\mathcal{O}}}\left(\operatorname{Gr}_{G}\right) \rightarrow D^{G^{\vee}}\left(\operatorname{Sym}^{[]}\left(\mathfrak{g}^{\vee}\right)\right)$ are canonically isomorphic to $\Psi_{\hbar}^{-1}$ and $\Psi_{q c}^{-1}$ respectively.

(b) The functors $\Phi_{\hbar}: D_{G_{\mathcal{O}} \rtimes \mathbb{C}^{\times}}\left(\operatorname{Gr}_{G}\right) \rightarrow D^{G^{\vee}}\left(U_{\hbar}^{[]}\right)$and $\Phi_{q c}: D_{G_{\mathcal{O}}}\left(\mathrm{Gr}_{G}\right) \rightarrow$ $D^{G^{\vee}}\left(\operatorname{Sym}^{[]}\left(\mathfrak{g}^{\vee}\right)\right)$ are canonically isomorphic to $\mathfrak{C}_{G^{\vee}} \circ \Psi_{\hbar}^{-1}$ and $\mathfrak{C}_{G^{\vee}} \circ \Psi_{q c}^{-1}$ respectively.

\section{Motivation II: What do We (AS Mathematicians) Might WAnt FROM 3D N=4 SUSY QFT? (NAIVE APPROACH)}

4.1. Some generalities. In this Section we would like to introduce some language related to 3 -dimensional $\mathrm{N}=4$ super-symmetric quantum field theories. The reader should be warned from the very beginning: here we are going to use all the words from physics as a "black box". More precisely, we are not going to try to explain what such a theory really is from a mathematical point of view. Instead we are going to review the relevant "input data" (i.e. to what mathematical structures physicists usually attach such a QFT) and some of the "output data" (i.e. what mathematical structures one should get in the end. This will be largely extended in Section 7, where we partly address the question "what kind of structures these 3d N=4 SUSY QFTs really are from a mathematical point of view?"). Also it will be important for us to recall (in this Section) what one can do with these theories: i.e. we are going to discuss some operations which produce new quantum field theories out of old ones.

The reader should be warned from the very beginning about the following: both in this Section and in Section 7 we are only going to discuss algebraic aspects of the above quantum field theories (such as e.g. algebraic varieties or categories one can attach to them). In principle "true physical theory" is supposed to have some interesting analytic aspects (such as e.g. a metric on the above varieties). 
These analytic aspects will be completely ignored in these notes. Essentially, this means that we are going to study quantum field theories up to certain "algebraic equivalence" but we are not going to discuss details in these notes.

4.2. Higgs and Coulomb branch and 3d mirror symmetry. A $3 d \quad N=4$ super-symmetric quantum field theory $\mathcal{T}$ is supposed to have a well-defined moduli space of vacua. This should be some complicated (though interesting) space. This space is somewhat too complicated for our present discussion. Instead we are going to discuss some "easy" parts of this space. Namely, the above moduli space of vacua should have two special pieces called the Higgs and the Coulomb branch of the moduli space of vacua; we shall denote these by $\mathcal{M}_{H}(\mathcal{T})$ and $\mathcal{M}_{C}(\mathcal{T})$. They are supposed to be Poisson (generically symplectic) complex algebraic varieties. ${ }^{4}$ They should also be hyper-kähler in some sense, but (to the best of our knowledge) this notion is not well-defined for singular varieties, we are going to ignore the hyper-kähler structure in these notes. So, in this section we are going to think about a theory $\mathcal{T}$ in terms of $\mathcal{M}_{H}(\mathcal{T})$ and $\mathcal{M}_{C}(\mathcal{T})$. Of course, this is a very small part of what the actual "physical theory" is, but we shall see that even listing the structures that physicists expect from $\mathcal{M}_{H}(\mathcal{T})$ and $\mathcal{M}_{C}(\mathcal{T})$ will lead us to interesting constructions.

One of the operations on theories that will be important in the future is the operation of "3-dimensional mirror symmetry". Namely, physicists expect that for a theory $\mathcal{T}$ there should exist a mirror dual theory $\mathcal{T}^{*}$ such that $\mathcal{M}_{H}\left(\mathcal{T}^{*}\right)=$ $\mathcal{M}_{C}(\mathcal{T})$ and $\mathcal{M}_{C}\left(\mathcal{T}^{*}\right)=\mathcal{M}_{H}(\mathcal{T})$.

4.3. More operations on theories. In what follows we shall use the following notation: for a symplectic variety $X$ with a Hamiltonian $G$-action we shall denote by $X / / / G$ the Hamiltonian reduction of $X$ with respect to $G$.

Then the following operations on theories are expected to make sense (in the next subsection we shall start considering examples) :

1) If $\mathcal{T}_{1}, \cdots, \mathcal{T}_{n}$ are some theories then one can form their product $\mathcal{T}_{1} \times \cdots \times \mathcal{T}_{n}$. We have

$$
\mathcal{M}_{H}\left(\mathcal{T}_{1} \times \cdots \times \mathcal{T}_{n}\right)=\mathcal{M}_{H}\left(\mathcal{T}_{1}\right) \times \cdots \times \mathcal{M}_{H}\left(\mathcal{T}_{n}\right)
$$

and

$$
\mathcal{M}_{C}\left(\mathcal{T}_{1} \times \cdots \times \mathcal{T}_{n}\right)=\mathcal{M}_{C}\left(\mathcal{T}_{1}\right) \times \cdots \times \mathcal{M}_{C}\left(\mathcal{T}_{n}\right)
$$

2) Let $\mathcal{T}$ be a theory and let $G$ be a complex reductive group. Then there is a notion of $G$ acting on $\mathcal{T}$. Physicists say in this case that $G$ maps to the flavor symmetry group of $\mathcal{T}$, or that we are given a theory $\mathcal{T}$ with flavor symmetry $G$.

\footnotetext{
${ }^{4}$ In fact, this is already a simplification: non-algebraic holomorphic symplectic manifolds should also arise in this way, but we are not going to discuss such theories.
} 
Assume that we are given a theory $\mathcal{T}$ with flavor symmetry $G$. Then there is a new theory $\mathcal{T} / G$ obtained by "gauging" $G$. The origin of the notation is explained in 3).

3) We have $\mathcal{M}_{H}(\mathcal{T} / G)=\mathcal{M}_{H}(\mathcal{T}) / / G$. Of course, the notion of Hamiltonian reduction can be understood in several different ways, so we need to talk a little about what we mean by $/ / / G$ here. Recall that the Hamiltonian reduction is defined as follows. Let $\mathcal{X}$ be any Poisson variety endowed with a Hamiltonian action of $G$. Then we have the moment map $\mu: \mathcal{X} \rightarrow \mathfrak{g}^{*}$. Then we are supposed to have $X / / / G=\left(\mu^{-1}(0)\right) / G$. Here there are two delicate points. First, the map $\mu$ might not be flat, so honestly we must take $\mu^{-1}(0)$ in the dg-sense. Second, we must specify what we mean by quotient by $G$. In these notes we shall mostly deal with examples when $\mathcal{X}$ is affine and we shall be primarily interested in the algebra of functions on $X / / / G$. For these purposes it is enough to work with the so called "categorical quotient", i.e. we set

$$
\mathbb{C}[X / / G]=\left(\mathbb{C}\left[\mu^{-1}(0)\right]\right)^{G} .
$$

Note that according to our conventions this might be a dg-algebra.

4) Given $\mathcal{T}$ and $G$ as above (assuming that $G$ is connected and reductive) one can construct a ring object $\mathcal{A}_{\mathcal{T}}$ in $D_{G_{\mathcal{O}}}\left(\mathrm{Gr}_{G}\right)$ (sometimes we shall denote it by $\mathcal{A}_{\mathcal{T}, G}$ when we need to stress the dependence on $\left.G\right)$. The !-stalk of $\mathcal{A}_{\mathcal{T}}$ at the unit point of $\operatorname{Gr}_{G}$ is $\mathbb{C}\left[\mathcal{M}_{C}(\mathcal{T})\right]$ and $H_{G_{\mathcal{O}}}^{*}\left(\mathcal{A}_{\mathcal{T}}\right)=\mathbb{C}\left[\mathcal{M}_{C}(\mathcal{T} / G)\right]$. In fact, the object $\mathcal{A}_{\mathcal{T}}$ should also have a Poisson structure (which will induce a Poisson structure on $\mathbb{C}\left[\mathcal{M}_{C}(\mathcal{T})\right]$ and on $\mathcal{M}_{C}(\mathcal{T} / G)$ ) but we are going to ignore this issue for now.

Let us as before denote by $i$ the emebedding of the the point 1 in $\mathrm{Gr}_{G}$. Then $i^{!} \mathcal{A}_{\mathcal{T}}$ can be regarded as a ring object of the equivariant derived category $D_{G}(\mathrm{pt})$. Its equivariant cohomology $H_{G}^{*}\left(\mathrm{pt}, i^{!} \mathcal{A}_{\mathcal{T}}\right)$ is a graded algebra over $H_{G}^{*}(\mathrm{pt}, \mathbb{C})=$ $\mathbb{C}[\mathfrak{g}]^{G}=\mathbb{C}[\mathfrak{t}]^{W}$ whose (derived) fiber over 0 is equal to $\mathbb{C}\left[\mathcal{M}_{C}\right]$. Thus flavor symmetry $G$ is supposed to define a (Poisson) deformation of $\mathcal{M}_{C}$ over the base $\mathfrak{t} / W$. In particular, by base change we should have a Poisson deformation of $\mathcal{M}_{C}$ over $\boldsymbol{t}$.

5) Let $H$ be a subgroup of $G$. Then $\mathcal{A}_{\mathcal{T}, H}$ is equal to the !-restriction of $\mathcal{A}_{\mathcal{T}, G}$ to $\mathrm{Gr}_{H}$.

6) For a reductive group $G$ there is a theory $\mathcal{T}[G]$ such that

a) $\mathcal{T}[G]$ has flavor symmetry $G$.

b) $\mathcal{M}_{H}(\mathcal{T}[G])=\mathcal{N}_{\mathfrak{g}}, \mathcal{M}_{C}(\mathcal{T}[G])=\mathcal{N}_{\mathfrak{g} \vee}$; here $\mathfrak{g}=\operatorname{Lie}(G)$ and $\mathcal{N}_{\mathfrak{g}}$ is its nilpotent cone.

c) $\mathcal{A}_{\mathcal{T}\left[G^{\vee}\right]}=\mathcal{A}_{R}$ (our "regular representation" sheaf on $\mathrm{Gr}_{G}$ ).

d) $\mathcal{T}[G]^{*}=\mathcal{T}\left[G^{\vee}\right]$.

7) For a theory $\mathcal{T}$ with flavor symmetry $G$ there should exist another (S-dual) theory $\mathcal{T}^{\vee}$ with flavor symmetry $G^{\vee}$ (it is defined via S-duality for 4-dimensional 
$\mathrm{N}=4$ super-symmetric gauge theory). Gaiotto and Witten [GW] claim that

$$
\mathcal{T}^{\vee}=((\mathcal{T} \times \mathcal{T}[G]) / G)^{*}
$$

(here the gauging is taken with respect to diagonal copy of $G$ ).

In particular, the RHS of (2) has an action of $G^{\vee}$ (which a priori is absolutely non-obvious). Here is an example: take $\mathcal{T}$ to be the trivial theory with trivial $G$-action (in this case $\mathcal{M}_{H}=\mathcal{M}_{C}=$ pt, but the structure is still somewhat nontrivial as we remember the group $G$ ). Then $\mathcal{T} \times \mathcal{T}[G]=\mathcal{T}[G]$. Now $\mathcal{T}[G] / G$ is the theory whose (naive - i.e. not dg) Higgs branch is pt and whose Coulomb branch is isomorphic to $G^{\vee} \times \mathfrak{t} / W=\operatorname{Spec}\left(H_{G_{\mathcal{O}}}^{*}\left(\operatorname{Gr}_{G}, \mathcal{A}_{R}\right)\right)$. Since the mirror duality interchanges $\mathcal{M}_{H}$ and $\mathcal{M}_{C}$ we see that $\mathcal{M}_{H}\left(\mathcal{T}^{\vee}\right)$ has an action of $G^{\vee}$.

More generally, it follows that

$$
\mathbb{C}\left[\mathcal{M}_{H}\left(\mathcal{T}^{\vee}\right)\right]=H_{G_{\mathcal{O}}}^{*}\left(\mathrm{Gr}_{G}, \mathcal{A}_{\mathcal{T}} \stackrel{!}{\otimes} \mathcal{A}_{R}\right)
$$

(this follows from 5). In particular, it has a natural action of $G^{\vee}$.

Let us now pass to examples.

4.4. Basic example. This is in some sense the most basic example. Let $\mathbf{M}$ be a connected symplectic algebraic variety over $\mathbb{C}$. Then to $\mathbf{M}$ there should correspond a theory $T(\mathbf{M})$ for which $\mathcal{M}_{H}=\mathbf{M}$ and $\mathcal{M}_{C}=$ pt. In fact, this is true only if dg-structures are disregarded. However, in these notes we shall mostly care about the case when $\mathbf{M}$ is just a symplectic vector space and in this case it should be true as stated (cf. Subsection 7.14 for more details).

4.5. Gauge theories. Let $G$ be a reductive group. Then an action of $G$ on $T(\mathbf{M})$ should be the same as a Hamiltonian action of $G$ on $\mathbf{M}{ }^{5}$ Then we can form the theory $T(\mathbf{M}) / G$.

Assume that $\mathbf{M}$ is actually a symplectic vector space and that the action of $G$ on $\mathbf{M}$ is linear. Then the theory $T(\mathbf{M}) / G$ is called gauge theory with matter $\mathbf{M}$. In the case when $\mathbf{M}=\mathbf{N} \oplus \mathbf{N}^{*}=T^{*} \mathbf{N}$ for some repesentation $\mathbf{N}$ of $G$, the Coulomb branch of these theories together with the corresponding objects $\mathcal{A}_{\mathcal{T}}$ was rigorously defined and studied in [BFN1, BFN2, BFN3]. Unfortunately, at this point we don't know how to modify our constructions so that they will depend on $\mathbf{M}$ rather than on $\mathbf{N}$ (but we can check that different ways of representing $\mathbf{M}$ as $T^{*} \mathbf{N}$ (in the cases where it is possible) lead to the same $\mathcal{M}_{C}$ ). We shall sometimes denote the theory $T(\mathbf{M}) / G$ simply by $T(G, \mathbf{N})$.

Here is an interesting source of pairs $(G, \mathbf{N})$ as above. Let $Q$ be an oriented quiver (a.k.a. finite oriented graph) with set of vertices $I$. Let $V$ and $W$ be two finite-dimensional $I$-graded vector spaces over $\mathbb{C}$. Set

$$
G=\prod_{i \in I} \mathrm{GL}\left(V_{i}\right), \quad \mathbf{N}=\left(\bigoplus_{i \rightarrow j} \operatorname{Hom}\left(V_{i}, V_{j}\right)\right) \oplus\left(\bigoplus \operatorname{Hom}\left(V_{i}, W_{i}\right)\right) .
$$

\footnotetext{
${ }^{5}$ By Hamiltonian action we mean a symplectic action with fixed moment map.
} 
Theories associated with such pairs $(G, \mathbf{N})$ are called quiver gauge theories. In the case when $Q$ is a quiver of finite Dynkin type the corresponding Coulomb branches are studied in detail in [BFN2]; we review some of these results in Section 6.

4.6. Toric gauge theories. Let $T \subset\left(\mathbb{C}^{\times}\right)^{n}$ be an algebraic torus. We set $T_{F}=\left(\mathbb{C}^{\times}\right)^{n} / T$ (this is also an algebraic torus). Then $T$ acts naturally on $\mathbb{C}^{n}$, so we can set $\mathbf{N}=\mathbb{C}^{n}, G=T$ in the notation of the previous subsection.

Note that the torus $T_{G}^{\vee}$ also naturally embeds to $\left(\mathbb{C}^{\times}\right)^{n}$ (by dualizing the quotient map $\left.\left(\mathbb{C}^{\times}\right)^{n} \rightarrow T_{F}\right)$. It is then expected that the mirror dual to the theory associated to $\left(T, \mathbb{C}^{n}\right)$ is equal to the theory associated with $\left(T_{F}^{\vee}, \mathbb{C}^{n}\right)$. Note that this implies that the Coulomb branch of the former must be isomorphic to $T^{*} \mathbb{C}^{n} / / T_{F}^{\vee}$ (since this is the Higgs branch of the latter). As was mentioned earlier, in the next Section we are going to give a rigorous definition of Coulomb branches for gauge theories of cotangent type and the above expectation in the toric case is proven in Example 5.5.1.

4.7. Sicilian theories. Let $\Sigma$ be a Riemann surface obtained from a compact Riemann surface $\bar{\Sigma}$ by making $n$ punctures. Let also $G$ be a reductive group. To this data physicists associate a theory $T(\Sigma, G)$ ("Sicilian theory") with an action of $G^{n}$. The construction is by "compactifying" certain 6-dimensional theory (attached to $G$ ) on $\Sigma \times S^{1}$.

One of the key statements from physics is that the theory associated to a sphere with $n$-punctures and the group $G^{\vee}$ is

$$
(\underbrace{(\mathcal{T}[G] \times \cdots \times \mathcal{T}[G])}_{n \text { times }} / G)^{*} .
$$

Here we are gauging the diagonal action of $G$. It has an action of $\left(G^{\vee}\right)^{n}$ for reasons similar to 7 ). There should be in fact a simpler statement (when you start with a theory corresponding to any surface and make an additional puncture), but we are a little confused now about what it is. In particular, it says that functions on

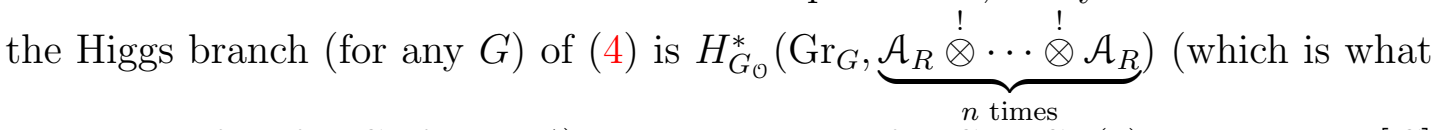
we knew before for $G$ of type $A$ ). More precisely, for $G=G L(r)$ the theory $\mathcal{T}[G]$ is a quiver theory of type $A_{r+1}$ and then the theory $\underbrace{(\mathcal{T}[G] \times \cdots \times \mathcal{T}[G]) / G}_{n \text { times }}$ is the corresponding star-shaped quiver theory. Interested reader can consult [BFN3, Section 6] for more details.

4.8. S-duality vs. derived Satake. Let $\mathcal{T}$ be a theory with $G$-symmetry and let $\mathcal{A}_{\mathcal{T}, G}$ be the corresponding ring object on $\operatorname{Gr}_{G}$. We would like to describe the corresponding data for the S-dual theory $\mathcal{T}^{\vee}$. Let $\Psi_{G}$ denote the derived geometric 
Satake functor going from $D\left(\mathrm{Gr}_{G}\right)$ to the derived category of $G^{\vee}$-equivariant $\mathrm{dg}$ modules over $\operatorname{Sym}\left(\mathfrak{g}^{\vee}[-2]\right)$. Then the cohomology $h^{*}\left(\Psi_{G}\left(\mathcal{A}_{\mathcal{T}, G}\right)\right)$ with grading disregarded can be viewed as a commutative ring object in the cateogory of $G^{\vee}$-equivariant modules over $\operatorname{Sym}\left(\mathfrak{g}^{\vee}\right)$. In other words, $\operatorname{Spec}\left(h^{*}\left(\Psi_{G}\left(\mathcal{A}_{\mathcal{T}, G}\right)\right)\right)$ is a $G^{\vee}$-scheme endowed with a compatible morphism to $\left(\mathfrak{g}^{\vee}\right)^{*}$.

It follows from the results of the previous Section that

$$
\mathcal{M}_{H}\left(\mathcal{T}^{\vee}\right)=\operatorname{Spec}\left(h^{*}\left(\Psi_{G}\left(\mathcal{A}_{\mathcal{T}, G}\right)\right)\right) .
$$

Another (categorical) relationship between the assignment $\mathcal{T} \mapsto \mathcal{T}^{\vee}$ and geometric Langlands duality will be discussed in subsection 7.23.

\section{Coulomb Branches of 3-Dimensional gauge theories}

In this Section we explain how to define Coulomb branches (and some further structures related to flavor symmetry) for gauge theories of cotangent type.

5.1. Summary. Let us summarize what is done in this Section. Let $G$ be a complex connected reductive group and let $\mathbf{N}$ be a representation of it. In this Section we are going to define mathematically the Coulomb branch $\mathcal{M}_{C}(G, \mathbf{N})$ of the gauge theory $\mathcal{T}\left(T^{*} \mathbf{N}\right) / G$. These Coulomb branches will satisfy the following (non-exhaustive) list of properties:

(1) $\mathcal{M}_{C}(G, \mathbf{N})$ is a normal, affine generically symplectic Poisson variety (conjecturally it is singular symplectic but we don't know how to prove this).

(2) Let $T$ be a maximal torus in $G$ and let $W$ be the Weyl group of $G$. Then $\mathcal{M}_{C}(G, \mathbf{N})$ is birationally isomorphic to $\left(T^{*} T^{\vee}\right) / W$. This birational isomorphism is compatible with the Poisson structure. In particular, $\operatorname{dim}\left(\mathcal{M}_{C}(G, \mathbf{N})=\right.$ $2 \operatorname{rank} G$.

(3) There is a natural "integrable system" map $\pi: \mathcal{M}_{C}(G, \mathbf{N}) \rightarrow \mathfrak{t} / W$ which has Lagrangian fibers.

(4) $\mathcal{M}_{C}(G, \mathbf{N})$ is equipped with a canonical quantization; the map $\pi$ also gets quantized.

5.2. General setup. Let $\mathbf{N}$ be a finite dimensional representation of a complex connected reductive group $G$. We consider the moduli space $\mathcal{R}_{G, \mathbf{N}}$ of triples $(\mathcal{P}, \sigma, s)$ where $\mathcal{P}$ is a $G$-bundle on the formal disc $D=\operatorname{Spec} \mathcal{O} ; \sigma$ is a trivialization of $\mathcal{P}$ on the punctured formal disc $D^{*}=\operatorname{Spec} \mathcal{K}$; and $s$ is a section of the associated vector bundle $\mathcal{P}_{\text {triv }}{ }^{G} \mathbf{N}$ on $D^{*}$ such that $s$ extends to a regular section of $\mathcal{P}_{\text {triv }}{ }^{G} \mathbf{N}$ on $D$, and $\sigma(s)$ extends to a regular section of $\mathcal{P} \times \mathbf{N}$ on $D$. In other words, $s$ extends to a regular section of the vector bundle associated to the $G$-bundle glued from $\mathcal{P}$ and $\mathcal{P}_{\text {triv }}$ on the non-separated formal scheme glued from 2 copies of $D$ along $D^{*}$ (raviolo). The group $G_{\mathcal{O}}$ acts on $\mathcal{R}_{G, \mathbf{N}}$ by changing the trivialization $\sigma$, and we have an evident $G_{\mathcal{O}}$-equivariant projection $\mathcal{R}_{G, \mathbf{N}} \rightarrow \operatorname{Gr}_{G}$ forgetting $s$. The fibers of this projection are profinite dimensional vector spaces: the fiber over the base point is $\mathbf{N} \otimes \mathcal{O}$, and all the other fibers are subspaces in $\mathbf{N} \otimes \mathcal{O}$ of finite 
codimension. One may say that $\mathcal{R}_{G, \mathbf{N}}$ is a $G_{\mathcal{O}}$-equvariant "constructible profinite dimensional vector bundle" over $\mathrm{Gr}_{G}$.

5.2.1. Example: affine Steinberg variety. If $\mathbf{N}$ is the adjoint representation $G \curvearrowright$ $\mathfrak{g}$, then $\mathcal{R}_{G, \mathbf{N}}$ is isomorphic to the union $\bigcup_{\lambda \in \Lambda^{+}} T_{\mathrm{Gr}_{G}^{\lambda}}^{*} \mathrm{Gr}_{G}$ of conormal bundles to the $G_{\mathcal{O}^{-}}$orbits in $\mathrm{Gr}_{G}$.

The $G_{\mathcal{O}^{-}}$equivariant Borel-Moore homology $H_{\bullet}^{G_{\mathcal{O}}}\left(\mathcal{R}_{G, \mathbf{N}}\right)$ is defined via the following limiting procedure.

We define $\mathcal{R}_{\leq \lambda}$ as the preimage of $\overline{\mathrm{Gr}}_{G}^{\lambda}$ in $\mathcal{R}:=\mathcal{R}(G, \mathbf{N})$. It suffices to define the $G_{\mathcal{O}^{-}}$-equivariant Borel-Moore homology $H_{\bullet}^{G_{\mathcal{O}}}\left(\mathcal{R}_{\leq \lambda}\right)$ along with the maps $H_{\bullet}^{G_{\mathcal{O}}}\left(\mathcal{R}_{\leq \lambda}\right) \rightarrow H_{\bullet}^{G_{\mathcal{O}}}\left(\mathcal{R}_{\leq \mu}\right)$ for $\lambda \leq \mu$. For a fixed $\lambda$ and $d \gg 0, \mathcal{R}_{\leq \lambda}$ is invariant under the translations by $z^{d} \mathbf{N}_{\mathcal{O}}$, and we denote the quotient by $\mathcal{R}_{<\lambda}^{d}$, so that $\mathcal{R}_{\leq \lambda}=\lim _{\leftarrow} \mathcal{R}_{\leq \lambda}^{d}$. For fixed $\lambda, d$, and $e \gg 0$, the action of $G_{\mathcal{O}}$ on $\mathcal{R}_{\leq \lambda}^{d}$ factors through the action of $G_{\mathcal{O} / z^{e} \mathcal{O}}$. Finally,

$$
H_{\bullet}^{G_{\mathcal{O}}}\left(\mathcal{R}_{\leq \lambda}\right):=H_{G_{\mathcal{O} / z^{e} \mathcal{O}}}^{-\bullet}\left(\mathcal{R}_{\leq \lambda}^{d}, \boldsymbol{\omega}_{\mathcal{R}_{\leq \lambda}^{d}}\right)\left[-2 \operatorname{dim}\left(\mathbf{N}_{\mathcal{O}} / z^{d} \mathbf{N}_{\mathcal{O}}\right)\right]
$$

The cohomological shift means that we are considering the "renormalized" BorelMoore homology, i.e. the cohomology $H_{G_{\mathcal{O}}}^{-\bullet}\left(\mathcal{R}, \boldsymbol{\omega}_{\mathcal{R}}\left[-2 \operatorname{dim} \mathbf{N}_{\mathcal{O}}\right]\right)$.

The $G_{\mathcal{O}}$-equivariant Borel-Moore homology $H_{\bullet}^{G_{\mathcal{O}}}\left(\mathcal{R}_{G, \mathbf{N}}\right)$ forms an associative algebra with respect to the following convolution operation. We consider the diagram

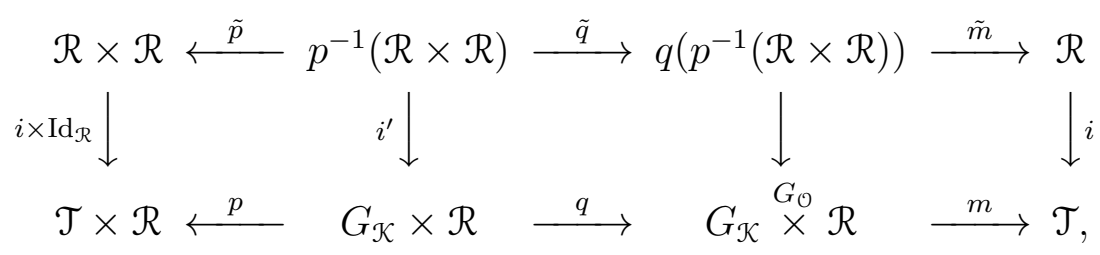

Here $\mathcal{T}:=G_{\mathcal{K}} \stackrel{G_{\mathcal{O}}}{\times} \mathbf{N}_{\mathcal{O}}$, and we have an embedding $\mathcal{T} \hookrightarrow \operatorname{Gr}_{G} \times \mathbf{N}_{\mathcal{K}}$ such that $\mathcal{R}=\mathcal{T} \cap\left(\operatorname{Gr}_{G} \times \mathbf{N}_{\mathcal{O}}\right)$. The embedding $\mathcal{R} \hookrightarrow \mathcal{T}$ is denoted by $i$. The maps in the lower row are given by

$$
\left(g_{1},\left[g_{2}, s\right]\right) \stackrel{q}{\mapsto}\left[g_{1},\left[g_{2}, s\right]\right] \stackrel{m}{\mapsto}\left[g_{1} g_{2}, s\right], \quad\left(g_{1},\left[g_{2}, s\right]\right) \stackrel{p}{\mapsto}\left(\left[g_{1}, g_{2} s\right],\left[g_{2}, s\right]\right),
$$

and all the squares are cartesian (i.e. the upper row consists of closed subvarietes in the lower row, and all the maps in the upper row are induced by the corresponding maps in the lower row). We have the following group actions on the 
terms of the lower row preserving the closed subvarieties in the upper row:

$$
\begin{aligned}
& G_{\mathcal{O}} \times G_{\mathcal{O}} \curvearrowright \mathcal{T} \times \mathcal{R} ;(g, h) \cdot\left(\left[g_{1}, s_{1}\right],\left[g_{2}, s_{2}\right]\right)=\left(\left[g g_{1}, s_{1}\right],\left[h g_{2}, s_{2}\right]\right), \\
& G_{\mathcal{O}} \times G_{\mathcal{O}} \curvearrowright G_{\mathcal{K}} \times \mathcal{R} ;(g, h) \cdot\left(g_{1},\left[g_{2}, s\right]\right)=\left(g g_{1} h^{-1},\left[h g_{2}, s\right]\right), \\
& G_{\mathcal{O}} \curvearrowright G_{\mathcal{K}} \stackrel{G_{\mathcal{O}}}{\times} \mathcal{R} ; g \cdot\left[g_{1},\left[g_{2}, s\right]\right]=\left[g g_{1},\left[g_{2}, s\right]\right], \\
& G_{\mathcal{O}} \curvearrowright \mathcal{T} ; g \cdot\left[g_{1}, s\right]=\left[g g_{1}, s\right] .
\end{aligned}
$$

The morphisms $p, q, m$ (and hence $\tilde{p}, \tilde{q}, \tilde{m}$ ) are equivariant, where we take the first projection $\operatorname{pr}_{1}: G_{\mathcal{O}} \times G_{\mathcal{O}} \rightarrow G_{\mathcal{O}}$ for $q$.

Finally, given two equivariant Borel-Moore homology classes $c_{1}, c_{2} \in H_{\bullet}^{G_{\mathcal{O}}}(\mathcal{R})$, we define their convolution product $c_{1} * c_{2}:=\tilde{m}_{*}\left(\tilde{q}^{*}\right)^{-1} \tilde{p}^{*}\left(c_{1} \otimes c_{2}\right)$.

This algebra is commutative, finitely generated and integral, and its spectrum $\mathcal{M}_{C}(G, \mathbf{N})=\operatorname{Spec} H_{\bullet}^{G_{\mathcal{O}}}\left(\mathcal{R}_{G, \mathbf{N}}\right)$ is an irreducible normal affine variety of dimension $2 \operatorname{rk}(G)$, the Coulomb branch. It is supposed to be a (singular) hyper-Kähler manifold [SW].

Let $T \subset G$ be a Cartan torus with Lie algebra $\mathfrak{t} \subset \mathfrak{g}$. Let $W=N_{G}(T) / T$ be the corresponding Weyl group. Then the equivariant cohomology $H_{G_{\mathcal{O}}}^{\bullet}(\mathrm{pt})=\mathbb{C}[\mathfrak{t} / W]$ forms a subalgebra of $H_{\bullet}^{G_{\mathcal{O}}}\left(\mathcal{R}_{G, \mathbf{N}}\right)$ (a Cartan subalgebra), so we have a projection $\Pi: \mathcal{M}_{C}(G, \mathbf{N}) \rightarrow \mathfrak{t} / W$.

5.2.2. Example. For the adjoint representation $\mathbf{N}=\mathfrak{g}$ considered in 5.2.1, we get $\mathcal{M}_{C}(G, \mathfrak{g})=\left(T^{\vee} \times \mathfrak{t}\right) / W$. For the trivial representation, we get $\mathcal{M}_{C}(G, 0)=$ $\mathfrak{Z}_{\mathfrak{g}^{\vee}}^{G^{\vee}}=\left\{\left(g \in G^{\vee}, \xi \in \Sigma\right): \operatorname{Ad}_{g} \xi=\xi\right\}$, the universal centralizer of the dual group.

Finally, the algebra $H_{\bullet}^{G_{\mathcal{O}}}\left(\mathcal{R}_{G, \mathbf{N}}\right)$ comes equipped with quantization: a $\mathbb{C}[\hbar]$ deformation $\mathbb{C}_{\hbar}\left[\mathcal{M}_{C}(G, \mathbf{N})\right]=H_{\bullet}^{G_{\mathcal{O}} \rtimes \mathbb{C}^{\times}}\left(\mathcal{R}_{G, \mathbf{N}}\right)$ where $\mathbb{C}^{\times}$acts by loop rotations, and $\mathbb{C}[\hbar]=H_{\mathbb{C}^{\times}}^{\bullet}(\mathrm{pt})$. It gives rise to a Poisson bracket on $\mathbb{C}\left[\mathcal{M}_{C}(G, \mathbf{N})\right]$ with an open symplectic leaf, so that $\Pi$ becomes an integrable system: $\mathbb{C}[\mathfrak{t} / W] \subset$ $\mathbb{C}\left[\mathcal{M}_{C}(G, \mathbf{N})\right]$ is a Poisson-commutative polynomial subalgebra with $\operatorname{rk}(G)$ generators.

5.3. Monopole formula. Recall that $\mathcal{R}_{G, \mathbf{N}}$ is a union of (profinite dimensional) vector bundles over $G_{\mathcal{O}^{-}}$orbits in $\mathrm{Gr}_{G}$. The corresponding Cousin spectral sequence converging to $H_{\bullet}^{G_{\mathcal{O}}}\left(\mathcal{R}_{G, \mathbf{N}}\right)$ degenerates and allows to compute the equivariant Poincaré polynomial (or rather Hilbert series)

$$
P_{t}^{G_{\mathcal{O}}}\left(\mathcal{R}_{G, \mathbf{N}}\right)=\sum_{\theta \in \Lambda^{+}} t^{d_{\theta}-2\left\langle\rho^{\vee}, \theta\right\rangle} P_{G}(t ; \theta) .
$$

Here $\operatorname{deg}(t)=2, P_{G}(t ; \theta)=\prod\left(1-t^{d_{i}}\right)^{-1}$ is the Hilbert series of the equivariant cohomology $H_{\operatorname{Stab}_{G}(\theta)}^{\bullet}(\mathrm{pt})\left(d_{i}\right.$ are the degrees of generators of the ring of $\operatorname{Stab}_{G}(\theta)$ invariant functions on its Lie algebra), and $d_{\theta}=\sum_{\chi \in \Lambda_{G}^{\vee}} \max (-\langle\chi, \theta\rangle, 0) \operatorname{dim} \mathbf{N}_{\chi}$. 
This is a slight variation of the monopole formula of [CHZ]. Note that the series (6) may well diverge (even as a formal Laurent series: the space of homology of given degree may be infinite-dimensional), e.g. this is always the case for unframed quiver gauge theories. To ensure its convergence (as a formal Taylor series with the constant term 1) one has to impose the so called 'good' or 'ugly' assumption on the theory. In this case the resulting $\mathbb{N}$-grading on $H_{\bullet}^{G_{\mathcal{O}}}\left(\mathcal{R}_{G, \mathbf{N}}\right)$ gives rise to a $\mathbb{C}^{\times}$-action on $\mathcal{M}_{C}(G, \mathbf{N})$, making it a conical variety with a single (attracting) fixed point.

5.4. Flavor symmetry. Suppose we have an extension $1 \rightarrow G \rightarrow \tilde{G} \rightarrow G_{F} \rightarrow 1$ where $G_{F}$ is a connected reductive group (a flavor group), and the action of $G$ on $\mathbf{N}$ is extended to an action of $\tilde{G}$. Then the action of $G_{\mathcal{O}}$ on $\mathcal{R}_{G, \mathbf{N}}$ extends to an action of $\tilde{G}_{\mathcal{O}}$, and the convolution product defines a commutative algebra structure on the equivariant Borel-Moore homology $H_{\bullet}^{\tilde{G}_{\mathcal{O}}}\left(\mathcal{R}_{G, \mathbf{N}}\right)$. We have the restriction homomorphism $H_{\bullet}^{\tilde{G}_{\mathcal{O}}}\left(\mathcal{R}_{G, \mathbf{N}}\right) \rightarrow H_{\bullet}^{G_{\mathcal{O}}}\left(\mathcal{R}_{G, \mathbf{N}}\right)=H_{\bullet}^{\tilde{G}_{\mathcal{O}}}\left(\mathcal{R}_{G, \mathbf{N}}\right) \otimes_{H_{G_{F}}(\mathrm{pt})} \mathbb{C}$. In other words, $\underline{\mathcal{M}}_{C}(G, \mathbf{N}):=\operatorname{Spec} H_{\bullet}^{\tilde{G}_{\mathcal{O}}}\left(\mathcal{R}_{G, \mathbf{N}}\right)$ is a deformation of $\mathcal{M}_{C}(G, \mathbf{N})$ over Spec $H_{G_{F}}^{\bullet}(\mathrm{pt})=\mathfrak{t}_{F} / W_{F}$.

We will need the following version of this construction. Let $\mathbf{Z} \subset G_{F}$ be a torus embedded into the flavor group. We denote by $\tilde{G}^{\mathrm{Z}}$ the pullback extension $1 \rightarrow G \rightarrow \tilde{G}^{\mathrm{Z}} \rightarrow \mathbf{Z} \rightarrow 1$. We define $\underline{\mathcal{M}}_{C}^{\mathrm{Z}}(G, \mathbf{N}):=\operatorname{Spec} H_{\bullet}^{\tilde{G}_{\mathcal{O}}^{\mathrm{Z}}}\left(\mathcal{R}_{G, \mathbf{N}}\right)$ : a deformation of $\mathcal{M}_{C}(G, \mathbf{N})$ over $\mathfrak{z}:=\operatorname{Spec}_{\mathfrak{Z}}^{\bullet}(\mathrm{pt})$.

Since $\mathcal{M}_{C}(G, \mathbf{N})$ is supposed to be a hyper-Kähler manifold, its flavor deformation should come together with a (partial) resolution. To construct it, we consider the obvious projection $\tilde{\pi}: \mathcal{R}_{\tilde{G}, \mathbf{N}} \rightarrow \mathrm{Gr}_{\tilde{G}} \rightarrow \mathrm{Gr}_{G_{F}}$. Given a dominant coweight $\lambda_{F} \in \Lambda_{F}^{+} \subset \operatorname{Gr}_{G_{F}}$, we set $\mathcal{R}_{\tilde{G}, \mathbf{N}}^{\lambda_{F}}:=\tilde{\pi}^{-1}\left(\lambda_{F}\right)$, and consider the equivariant Borel-Moore homology $H_{\bullet}^{\tilde{G}_{\mathcal{O}}^{\mathrm{Z}}}\left(\mathcal{R}_{\tilde{G}, \mathbf{N}}^{\lambda_{F}}\right)$. It carries a convolution module structure over $H_{\bullet}^{\tilde{G}_{\mathcal{O}}^{\mathrm{Z}}}\left(\mathcal{R}_{G, \mathbf{N}}\right)$. We consider $\underline{\widetilde{\mathcal{M}}}_{C}^{\mathrm{Z}, \lambda_{F}}(G, \mathbf{N}):=\operatorname{Proj}\left(\bigoplus_{n \in \mathbb{N}} H_{\bullet}^{\tilde{G}_{\mathcal{O}}^{\mathrm{Z}}}\left(\mathcal{R}_{\tilde{G}, \mathbf{N}}^{n \lambda_{F}}\right)\right) \stackrel{\varpi}{\longrightarrow}$ $\underline{\mathcal{M}} \underline{\mathrm{Z}}_{C}^{\mathrm{Z}}(G, \mathbf{N})$. We denote $\varpi^{-1}\left(\mathcal{M}_{C}(G, \mathbf{N})\right)$ by $\tilde{\mathcal{M}}_{C}^{\lambda_{F}}(G, \mathbf{N})$. We have $\tilde{\mathcal{M}}_{C}^{\lambda_{F}}(G, \mathbf{N})=$ $\operatorname{Proj}\left(\bigoplus_{n \in \mathbb{N}} H_{\bullet}^{G_{\mathcal{O}}}\left(\mathcal{R}_{\tilde{G}, \mathbf{N}}^{n \lambda_{F}}\right)\right)$.

More generally, for a strictly convex (i.e. not containing nontrivial subgroups) cone $\mathrm{V} \subset \Lambda_{F}^{+}$, we consider the multi projective spectra $\tilde{\mathcal{M}}_{C}^{\mathrm{Z}, \mathrm{V}}(G, \mathbf{N}) \quad:=\operatorname{Proj}\left(\bigoplus_{\lambda_{F} \in \mathrm{V}} H_{\bullet}^{\tilde{G}_{\mathcal{O}}^{\mathrm{Z}}}\left(\mathcal{R}_{\tilde{G}, \mathbf{N}}^{\lambda_{F}}\right)\right) \quad \stackrel{\varpi}{\longrightarrow} \underline{\mathcal{M}}_{C}^{\mathrm{Z}}(G, \mathbf{N}) \quad$ and $\tilde{\mathcal{M}}_{C}^{\vee}(G, \mathbf{N}):=\operatorname{Proj}\left(\bigoplus_{\lambda_{F} \in \mathrm{V}} H_{\bullet}^{G_{\odot}}\left(\mathcal{R}_{\tilde{G}, \mathbf{N}}^{\lambda_{F}}\right)\right) \stackrel{\varpi}{\longrightarrow} \mathcal{M}_{C}(G, \mathbf{N})$.

The following proposition is proved in [BFN1].

Proposition 5.5. Assume that the flavor group is a torus, i.e. we have an exact sequence $1 \rightarrow G \rightarrow \tilde{G} \rightarrow T_{F} \rightarrow 1$. Then the Coulomb branch $\mathcal{M}_{C}(G, \mathbf{N})$ is the Hamiltonian reduction of $\mathcal{M}_{C}(\tilde{G}, \mathbf{N})$ by the action of the dual torus $T_{F}^{\vee}$. 
5.5.1. Example: toric hyper-Kähler manifolds. Consider an exact sequence

$$
0 \rightarrow \mathbb{Z}^{d-n} \stackrel{\alpha}{\rightarrow} \mathbb{Z}^{d \stackrel{\beta}{\rightarrow}} \mathbb{Z}^{n} \rightarrow 0
$$

and the associated sequence

$$
1 \rightarrow G=\left(\mathbb{C}^{\times}\right)^{d-n} \stackrel{\alpha}{\rightarrow} \tilde{G}=\left(\mathbb{C}^{\times}\right)^{d} \stackrel{\beta}{\rightarrow} T_{F}=\left(\mathbb{C}^{\times}\right)^{n} \rightarrow 1
$$

Let $\mathbf{N}=\mathbb{C}^{d}$ considered as a representation of $G$ via $\alpha$. By Proposition 5.5, the Coulomb branch $\mathcal{M}_{C}(G, \mathbf{N})$ is the Hamiltonian reduction of $\mathcal{M}_{C}\left(\left(\mathbb{C}^{\times}\right)^{d}, \mathbb{C}^{d}\right)$ by the action of $T_{F}^{\vee}$. It is easy to see that $\mathcal{M}_{C}\left(\left(\mathbb{C}^{\times}\right)^{d}, \mathbb{C}^{d}\right)=\mathcal{M}_{C}\left(\mathbb{C}^{\times}, \mathbb{C}\right)^{d} \simeq \mathbb{A}^{2 d}$, and hence $\mathcal{M}_{C}(G, \mathbf{N})$ is, by definition, the toric hyper-Kähler manifold associated with the dual sequence of (7) [BiDa].

In particular, if $\mathbf{N}$ is a 1 -dimensional representation of $\mathbb{C}^{\times}$with the character $q^{n}$, then $\mathcal{M}_{C}\left(\mathbb{C}^{\times}, \mathbf{N}\right)$ is the Kleinian surface of type $A_{n-1}$ given by the equation $x y=w^{n}$. If $\mathbf{N}$ is an $n$-dimensional representation of $\mathbb{C}^{\times}$with the character $n q$, then the Coulomb branch $\mathcal{M}_{C}\left(\mathbb{C}^{\times}, \mathbf{N}\right)$ is the same Kleinian surface of type $A_{n-1}$.

5.6. Ring objects in the derived Satake category. Let $\pi$ stand for the projection $\mathcal{R} \rightarrow \operatorname{Gr}_{G}$. Then $\mathcal{A}^{\mathbb{C}^{\times}}:=\pi_{*} \boldsymbol{\omega}_{\mathcal{R}}\left[-2 \operatorname{dim} \mathbf{N}_{\mathcal{O}}\right]$ is an object of $D_{G_{\mathcal{O}} \rtimes \mathbb{C}^{\times}}\left(\operatorname{Gr}_{G}\right)$, and $H_{G_{\mathcal{O}} \rtimes \mathbb{C}^{\times}}^{\bullet}\left(\mathcal{R}, \boldsymbol{\omega}_{\mathcal{R}}\left[-2 \operatorname{dim} \mathbf{N}_{\mathcal{O}}\right]\right)=H_{G_{\mathcal{O}} \rtimes \mathbb{C}^{\times}}\left(\operatorname{Gr}_{G}, \mathcal{A}\right)$. One can equip $\mathcal{A}^{\mathbb{C}^{\times}}$with a structure of a ring object in $D_{G_{\mathcal{O}} \rtimes \mathbb{C}^{\times}}\left(\mathrm{Gr}_{G}\right)$ so that the resulting ring structure on $H_{G_{\mathcal{O}} \rtimes \mathbb{C} \times}^{\bullet}\left(\operatorname{Gr}_{G}, \mathcal{A}^{\mathbb{C}^{\times}}\right)$coincides with the ring structure on $H_{\bullet}^{G_{\mathcal{O}} \rtimes \mathbb{C}^{\times}}(\mathcal{R})$ introduced in Section 5.2. If we forget the loop rotation equivariance, then the resulting ring object $\mathcal{A}$ of $D_{G_{\mathcal{O}}}\left(\mathrm{Gr}_{G}\right)$ is commutative.

Similarly, in the situation of Section 5.4 , we denote $\tilde{\mathcal{R}}:=\mathcal{R}(\tilde{G}, \mathbf{N})$, and consider the composed projection $\tilde{\pi}: \tilde{\mathcal{R}} \rightarrow \mathrm{Gr}_{\tilde{G}} \rightarrow \mathrm{Gr}_{G_{F}}$. We define a ring object $\mathcal{A}_{F}^{\mathbb{C}^{\times}}:=\operatorname{Ind}_{\tilde{G}_{\mathcal{O}} \rtimes \mathbb{C}^{\times}}^{\left(G_{F}\right)_{\mathcal{C}}}\left(\tilde{\pi}_{*} \boldsymbol{\omega}_{\tilde{\mathcal{R}}}\left[-2 \operatorname{dim} \mathbf{N}_{\mathcal{O}}\right] \in D_{\left(G_{F}\right)_{\mathcal{O}} \rtimes \mathbb{C}^{\times}}\left(\operatorname{Gr}_{G_{F}}\right)\right.$, where $\operatorname{Ind}_{\tilde{G}_{\mathcal{O}} \rtimes \mathbb{C}^{\times}}^{\left(G_{F}\right)_{\mathcal{C}}}$ is the functor changing equivariance from $\tilde{G}_{\mathcal{O}} \rtimes \mathbb{C}^{\times}$to $\left(G_{F}\right)_{\mathcal{O}} \rtimes \mathbb{C}^{\times}$. If we forget the loop rotation equivariance, we obtain a commutative ring object $\mathcal{A}_{F} \in D_{\left(G_{F}\right)_{\mathcal{O}}}\left(\mathrm{Gr}_{G_{F}}\right)$. We will also need the fully equivariant ring object $\tilde{\mathcal{A}}_{F}^{\mathbb{C}^{\times}}:=\tilde{\pi}_{*} \boldsymbol{\omega}_{\tilde{\mathcal{R}}}\left[-2 \operatorname{dim} \mathbf{N}_{\mathcal{O}}\right] \in D_{\tilde{G}_{\mathcal{O}} \rtimes \mathbb{C}^{\times}}\left(\mathrm{Gr}_{G_{F}}\right)$.

The ring $\mathbb{C}_{\hbar}\left[\mathcal{M}_{C}(G, \mathbf{N})\right]$ is reconstructed from the ring object $\tilde{\mathcal{A}}_{F}^{\mathbb{C}^{\times}}$ by the following procedure going back to $[\mathrm{ABG}]$. For a flavor coweight $\lambda_{F}$ we denote by $i_{\lambda_{F}}$ the embedding of a $T_{F}$-fixed point $\lambda_{F}$ into $\operatorname{Gr}_{G_{F}}$. Then $\operatorname{Ext}_{D_{\tilde{G}_{\mathcal{O}} \rtimes \mathbb{C}^{\times}}\left(\operatorname{Gr}_{G_{F}}\right)}\left(\mathbf{1}_{\operatorname{Gr}_{G_{F}}}, \tilde{\mathcal{A}}_{F}^{\mathbb{C}^{\times}}\right)=i ! \tilde{\mathcal{A}}_{F}^{\mathbb{C}^{\times}} \simeq H_{\tilde{G}_{\mathcal{O}} \rtimes \mathbb{C}^{\times}}\left(\mathcal{R}, \boldsymbol{\omega}_{\mathcal{R}}\left[-2 \operatorname{dim} \mathbf{N}_{\mathcal{O}}\right]\right)$ by the base change. Given $x, y \in \operatorname{Ext}_{D_{\tilde{G}_{\mathcal{O}} \rtimes \mathbb{C}^{\times}}\left(\operatorname{Gr}_{G_{F}}\right)}\left(\mathbf{1}_{\operatorname{Gr}_{G_{F}}}, \tilde{\mathcal{A}}_{F}^{\mathbb{C}^{\times}}\right)$, we consider $x \star y \in \operatorname{Ext}_{D_{\tilde{G}_{\mathcal{O}} \rtimes \mathbb{C}^{\times}}\left(\operatorname{Gr}_{G_{F}}\right)}\left(\mathbf{1}_{\mathrm{Gr}_{G_{F}}} \star \mathbf{1}_{\mathrm{Gr}_{G_{F}}}, \tilde{\mathcal{A}}_{F}^{\mathbb{C}^{\times}} \star \tilde{\mathcal{A}}_{F}^{\mathbb{C}^{\times}}\right), \quad$ and then apply the isomorphism $\mathbf{1}_{\mathrm{Gr}_{G_{F}}} \simeq \mathbf{1}_{\mathrm{Gr}_{G_{F}}} \star \mathbf{1}_{\mathrm{Gr}_{G_{F}}}$ and the multiplication morphism $\mathrm{m}: \tilde{\mathcal{A}}_{F}^{\mathbb{C}^{\times}} \star \tilde{\mathcal{A}}_{\tilde{\mathscr{A}}^{\times}}^{\mathbb{C}^{\times}} \rightarrow \tilde{\mathcal{A}}_{F}^{\mathbb{C}^{\times}}$in order to obtain $\mathrm{m}(x \star y) \in \operatorname{Ext}_{D_{\tilde{G}_{\mathcal{O}} \rtimes \mathbb{C}^{\times}}\left(\operatorname{Gr}_{G_{F}}\right)}\left(\mathbf{1}_{\mathrm{Gr}_{G_{F}}}, \tilde{\mathcal{A}}_{F}^{\mathbb{C}^{\times}}\right) . \quad$ It is proved in [BFN3] that 
the resulting ring structure on $\operatorname{Ext}_{D_{\tilde{G}_{\mathcal{O}} \rtimes \mathbb{C}^{\times}}\left(\operatorname{Gr}_{G_{F}}\right)}\left(\mathbf{1}_{\operatorname{Gr}_{G_{F}}}, \tilde{\mathcal{A}}_{F}^{\mathbb{C}^{\times}}\right)=H_{\bullet}^{\tilde{G}_{\mathcal{O}} \rtimes \mathbb{C}^{\times}}(\mathcal{R})$ induces the one introduced in Section 5.2 on $H_{\bullet}^{G_{\mathcal{O}} \rtimes \mathbb{C}^{\times}}(\mathcal{R})$. Moreover, a similar construction defines a multiplication $i_{\lambda_{F}}^{!} \overline{\mathcal{A}}_{F}^{\mathbb{C}^{\times}} \otimes i_{\mu_{F}}^{!} \overline{\mathcal{A}}_{F}^{\mathbb{C}^{\times}} \rightarrow i_{\lambda_{F}+\mu_{F}}^{!} \overline{\mathcal{A}}_{F}^{\mathbb{C}^{\times}}$for $\lambda_{F}, \mu_{F} \in \Lambda_{F}^{+}$. Here $\overline{\mathcal{A}}_{F}^{\mathbb{C}^{\times}}=\operatorname{Res}_{\tilde{G}_{\mathcal{O}} \rtimes \mathbb{C}^{\times}}^{G_{\mathcal{O}} \rtimes \mathbb{A}_{F}^{\times}} \tilde{\mathcal{A}}_{F}^{\mathbb{C}^{\times}}$is obtained from $\tilde{\mathcal{A}}_{F}^{\mathbb{C}^{\times}}$applying the functor restricting equivariance from $\tilde{G}_{\mathcal{O}} \rtimes \mathbb{C}^{\times}$to $G_{\mathcal{O}} \rtimes \mathbb{C}^{\times}$. In particular, we get a module structure $i_{0}^{!} \overline{\mathcal{A}}_{F}^{\mathbb{C}^{\times}} \otimes i_{\lambda_{F}}^{!} \overline{\mathcal{A}}_{F}^{\mathbb{C}^{\times}} \rightarrow i_{\lambda_{F}}^{!} \overline{\mathcal{A}}_{F}^{\mathbb{C}^{\times}}$. Note that $i_{0}^{!} \overline{\mathcal{A}}_{F}^{\mathbb{C}^{\times}} \simeq H_{\bullet}^{G_{\mathcal{O}} \rtimes \mathbb{C}^{\times}}(\mathcal{R})$.

5.6.1. Example: The regular sheaf in type $A$. Let $G=\mathrm{GL}\left(\mathbb{C}^{N-1}\right) \times \mathrm{GL}\left(\mathbb{C}^{N-2}\right) \times$ $\ldots \times \mathrm{GL}\left(\mathbb{C}^{1}\right), \quad \tilde{G}=\left(G \times \mathrm{GL}\left(\mathbb{C}^{N}\right)\right) / Z$, where $Z \simeq \mathbb{C}^{\times}$is the diagonal central subgroup. Hence $G_{F}=\operatorname{PGL}\left(\mathbb{C}^{N}\right)$. Furthermore, $\mathbf{N}=\operatorname{Hom}\left(\mathbb{C}^{N}, \mathbb{C}^{N-1}\right) \oplus$ $\operatorname{Hom}\left(\mathbb{C}^{N-1}, \mathbb{C}^{N-2}\right) \oplus \ldots \oplus \operatorname{Hom}\left(\mathbb{C}^{2}, \mathbb{C}^{1}\right)$. It is proved in [BFN3] that $\mathcal{A}_{F}^{\mathbb{C}^{\times}}$is isomorphic to the regular sheaf $\mathcal{A}_{R}^{\mathbb{C}^{\times}} \in D_{\mathrm{PGL}\left(\mathbb{C}^{N}\right)_{\mathcal{O}} \rtimes \mathbb{C}^{\times}}\left(\operatorname{Gr}_{\mathrm{PGL}\left(\mathbb{C}^{N}\right)}\right)$ of Section 3.13.

5.7. Gluing construction. Let $\mathcal{A}_{1}^{\mathbb{C}^{\times}}, \ldots, \mathcal{A}_{n}^{\mathbb{C}^{\times}}$be the ring objects in $D_{G_{\mathcal{O}} \rtimes \mathbb{C}^{\times}}\left(\mathrm{Gr}_{G}\right)$. We denote the ring objects of $D_{G_{\mathcal{O}}}\left(\mathrm{Gr}_{G}\right)$ obtained by forgetting the loop rotation equivariance by $\mathcal{A}_{1}, \ldots, \mathcal{A}_{n}$. Let $i_{\Delta}: \operatorname{Gr}_{G} \hookrightarrow \prod_{k=1}^{n} \operatorname{Gr}_{G}$ be the diagonal embedding. The following proposition is proved in [BFN3].

Proposition 5.8. $\mathcal{A}^{\mathbb{C}^{\times}}:=i_{\Delta}^{!}\left(\bigotimes \mathcal{A}_{k}^{\mathbb{C}^{\times}}\right)$is a ring object in $D_{G_{\mathcal{O}} \rtimes \mathbb{C} \times}\left(\operatorname{Gr}_{G}\right)$. If the ring objects $\mathcal{A}_{1}, \ldots, \mathcal{A}_{n}$ are commutative, then $\mathcal{A}:=i_{\Delta}^{!}\left(\otimes \mathcal{A}_{k}\right) \in D_{G_{\mathcal{O}}}\left(\operatorname{Gr}_{G}\right)$ is a commutative ring object. In particular, the ring $H_{G_{\mathcal{O}}}^{\bullet}\left(\mathrm{Gr}_{G}, \mathcal{A}\right)$ is commutative.

Proof. We have $\nabla \mathrm{m}:\left(\otimes \mathcal{A}_{k}\right) \star\left(\nabla \mathcal{A}_{k}\right)=\bigotimes\left(\mathcal{A}_{k} \star \mathcal{A}_{k}\right) \rightarrow \bigotimes \mathcal{A}_{k}$ from $\mathrm{m}: \mathcal{A}_{k} \star \mathcal{A}_{k} \rightarrow \mathcal{A}_{k}$. Then we apply $i_{\Delta}$. We claim that there is a natural homomorphism

$$
i_{\Delta}^{!}\left(\bigotimes \mathcal{A}_{k}\right) \star i_{\Delta}^{!}\left(\bigotimes \mathcal{A}_{k}\right) \rightarrow i_{\Delta}^{!}\left(\bigotimes\left(\mathcal{A}_{k} \star \mathcal{A}_{k}\right)\right),
$$

hence its composition with $i_{\Delta}(\otimes \mathrm{m})$ gives the desired multiplication homomorphism of $i_{\Delta}^{!}\left(\otimes \mathcal{A}_{k}\right)$. We prove the claim by comparing the convolution diagrams (1) for $\mathrm{Gr}_{G}$ and $\prod_{k} \mathrm{Gr}_{G}$. Since $p, q$ are smooth, $p^{*}, q^{*}$ commute with $i_{\Delta}^{!}$. The last part of the convolution diagram for $G$ and $\prod_{k} G$ is

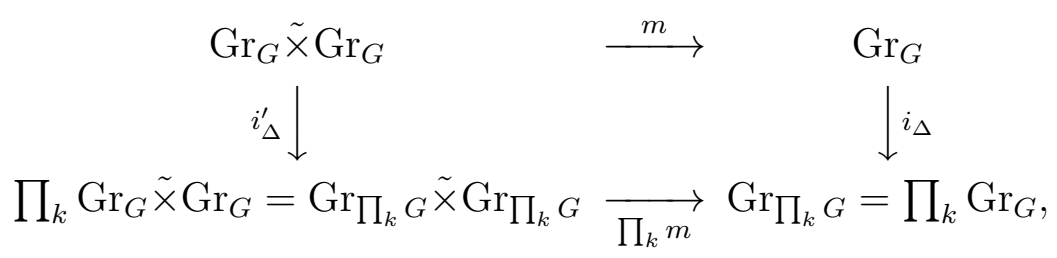

where we denote the diagonal embedding of the left column by $i_{\Delta}^{\prime}$ to distinguish it from the right column. Let $\otimes\left(\mathcal{A}_{k} \tilde{\otimes} \mathcal{A}_{k}\right)$ denote the complex on $\operatorname{Gr}_{\prod_{k} G} \tilde{\times} \operatorname{Gr}_{\prod_{k} G}$ 
obtained in the course of the convolution product for $\prod_{k} G$. We define the homomorphism as

$$
\begin{array}{r}
m_{*} i_{\Delta}^{\prime \prime}\left(\otimes\left(\mathcal{A}_{k} \tilde{\otimes} \mathcal{A}_{k}\right)\right)=m_{*} \stackrel{!}{\bigotimes}\left(\mathcal{A}_{k} \tilde{\otimes} \mathcal{A}_{k}\right) \rightarrow \\
\left.\bigotimes^{!} m_{*}\left(\mathcal{A}_{k} \tilde{\otimes} \mathcal{A}_{k}\right)=i_{\Delta}^{!}\left(\prod_{k} m\right)_{*} \otimes\left(\mathcal{A}_{k} \tilde{\otimes} \mathcal{A}_{k}\right)\right) .
\end{array}
$$

Recall the regular sheaf $\mathcal{A}_{R}^{\mathbb{C}^{\times}}$of Section 3.13. It is equipped with an action of $G^{\vee} \ltimes U_{\hbar}^{[]}$. Hence for any ring object $\mathcal{A}^{\mathbb{C}^{\times}} \in D_{G_{\circlearrowleft} \times \mathbb{C}^{\times}}\left(\operatorname{Gr}_{G}\right)$, the product $\mathcal{A}_{R}^{\mathbb{C}^{\times}} \otimes ! \mathcal{A}^{\mathbb{C}^{\times}}$is also equipped with an action of $G^{\vee} \ltimes U_{\hbar}^{\square]}$. The cohomology ring $H_{G_{\mathcal{O}} \rtimes \mathbb{C}^{\times}}\left(\operatorname{Gr}_{G}, \mathcal{A}_{R}^{\mathbb{C}^{\times}} \otimes ! \mathcal{A}^{\mathbb{C}^{\times}}\right)$is also equipped with an action of $G^{\vee} \ltimes U_{\hbar}^{[]}$. The following proposition is proved in [BFN3] (recall that the autoequivalence $\mathfrak{C}_{G} \vee$ was defined in Section 3.12.1):

Proposition 5.9. For ring objects $\mathcal{A}_{1}^{\mathbb{C}^{\times}}, \mathcal{A}_{2}^{\mathbb{C}^{\times}} \in D_{G_{\mathcal{O}} \rtimes \mathbb{C} \times}\left(\operatorname{Gr}_{G}\right)$, we have

$$
\begin{gathered}
H_{G_{\mathcal{O}} \rtimes \mathbb{C}^{\times}}^{\bullet}\left(\mathrm{Gr}_{G}, \mathcal{A}_{1}^{\mathbb{C}^{\times}} \otimes ! \mathcal{A}_{2}^{\mathbb{C}^{\times}}\right) \simeq \\
H_{G_{\mathcal{O}} \rtimes \mathbb{C}^{\times}}^{\bullet}\left(\mathrm{Gr}_{G}, \mathcal{A}_{R}^{\mathbb{C}^{\times}} \otimes ! \mathcal{A}_{1}^{\mathbb{C}^{\times}}\right) \otimes \mathfrak{C}_{G^{\vee}} H_{G_{\mathcal{O}} \rtimes \mathbb{C}^{\times}}^{\bullet}\left(\mathrm{Gr}_{G}, \mathcal{A}_{R}^{\mathbb{C}^{\times}} \otimes ! \mathcal{A}_{2}^{\mathbb{C}^{\times}}\right) / / \Delta_{G^{\vee}}
\end{gathered}
$$

(quantum Hamiltonian reduction). If the ring objects $\mathcal{A}_{1}, \mathcal{A}_{2} \in D_{G_{\mathcal{O}}}\left(\mathrm{Gr}_{G}\right)$ obtained by forgetting the loop rotation equivariance are commutative, then we have a similar isomorphism of commutative rings:

$$
H_{G_{\mathcal{O}}}^{\bullet}\left(\mathrm{Gr}_{G}, \mathcal{A}_{1} \otimes ! \mathcal{A}_{2}\right) \simeq H_{G_{\mathcal{O}}}^{\bullet}\left(\mathrm{Gr}_{G}, \mathcal{A}_{R} \otimes ! \mathcal{A}_{1}\right) \otimes \mathfrak{C}_{G^{\vee}} H_{G_{\mathcal{O}}}^{\bullet}\left(\mathrm{Gr}_{G}, \mathcal{A}_{R} \otimes ! \mathcal{A}_{2}\right) / / \Delta_{G^{\vee}}
$$

Proof. By rigidity, we have

$$
\begin{gathered}
H_{G_{\mathcal{O}} \rtimes \mathbb{C}^{\times}}^{\bullet}\left(\operatorname{Gr}_{G}, \mathcal{A}_{1}^{\mathbb{C}^{\times}} \otimes ! \mathcal{A}_{2}^{\mathbb{C}^{\times}}\right)=\operatorname{Ext}_{D_{G_{\mathcal{O}} \rtimes \mathbb{C} \times}\left(\operatorname{Gr}_{G}\right)}\left(\mathbb{D} \mathcal{A}_{1}^{\mathbb{C}^{\times}}, \mathcal{A}_{2}^{\mathbb{C}^{\times}}\right) \\
=\operatorname{Ext}_{D_{G_{\mathcal{O}} \rtimes \mathbb{C} \times}\left(\operatorname{Gr}_{G}\right)}\left(\mathbf{1}_{\mathrm{Gr}_{G}}, \mathcal{C}_{G} \mathcal{A}_{1}^{\mathbb{C}^{\times}} \star \mathcal{A}_{2}^{\mathbb{C}^{\times}}\right)= \\
\operatorname{Ext}_{D^{G^{\vee}\left(U_{\hbar}^{[]}\right)}}^{\bullet}\left(U_{\hbar}^{[]}, \mathfrak{C}_{G^{\vee}} \Psi_{\hbar}^{-1}\left(\mathcal{A}_{1}^{\mathbb{C}^{\times}}\right) \otimes_{U_{\hbar}^{[]]}} \Psi_{\hbar}^{-1}\left(\mathcal{A}_{2}^{\mathbb{C}^{\times}}\right)\right) \\
=\operatorname{Ext}_{D^{G^{\vee}}\left(U_{\hbar}^{[]}\right)}^{\bullet}\left(U_{\hbar}^{[]}, \Phi_{\hbar}\left(\mathcal{A}_{1}^{\mathbb{C}^{\times}}\right) \otimes_{U_{\hbar}^{[]}} \mathfrak{C}_{G^{\vee}} \Phi_{\hbar}\left(\mathcal{A}_{2}^{\mathbb{C} \times}\right)\right),
\end{gathered}
$$

(the last equality is Lemma 3.14(b)). Now it is easy to see that $\operatorname{Ext}_{D^{G^{\vee}}\left(U_{\hbar}^{[]}\right)}^{\bullet}\left(U_{\hbar}^{[]}, \Phi_{\hbar}\left(\mathcal{A}_{1}^{\mathbb{C}^{\times}}\right) \otimes_{U_{\hbar}^{[]}} \mathfrak{C}_{G^{\vee}} \Phi_{\hbar}\left(\mathcal{A}_{2}^{\mathbb{C}^{\times}}\right)\right) \quad$ is the hamiltonian reduction $\left(\Phi_{\hbar}\left(\mathcal{A}_{1}^{\mathbb{C}^{\times}}\right) \otimes \mathfrak{C}_{G^{\vee}} \Phi_{\hbar}\left(\mathcal{A}_{2}^{\mathbb{C}^{\times}}\right)\right) / / / \Delta_{G^{\vee}} \quad$ of $\quad \Phi_{\hbar}\left(\mathcal{A}_{1}^{\mathbb{C}^{\times}}\right) \otimes \mathfrak{C}_{G^{\vee}} \Phi_{\hbar}\left(\mathcal{A}_{2}^{\mathbb{C}^{\times}}\right) \quad$ with respect to the diagonal action of $G^{\vee}$. Finally, according to Lemma 3.14, $H_{G_{\mathcal{O}} \rtimes \mathbb{C}^{\times}}^{\bullet}\left(\mathrm{Gr}_{G}, \mathcal{A}_{R}^{\mathbb{C}^{\times}} \otimes ! \mathcal{A}_{1,2}^{\mathbb{C}^{\times}}\right)=\Phi_{\hbar}\left(\mathcal{A}_{1,2}^{\mathbb{C}^{\times}}\right)$. 
5.10. Higgs branches of Sicilian theories. We denote $i_{\Delta}^{!}\left(\mathcal{A}_{R}^{\otimes b}\right)$ by $\mathcal{A}^{b} \in D_{G_{\mathcal{O}}}\left(\mathrm{Gr}_{G}\right)$. It is equipped with an action of $b$ copies of $G^{\vee} \ltimes U_{\hbar}^{[]}$. We denote by $\mathcal{B} \in D_{G_{\mathcal{O}}}\left(\operatorname{Gr}_{G}\right)$ the quantum hamiltonian reduction of $\mathcal{A}^{2}$ by the diagonal action $G^{\vee}$. We expect that $\mathcal{B}$ is isomorphic to $\pi_{*} \boldsymbol{\omega}_{\mathcal{R}_{G, \mathfrak{g}}}\left[-2 \operatorname{dim} \mathfrak{g}_{\mathcal{O}}\right]$ (see Section 5.6 and Example 5.2.1). Finally, we set $\mathcal{B}^{g}:=i_{\Delta}^{!}\left(\mathcal{B}^{\otimes g}\right)$. Then $\mathcal{A}^{b} \otimes^{!} \mathcal{B}^{g}$ is a commutative ring object of $D_{G_{\mathcal{O}}}\left(\mathrm{Gr}_{G}\right)$, and its equivariant cohomology is a commutative ring. We denote by $W_{G}^{g, b}$ its spectrum Spec $H_{G_{\mathcal{O}}}^{\bullet}\left(\mathrm{Gr}_{G}, \mathcal{A}^{b} \otimes^{!} \mathcal{B}^{g}\right)$. It is a Poisson variety equipped with an action of $\left(G^{\vee}\right)^{b}$, the conjectural Higgs branch of a Sicilian theory.

Recall that according to [MT], there is a conjectural functor from the category of 2-bordisms to a category HS of holomorphic symplectic varieties with Hamiltonian group actions. The objects of HS are complex algebraic semisimple groups. A morphism from $G$ to $G^{\prime}$ is a holomorphic symplectic variety $X$ with a $\mathbb{C}^{\times}$-action scaling the symplectic form with weight 2 , together with hamiltonian $G \times G^{\prime}$ action commuting with the $\mathbb{C}^{\times}$-action. For $X \in \operatorname{Mor}\left(G^{\prime}, G\right), Y \in \operatorname{Mor}\left(G, G^{\prime \prime}\right)$, the composition $Y \circ X \in \operatorname{Mor}\left(G^{\prime}, G^{\prime \prime}\right)$ is given by the symplectic reduction of $Y \times X$ by the diagonal $G$-action. The identity morphism in $\operatorname{Mor}(G, G)$ is the cotangent bundle $T^{*} G$ with the left and right action of $G$.

To a complex semisimple group $G$ and a Riemann surface with boundary, physicists associate a $3 d$ Sicilian theory and consider its Higgs branch. It depends only on the topology of the Riemann surface, and gives a functor as above. Such a functor satisfying most of expected properties was constructed recently in [GK]. It follows from Proposition 5.9 that the above $W_{G}^{g, b}$ is associated to the group $G^{\vee}$ and Riemann surface of genus $g$ with $b$ boundary components. It is also proved in $[\mathrm{BFN} 3]$ that $W_{\mathrm{PGL}(2)}^{0,3} \simeq \mathbb{C}^{2} \otimes \mathbb{C}^{2} \otimes \mathbb{C}^{2}$, and $W_{\mathrm{PGL}(3)}^{0,3}$ is the minimal nilpotent orbit of $E_{6}$, while $W_{\mathrm{PGL}(3)}^{1,1}$ is the subregular nilpotent orbit of $G_{2}$, as expected by physicists.

\section{Coulomb BRAnches of $3 d$ Quiver gauge TheOrIES}

6.1. Quiver gauge theories. Let $Q$ be a quiver with $Q_{0}$ the set of vertices, and $Q_{1}$ the set of arrows. An arrow $e \in Q_{1}$ goes from its tail $t(e) \in Q_{0}$ to its head $h(e) \in Q_{0}$. We choose a $Q_{0}$-graded vector spaces $V:=\bigoplus_{j \in Q_{0}} V_{j}$ and $W:=\bigoplus_{j \in Q_{0}} W_{j}$. We set $\mathrm{G}=\mathrm{GL}(V):=\prod_{j \in Q_{0}} \mathrm{GL}\left(V_{j}\right)$. We choose a second grading $W=\bigoplus_{s=1}^{N} W^{(s)}$ compatible with the $Q_{0}$-grading of $W$. We set $\mathrm{G}_{F}$ to be a Levi subgroup $\prod_{s=1}^{N} \prod_{j \in Q_{0}} \mathrm{GL}\left(W_{j}^{(s)}\right)$ of $\mathrm{GL}(W)$, and $\tilde{\mathrm{G}}:=\mathrm{G} \times \mathrm{G}_{F}$.

Remark. $\mathrm{G}$ will be the gauge group in this section. We denote it by $\mathrm{G}$ since we want to use the notation $G$ for some other group.

Finally, we define a central subgroup $\mathrm{Z} \subset \mathrm{G}_{F}$ as follows: $\mathrm{Z}:=\prod_{s=1}^{N} \Delta_{\mathbb{C}^{\times}}^{(s)} \subset$ $\prod_{s=1}^{N} \prod_{j \in Q_{0}} \mathrm{GL}\left(W_{j}^{(s)}\right)$, where $\mathbb{C}^{\times} \cong \Delta_{\mathbb{C}^{\times}}^{(s)} \subset \prod_{j \in Q_{0}} \mathrm{GL}\left(W_{j}^{(s)}\right)$ is the diagonally 
embedded subgroup of scalar matrices. The reductive group $\tilde{G}$ acts naturally on $\mathbf{N}:=\bigoplus_{e \in Q_{1}} \operatorname{Hom}\left(V_{t(e)}, V_{h(e)}\right) \oplus \bigoplus_{j \in Q_{0}} \operatorname{Hom}\left(W_{j}, V_{j}\right)$.

The Higgs branch of the corresponding quiver gauge theory is the Nakajima quiver variety $\mathcal{M}_{H}(\mathrm{G}, \mathbf{N})=\mathfrak{M}(V, W)$. We are interested in the Coulomb branch $\mathcal{M}_{C}(\mathrm{G}, \mathbf{N})$.

6.2. Generalized slices in an affine Grassmannian. Recall the slices $\overline{\mathcal{W}}_{\mu}^{\lambda}$ defined in Section 2.1 for domimant $\mu$. For arbitrary $\mu$ we consider the moduli space $\overline{\mathcal{W}}_{\mu}^{\lambda}$ of the following data:

(a) A $G$-bundle $\mathcal{P}$ on $\mathbb{P}^{1}$.

(b) A trivialization $\sigma:\left.\left.\mathcal{P}_{\text {triv }}\right|_{\mathbb{P}^{1} \backslash\{0\}} \stackrel{\sim}{\longrightarrow} \mathcal{P}\right|_{\mathbb{P}^{1} \backslash\{0\}}$ having a pole of degree $\leq \lambda$ at $0 \in \mathbb{P}^{1}$ (that is defining a point of $\overline{\mathrm{Gr}}_{G}^{\lambda}$ ).

(c) A $B$-structure $\phi$ on $\mathcal{P}$ of degree $w_{0} \mu$ with the fiber $B_{-} \subset G$ at $\infty \in \mathbb{P}^{1}$ (with respect to the trivialization $\sigma$ of $\mathcal{P}$ at $\infty \in \mathbb{P}^{1}$ ). Here $G \supset B_{-} \supset T$ is the Borel subgroup opposite to $B$, and $w_{0} \in W$ is the longest element.

This construction goes back to [FiMi]. The space $\overline{\mathcal{W}}_{\mu}^{\lambda}$ is nonempty iff $\mu \leq \lambda$. In this case it is an irreducible affine normal Cohen-Macaulay variety of dimension $\left\langle 2 \rho^{\vee}, \lambda-\mu\right\rangle$, see [BFN2]. In case $\mu$ is dominant, the two definitions of $\overline{\mathcal{W}}_{\mu}^{\lambda}$ agree. At the other extreme, if $\lambda=0$, then $\overline{\mathcal{W}}_{-\alpha}^{0}$ is nothing but the open zastava space $\stackrel{\circ}{Z}^{-w_{0} \alpha}$. The $T$-fixed point set $\left(\overline{\mathcal{W}}_{\mu}^{\lambda}\right)^{T}$ is nonempty iff the weight space $V_{\mu}^{\lambda}$ is not 0 ; in this case $\left(\overline{\mathcal{W}}_{\mu}^{\lambda}\right)^{T}$ consists of a single point denoted $\mu$.

6.3. Beilinson-Drinfeld slices. Let $\underline{\lambda}=\left(\lambda_{1}, \ldots, \lambda_{N}\right)$ be a collection of dominant coweights of $G$. We consider the moduli space $\overline{\mathcal{W}} \bar{\mu}$ of the following data:

(a) A collection of points $\left(z_{1}, \ldots, z_{N}\right) \in \mathbb{A}^{N}$ on the affine line $\mathbb{A}^{1} \subset \mathbb{P}^{1}$.

(b) A $G$-bundle $\mathcal{P}$ on $\mathbb{P}^{1}$.

(c) A trivialization $\sigma:\left.\left.\mathcal{P}_{\text {triv }}\right|_{\mathbb{P}^{1} \backslash\left\{z_{1}, \ldots, z_{N}\right\}} \stackrel{\sim}{\longrightarrow} \mathcal{P}\right|_{\mathbb{P}^{1} \backslash\left\{z_{1}, \ldots, z_{N}\right\}}$ with a pole of degree $\leq \sum_{s=1}^{N} \lambda_{s} \cdot z_{s}$ on the complement.

(d) A $B$-structure $\phi$ on $\mathcal{P}$ of degree $w_{0} \mu$ with the fiber $B_{-} \subset G$ at $\infty \in \mathbb{P}^{1}$ (with respect to the trivialization $\sigma$ of $\mathcal{P}$ at $\infty \in \mathbb{P}^{1}$ ).

$\overline{\mathcal{W}} \bar{\lambda}$ is nonempty iff $\mu \leq \lambda:=\sum_{s=1}^{N} \lambda_{s}$. In this case it is an irreducible affine normal Cohen-Macaulay variety flat over $\mathbb{A}^{N}$ of relative dimension $\left\langle 2 \rho^{\vee}, \lambda-\mu\right\rangle$, see [BFN2]. The fiber over $N \cdot 0 \in \mathbb{A}^{N}$ is nothing but $\overline{\mathcal{W}}_{\mu}^{\lambda}$.

6.4. Convolution diagram over slices. In the setup of Section 6.3 we consider the moduli space $\underline{\widetilde{\mathcal{W}}} \underline{\underline{\lambda}}$ of the following data:

(a) A collection of points $\left(z_{1}, \ldots, z_{N}\right) \in \mathbb{A}^{N}$ on the affine line $\mathbb{A}^{1} \subset \mathbb{P}^{1}$.

(b) A collection of $G$-bundles $\left(\mathcal{P}_{1}, \ldots, \mathcal{P}_{N}\right)$ on $\mathbb{P}^{1}$.

(c) A collection of isomorphisms $\sigma_{s}:\left.\left.\mathcal{P}_{s-1}\right|_{\mathbb{P}^{1} \backslash\left\{z_{s}\right\}} \stackrel{\sim}{\longrightarrow} \mathcal{P}_{s}\right|_{\mathbb{P}^{1} \backslash\left\{z_{s}\right\}}$ with a pole of degree $\leq \lambda_{s}$ at $z_{s}$. Here $1 \leq s \leq N$, and $\mathcal{P}_{0}:=\mathcal{P}_{\text {triv }}$. 
(d) A $B$-structure $\phi$ on $\mathcal{P}_{N}$ of degree $w_{0} \mu$ with the fiber $B_{-} \subset G$ at $\infty \in \mathbb{P}^{1}$ (with respect to the trivialization $\sigma_{N} \circ \ldots \circ \sigma_{1}$ of $\mathcal{P}_{N}$ at $\infty \in \mathbb{P}^{1}$ ).

A natural projection $\varpi: \widetilde{\mathcal{W}}_{\mu}^{\lambda} \rightarrow \underline{\mathcal{W}_{\mu}} \frac{\lambda}{\mu}$ sends $\left(\mathcal{P}_{1}, \ldots, \mathcal{P}_{N}, \sigma_{1}, \ldots, \sigma_{N}\right)$ to $\left(\mathcal{P}_{N}, \sigma_{N} \circ\right.$ $\left.\ldots \circ \sigma_{1}\right)$. We denote $\varpi^{-1}\left(\overline{\mathcal{W}}_{\mu}^{\lambda}\right)$ by $\widetilde{\mathcal{W}} \frac{\lambda}{\mu}$. Then we expect that $\varpi: \widetilde{\mathcal{W}} \frac{\lambda}{\mu} \rightarrow \overline{\mathcal{W}}_{\mu}^{\lambda}$ is stratified semismall.

6.5. Slices as Coulomb branches. Let now $G$ be an adjoint simple simply laced algebraic group. We choose an orientation $\Omega$ of its Dynkin graph (of type $A D E)$, and denote by $I$ its set of vertices. Given an $I$-graded vector space $W$ we encode its dimension by a dominant coweight $\lambda:=\sum_{i \in I} \operatorname{dim}\left(W_{i}\right) \omega_{i} \in \Lambda^{+}$ of $G$. Given an $I$-graded vector space $V$ we encode its dimension by a positive coroot combination $\alpha:=\sum_{i \in I} \operatorname{dim}\left(V_{i}\right) \alpha_{i} \in \Lambda_{+}$. We set $\mu:=\lambda-\alpha \in \Lambda$. Given a direct sum decomposition $W=\bigoplus_{s=1}^{N} W^{(s)}$ compatible with the $I$-grading of $W$ as in Section 6.1 , we set $\lambda_{s}:=\sum_{i \in I} \operatorname{dim}\left(W_{i}^{(s)}\right) \omega_{i} \in \Lambda^{+}$, and finally, $\underline{\lambda}:=$ $\left(\lambda_{1}, \ldots, \lambda_{N}\right)$.

Recall the notations of Section 5.4. Since the flavor group $\mathrm{G}_{F}$ is a Levi subgroup of $\mathrm{GL}(W)$, its weight lattice is naturally identified with $\mathbb{Z}^{\operatorname{dim} W}$. More precisely, we choose a basis $w_{1}, \ldots, w_{\operatorname{dim} W}$ of $W$ such that any $W_{i}, i \in I$, and $W^{(s)}, 1 \leq s \leq N$, is spanned by a subset of the basis, and we assume the following monotonicity condition: if for $1 \leq a<b<c \leq \operatorname{dim} W$ we have $w_{a}, w_{b} \in W^{(s)}$ for certain $s$, then $w_{b} \in W^{(s)}$ as well. We define a strictly convex cone $\mathrm{V}=\left\{\left(n_{1}, \ldots, n_{\operatorname{dim} W}\right)\right\} \subset$ $\Lambda_{F}^{+} \subset \mathbb{Z}^{\operatorname{dim} W}$ by the following conditions: (a) if $w_{k} \in W^{(s)}, w_{l} \in W^{(t)}$, and $s<t$, then $n_{k} \geq n_{l} \geq 0$; (b) if $w_{k}, w_{l} \in W^{(s)}$, then $n_{k}=n_{l}$. The following theorem is proved in [BFN2, BFN4] by the fixed point localization and reduction to calculations in rank 1 :

Theorem 6.6. We have isomorphisms

$\overline{\mathcal{W}}_{\mu}^{\lambda} \stackrel{\sim}{\longrightarrow} \mathcal{M}_{C}(\mathrm{G}, \mathbf{N}), \underline{\overline{\mathcal{W}}_{\mu}^{\lambda}} \stackrel{\sim}{\longrightarrow} \underline{\mathcal{M}}_{C}^{\mathrm{Z}}(\mathrm{G}, \mathbf{N}), \underline{\widetilde{\mathcal{W}}_{\mu}^{\lambda}} \stackrel{\sim}{\longrightarrow} \underline{\mathcal{M}}_{C}^{\mathrm{Z}, \mathrm{V}}(\mathrm{G}, \mathbf{N}), \quad \widetilde{\mathcal{W}}_{\mu}^{\lambda} \stackrel{\sim}{\longrightarrow} \widetilde{\mathcal{M}}_{C}^{\mathrm{V}}(\mathrm{G}, \mathbf{N})$.

6.7. Further examples. Let now $Q$ be an affine quiver of type $\tilde{A} \tilde{D} \tilde{E}$; the framing $W$ is 1-dimensional concentrated at the extending vertex; and the dimension of $V$ is $d$ times the minimal imaginary coroot $\delta$. Then it is expected that $\mathcal{M}_{C}(\mathrm{G}, \mathbf{N})$ is isomorphic to the Uhlenbeck (partial) compactification $\mathcal{U}_{G}^{d}\left(\mathbb{A}^{2}\right)[\mathrm{BFG}]$ of the moduli space of $G$-bundles on $\mathbb{P}^{2}$ trivialized at $\mathbb{P}_{\infty}^{1}$, of second Chern class $d$. This is proved for $G=\mathrm{SL}(N)$ in $[\mathrm{NT}]$.

Furthermore, let $Q$ be a star-shaped quiver with $b$ legs of length $N$ each, and with $g$ loop-edges at the central vertex. The framing is trivial, and the dimension of $V$ along each leg, starting at the outer end, is $1,2, \ldots, N-1, N$ (with $N$ at the central vertex). Contrary to the general setup in Section 6.1, we define $G$ as the quotient of $\mathrm{GL}(V)$ by the diagonal central subgroup $\mathbb{C}^{\times}$(acting trivially on $\mathbf{N}$ ). Then according to [BFN3], $\mathcal{M}_{C}(\mathrm{G}, \mathbf{N})$ is isomorphic to $W_{\mathrm{PGL}(N)}^{g, b}$ of Section 5.10. 


\section{More Physics: topological twists of 3D N=4 QFT AND CATEGORICAL CONSTRUCTIONS}

The constructions of this Section are mostly conjectural. The main idea of this Section is given by equation (10) which is due to T. Dimofte, D. Gaiotto, J. Hilburn and P. Yoo. We discuss some interesting corollaries of this equation.

7.1. Extended topological field theories. Physical quantum field theories usually depend on a choice of metric on the space-time. The theory is called topological if all the quantities (e.g. corelation functions) are independent of the metric (however, look at the warning at the end of the next subsection). Mathematically, the axioms of a topological QFT were first formulated by Atiyah (cf. [At]). Roughly speaking, a topological quantum field theory in dimension $d$ consists of the following data:

(a) A complex number $Z\left(M^{d}\right)$ for every closed $d$-dimensional manifold $M^{d}$;

(b) A space $Z\left(M^{d-1}\right)$ for every closed $(d-1)$-dimensional manifold $M^{d-1}$;

(c) A vector in $Z(\partial M)$ for every compact oriented $d$-dimensional manifold $M$ with boundary $\partial M$.

These data must satisfy certain list of standard axioms; we refer the reader to [At] for details. In addition, one can consider a richer structure called extended topological field theory. This structure in addition to (a), (b) and (c) as above must associate $k$-category $Z\left(M^{d-k-1}\right)$ to a closed manifold $M^{d-k-1}$ of dimension $d-k-1$. It should also associate an object of the $k$-category $Z(\partial M)$ to every compact oriented manifold $M$ of dimension $d-k$; more generally, there is a structure associated with every manifold with corners of dimension $\leq d$. We refer the reader to [Lur1] for details about extended topological field theories. In the sequel we shall be mostly concerned with the case $d=3$. In this case one is supposed to associate a (usual) category to the circle $S^{1}$. Physicists call it the category of line operators.

7.2. Topological twists of $\mathbf{3 d} \mathbf{N}=\mathbf{4}$ theories. Physical quantum field theories are usually not topological. However, sometimes physicists can produce a universal procedure which associates a topological field theory to a physical theory with enough super-symmetry. Since in these notes we are not discussing what a quantum field theory really is, we can't discuss what a topological twist really is. Physicists say that any $3 \mathrm{~d} N=4$ theory with some mild additional structure ${ }^{6}$ must have two topological twists (we'll call them Coulomb and Higgs twists, although physicists often call them $A$ and $B$ twists by analogy with similar construction for 2-dimensional field theories). These twists must be interchanged by the $3 \mathrm{~d}$ mirror symmetry operation mentioned in Section 4.

\footnotetext{
${ }^{6}$ The nature of this additional structure will become more clear in Subsection 7.7.
} 
7.3. Warning. The twists are topological only in some weak sense. Namely, in principle as was mentioned above in a topological field theory everything (e.g. correlators) should be independent of the metric (i.e. only depend on the topology of the relevant space-time). In a weakly topological field theory everything should be metric-independent only locally. This issue will be ignored in this section since we are only going to discuss some pretty robust things but it is actually important if one wants to understand some finer aspects.

7.4. The category of line operators in a topologically twisted $3 \mathrm{~d} N=\mathbf{4}$ theory. To a 3d TFT one should be able to attach a "category of line operators" (i.e. this is the category one attaches to a circle in terms of the previous subsection). Morever, since the circle $S^{1}$ is the boundary of a canonical 2-dimensional manifold: the 2-dimensional disc, this category should come equipped with a canonical object. In this Section we would like to suggest a construction of these categories together with the above object for a wide class of topologically twisted $3 \mathrm{~d} N=4$ theories (we learned the idea of this construction from T. Dimofte, D. Gaiotto, J. Hilburn and P. Yoo who can actually derive this construction from physical considerations. To the best of our knowledge their paper on the subject is forthcoming).

A priori the above categories of line operators should be $\mathbb{Z}_{2}$-graded. However, as was mentioned above, in order to define the relevant topological twists one needs to choose some mild additional structure on the theory (we explain this additional structure in series of examples in Subsection 7.7). So we are actually going to think about them as $\mathbb{Z}$-graded categories (in fact, as dg-categories). But we should keep in mind that if we choose this additional structure in a different way, then a priori we should get different $\mathbb{Z}$-graded categories but with the same underlying $\mathbb{Z}_{2}$-graded categories.

Since a $3 \mathrm{~d} N=4$ theory is supposed to have two topological twists which we call Coulomb and Higgs, we shall denote the corresponding categories of line operators by $\mathcal{C}_{C}, \mathcal{C}_{H}$. As was mentioned above, filling the circle with a disc should produce canonical objects $\mathcal{F}_{C}, \mathcal{F}_{H}$.

Remark for an advanced reader. In principle in a true TQFT the category of line operators should be an $E_{2}$-category (cf. [Lur2]). There is a closely related notion of factorizable category (in the $D$-module sense), a.k.a. chiral category, cf. [Ras]. In fact, the categories we are going to construct will be factorizable categories (and the canonical object, corresponding to the $2 \mathrm{~d}$ disc will be a factorizable object). The fact that we get factorizable categories as opposed to $E_{2}$-categories is related to the warning in subsection 7.8 .

The relation between these structures and what we have discussed in the previous Sections is that one should have

$$
\operatorname{Ext}^{*}\left(\mathcal{F}_{C}, \mathcal{F}_{C}\right)=\mathbb{C}\left[\mathcal{M}_{C}\right]
$$


and

$$
\operatorname{Ext}^{*}\left(\mathcal{F}_{H}, \mathcal{F}_{H}\right)=\mathbb{C}\left[\mathcal{M}_{H}\right]
$$

Remark. It can be shown that for any factorization category $\mathcal{C}$ and a factorization object $\mathcal{F}$ the algebra $\operatorname{Ext}^{*}(\mathcal{F}, \mathcal{F})$ is graded commutative.

When we need to emphasize dependence on a theory $\mathcal{T}$, we shall write $\mathcal{C}_{C}(\mathcal{T}), \mathcal{F}_{C}(\mathcal{T})$ etc. The mirror symmetry conjecture then says

Conjecture 7.5. The category $\mathcal{C}_{C}(\mathcal{T})$ is equivalent to $\mathcal{C}_{H}\left(\mathcal{T}^{*}\right)$ (and the same with $C$ and $H$ interchanged). Under this equivalence the object $\mathcal{F}_{C}(\mathcal{T})$ goes over to $\mathcal{F}_{H}\left(\mathcal{T}^{*}\right)$.

7.6. Generalities on $D$-modules and de Rham pre-stacks. In what follows we'll need to work with various categories of sheaves on spaces which are little more general than usual schemes or stacks. Namely, we need to discuss de Rham pre-stacks and various categories of sheaves related to them. Our main reference for the subject is [GaiRo].

Let $S$ be a smooth scheme of finite type over $\mathbb{C}$. Then one can define certain pre-stack (i.e. a functor from $\mathbb{C}$-algebras to sets) $S_{d R}$ which is called the de Rham pre-stack of $S$. Informally it is defined as the quotient of $S$ by infinitesimal automorphisms. Moreover, this definition can be extended to all schemes, stacks or even dg-stacks of finite type over $\mathbb{C}$. A key property of $S_{d R}$ is that the category of quasi-coherent sheaves on $S_{d R}$ is the same as the category of $D$-modules on $S{ }^{7}$ In addition for a target stack $y$ one can consider the mapping space $\operatorname{Maps}\left(S_{d R}, y\right)$. Here are two important examples:

(1) Let $y=\mathbb{A}^{1}$. Then $\operatorname{Maps}\left(S_{d R}, y\right)$ is the de Rham cohomology of $S$ considered as a dg-scheme.

(2) Let $y=$ pt $/ G$ where $G$ is an algebraic group. Then $\operatorname{Maps}\left(S_{d R}, y\right)$ is the stack of $G$-local systems on $S$ (i.e. the stack classifying $G$-bundles on $S$ endowed with a flat connection).

In the sequel we'll need to apply these constructions to $S$ being either the formal $\operatorname{disc} \mathcal{D}=\operatorname{Spec}(\mathcal{O})$ or the punctured disc $\mathcal{D}^{*}=\operatorname{Spec}(\mathcal{K})$. This is not formally a special case of the above as some completion issues arise if one tries to spell out a careful definition. However, with some extra care all definitions can be extended to this case. This is done in [Gai3].

7.7. Construction of the categories in the cotangent case. It is expected that one can attach the above theories and categories to any symplectic dg-stack $X$. It is now easy to spell out the additional structure on the theory that one needs in order to define the two topological twists in terms of the stack $\mathcal{X}$. Namely, one

\footnotetext{
${ }^{7}$ Because we plunge ourselves into world of derived algebraic geometry here, it doesn't make sense to talk about either quasi-coherent sheaves or $D$-modules as an abelian category: only the corresponding derived category makes sense.
} 
needs a $\mathbb{C}^{\times}$-action on $\mathcal{X}$ with respect to which the symplectic form $\omega$ has weight 2.

We shall actually assume that $\mathcal{X}=T^{*} y$ where $y$ is a smooth stack; in this case the above $\mathbb{C}^{\times}$-action is automatic (we can just use the square of the standard $\mathbb{C}^{\times}$-action on the cotangent fibers). We shall denote this theory by $\mathcal{T}(y)$.

The following construction is due to T. Dimofte, D. Gaiotto, J. Hilburn and P. Yoo (private communication). Namely, let us set

$$
\mathcal{C}_{C}=D-\bmod \left(\operatorname{Maps}\left(\mathcal{D}^{*}, y\right)\right) ; \quad \mathcal{C}_{H}=\operatorname{QCoh}\left(\operatorname{Maps}\left(\mathcal{D}_{d R}^{*}, y\right)\right) .
$$

Let us stress that both $D-\bmod$ and QCoh mean the corresponding derived categories.

Let now $\pi_{C}: \operatorname{Maps}\left(\mathcal{D}_{d R}, y\right) \rightarrow \operatorname{Maps}\left(\mathcal{D}_{d R}^{*}, y\right)$ be the natural map; similarly we define $\pi_{H}$. Then, we set

$$
\mathcal{F}_{H}=\left(\pi_{H}\right)_{*} \mathcal{O}_{\operatorname{Maps}\left(\mathcal{D}_{d R}, y\right)} ; \quad \mathcal{F}_{C}=\left(\pi_{C}\right) ! \mathcal{O}_{\operatorname{Maps}(\mathcal{D}, y)} .
$$

7.8. A very important warning. The above suggestion is probably only an approximation of a true statement. In fact, we believe that the suggestion is fine for $\mathcal{C}_{C}$; however, for $\mathcal{C}_{H}$ some modifications might be necessary. Let, for example (for simplicity), $z$ be a dg-stack of finite type over $\mathbb{C}$. Then following [ArGa] in addition to the category $\mathrm{QCoh}(\mathcal{Z})$ one can also study the derived category $\operatorname{Ind} \operatorname{Coh}(z)$ of ind-coherent sheaves on $z$. The two categories coincide when $z$ is a smooth classical (i.e. not dg) stack. But for more general $z$ these categories are different. This can be seen as follows: the compact objects of $\operatorname{Ind} \operatorname{Coh}(z)$ are by definition finite complexes with coherent cohomology, while the compact objects of $\mathrm{QCoh}(z)$ are finite perfect complexes. Moreover, assume that $z$ is locally a complete intersection. Then in [ArGa] the authors define certain stack $\operatorname{Sing}(z)$ endowed with a representable map $\operatorname{Sing}(z) \rightarrow z$, which is an isomorphism when $z$ is a smooth classical stack. Moreover, the fibers of this map are vector spaces; in particular, there is a natural $\mathbb{C}^{\times}$-action on the fibers whose stack of fixed points is naturally identified with $\mathcal{Z}$. Given a closed conical substack $\mathcal{W} \subset$

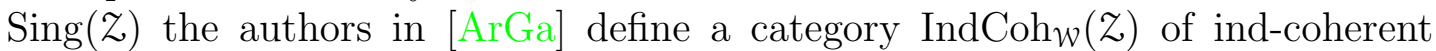
sheaves with singular support in $\mathcal{W}$. These categories in some sense interpolate between $\mathrm{QCoh}(\mathcal{Z})$ and $\operatorname{Ind} \operatorname{Coh}(\mathcal{Z})$ : namely, when $\mathcal{W}=\mathcal{Z}$ (the zero section of the morphism $\operatorname{Sing}(Z) \rightarrow Z$ ) we have $\operatorname{Ind}_{\mathcal{Z}} \operatorname{Coh}_{\mathcal{W}}(z)=\mathrm{QCoh}(\boldsymbol{Z})$, and when $\mathcal{W}=$ $\operatorname{Sing}(z)$ we have $\operatorname{Ind} \operatorname{Coh}_{\mathcal{W}}(z)=\operatorname{Ind} \operatorname{Coh}(z) .^{8}$

We think that suggestion (10) is only "the first approximation" to the right statement. More precisely, we believe that it is literally the right suggestion for the category $\mathcal{C}_{C}$, but for the category $\mathcal{C}_{H}$ one has to be more careful. We believe that the correct definition of the category $\mathcal{C}_{H}$ in the above context should actually be

\footnotetext{
${ }^{8}$ The reader should be warned that although we have a natural functor $\operatorname{IndCoh}_{\mathcal{W}}(Z) \rightarrow$ $\operatorname{IndCoh}(Z)$, this functor is not fully faithful, so $\operatorname{IndCoh}_{\mathcal{W}}(Z)$ is not a full subcategory of $\operatorname{Ind} \operatorname{Coh}(z)$.
} 
$\left.\operatorname{IndCoh} \operatorname{Waps}_{\mathcal{W}}\left(\mathcal{D}_{d R}^{*}, \boldsymbol{y}\right)\right)$ for a particular choice of $\mathcal{W}$ (very often $\mathcal{W}$ will actually be the zero section but probably not always); at this moment we don't know how to specify $\mathcal{W}$ in the above generality. The purpose of the rest of the Section will be to explain some general picture, so in what follows we are going to ignore this subtlety, i.e. we shall proceed with the suggestion $\mathcal{C}_{H}=\operatorname{QCoh}\left(\operatorname{Maps}\left(\mathcal{D}_{d R}^{*}, y\right)\right)$ as stated. But the reader should keep in mind that in certain cases it should be replaced by $\operatorname{IndCoh} \operatorname{Cop}_{\mathcal{W}}\left(\operatorname{Maps}\left(\mathcal{D}_{d R}^{*}, y\right)\right.$ ) (this issue will become important when we formulate some rigorous conjectures (cf. for example the discussion before Conjecture 7.21).

7.9. Remarks about rigorous definitions. Since the above mapping spaces are often genuinely infinite-dimensional, we need to discuss why the above categories make sense. First, the category of $D$-modules on arbitrary pre-stack is discussed in [Ras-dm]. In fact, in loc. cit. the author defines two versions of this category, which are denoted by $D^{!}$and $D^{*}$ (these two categories are dual to each other). For the purposes of these notes we need to work with $D^{*}$ : for example since it is this category for which the functor of direct image is well-defined.

The category $\mathrm{QCoh}(z)$ is well-defined for any pre-stack $z$; however in such generality it might be difficult to work with. However, we would like to note that $\operatorname{Maps}\left(\mathcal{D}_{d R}^{*}, y\right)$ is typically a very manageable object. For example, when $y$ is a scheme of finite type over $\mathbb{C}$ it follows from Conjecture 7.12 below that $\operatorname{Maps}\left(\mathcal{D}_{d R}^{*}, y\right)$ is a dg-scheme of finite type over $\mathbb{C}$, so QCoh is "classical" (modulo the fact that we have to work with commutative dg-algebras as opposed to usual commutative algebras). When $y$ is a stack, the definition is a bit less explicit; however, we claim that the definition is easy when $y$ is of the form $\mathcal{S} / G$ where $\mathcal{S}$ is an affine scheme and $G$ is a reductive group. For example, when $y=p t / G$ we have $\operatorname{Maps}\left(\mathcal{D}_{d R}^{*}, y\right)=\operatorname{LocSyS}_{G}\left(\mathcal{D}^{*}\right)$ : the stack of $G$-local systems (i.e. principal $G$-bundles with a connection on $\left.\mathcal{D}^{*}\right)$, and $\mathrm{QCoh}\left(\operatorname{LocSyS}_{G}\left(\mathcal{D}^{*}\right)\right)$ is a well-studied object in (local) geometric Langlands correspondence.

Here is another reason why we want $y$ to be of the above form. The map $\pi_{H}$ is actually always a closed embedding, so we could write $\left(\pi_{H}\right)$ ! instead of $\left(\pi_{H}\right)_{*}$. On the other hand, the functor $\left(\pi_{C}\right)$ ! is a priori not well defined, at least it is not defined for an arbitrary morphism. However, it is well-defined if the morphism $\pi_{C}$ is ind-proper. In what follows we shall always assume that the stack $y$ is such that it is the case. This condition is not always satisfied but it is also not super-restrictive as follows from the next exercise.

Exercise. (a) Show that $\pi_{C}$ is a closed embedding if $y$ is a scheme.

(b) Show that if $y=\mathcal{S} / G$ where $\mathcal{S}$ is an affine scheme and $G$ is a reductive algebraic group then the morphism $\pi_{C}$ is ind-proper.

(c) Show that (b) might become false if we drop either the assumption that $\mathcal{S}$ is affine or the assumption that $G$ is reductive. 
We shall denote the corresponding categories (10) and objects (11) simply by $\mathcal{C}_{C}(y), \mathcal{F}_{C}(y)$ etc. Note that these categories are $\mathbb{Z}$-graded. The above arguments then suggest the following

Conjecture 7.10. Let $y, y^{\prime}$ be two stacks such that $T^{*} y$ is isomorphic to $T^{*} y^{\prime}$ as a symplectic dg-stack. Then the corresponding $\mathbb{Z}_{2}$-graded versions of $\mathcal{C}_{C}(y)$ and $\mathcal{C}_{C}\left(y^{\prime}\right)$ are equivalent as $\mathbb{Z}_{2}$-graded factorization categories; this equivalence sends $\mathcal{F}_{C}(y)$ to $\mathcal{F}_{C}\left(y^{\prime}\right)$. Similar statement holds for $\mathcal{C}_{H}$.

7.11. Small loops. In fact, one can demistify the category $\mathcal{C}_{H}(y)$ a little bit which makes it quite computable. First of all, with the correct definition it is easy to see that $\operatorname{Maps}\left(\mathcal{D}_{d R}, y\right)$ is equivalent to $y$ (for any $y$ ). ${ }^{9}$ Now, given $y$ let us define another dg-stack $L y$ (we shall call it "small loops" into $y$ ) by setting

$$
L y=y \underset{y \times y}{\times} y
$$

where in the above equation both maps $y \rightarrow y \times y$ are equal to the diagonal map. ${ }^{10}$ We have a natural map $y \rightarrow L y$.

Conjecture 7.12. (1) Let $y$ be a scheme. Then $L y$ and $\operatorname{Maps}\left(\mathcal{D}_{d R}^{*}, y\right)$ are isomorphic (and this isomorphism is compatible with the map from $y=$ $\operatorname{Maps}\left(\mathcal{D}_{d R}, y\right)$ into both).

(2) Let $y$ be a stack. Then the formal neighbourhoods of $y=\operatorname{Maps}\left(\mathcal{D}_{d R}, y\right)$ in $\operatorname{Maps}\left(\mathcal{D}_{d R}^{*}, y\right)$ and in $L y$ are equivalent.

The proof of Conjecture 7.12 will be written in a different publication. In what follows we shall assume Conjecture 7.12.

7.13. Remark. If $y$ is a scheme then it is easy to see that both $\operatorname{Maps}\left(\mathcal{D}_{d R}^{*}, y\right)$ and $L y$ are dg-extensions of $y$ (i.e. they are dg-schemes whose underlying classical scheme is $y$ ), so if the statement of Conjecture 7.12 holds on the level of formal neighbourhoods then in fact we have $L y=\operatorname{Maps}\left(\mathcal{D}_{d R}^{*}, y\right)$. This is not the case for stacks. Namely, let $G$ be a reductive algebraic group and let $y=p t / G$. Then it is easy to see that $\operatorname{Maps}\left(\mathcal{D}_{d R}^{*}, y\right)$ is the stack $\operatorname{LocSys}_{G}\left(\mathcal{D}^{*}\right)$ of $G$-local systems on $\mathcal{D}^{*}$ (i.e. principal $G$-bundles on $\mathcal{D}^{*}$ with a connection).

Exercise. Show that for $y=$ pt $/ G$ we have $L y=G / \operatorname{Ad}(G)$ (i.e. quotient of $G$ by itself with respect to the adjoint action). Show that $G / \operatorname{Ad}(G)$ is not equivalent to $\operatorname{LocSys}_{G}\left(\mathcal{D}^{*}\right)$ but the formal neighbourhoods of pt/ $G$ in both are equivalent (the embedding of pt/ $G$ into $\operatorname{LocSys}_{G}\left(\mathcal{D}^{*}\right)$ corresponds to the trivial local system).

\footnotetext{
${ }^{9}$ Here we see that $\operatorname{Maps}\left(\mathcal{D}_{d R}, y\right)$ should be defined with some extra care. Namely, if we just used the naive definition then the equivalence $\operatorname{Maps}\left(\mathcal{D}_{d R}, y\right) \simeq y$ would imply that $\mathcal{D}_{d R}=\mathrm{pt}$ which is far from being the case.

${ }^{10}$ Here we want to stress once again that all fibered products must be understood in the dg-sense!
} 
7.14. An example. The significance of Conjecture 7.12 is that it allows to use the (very explicit) stack $L y$ in order to compute the Ext-algebra (9).

Assume that $y$ is a smooth scheme which for simplicity we shall also assume to be affine. Then $L y$ is just the dg-scheme $\operatorname{Spec}\left(\operatorname{Sym}_{\mathcal{O}_{y}} T^{*} y[1]\right)$. Since we have

$$
\operatorname{Ext}_{\left.\left.\operatorname{Sym}_{\mathcal{O} y} T^{*}[1]\right]\right)}^{*}\left(\mathcal{O}_{y}, \mathcal{O}_{y}\right)=\operatorname{Sym}_{\mathcal{O}_{y}}(T y[-2]) \text {, }
$$

we see that (with grading disregarded) $\mathbb{C}\left[\mathcal{M}_{H}\right]=\mathbb{C}\left[T^{*} y\right]$ which is what we should have in this case. In fact, if we want to rembember the grading we see that the homological grading on the RHS goes to grading coming from dilation of the cotangent fibers on the LHS. Recall that writing $X$ as $T^{*} y$ is an additional structure which is precisely the one required in order to make all the categories $\mathbb{Z}$-graded (as opposed to $\mathbb{Z}_{2}$-graded; note also that the grading on $\operatorname{Sym}_{\mathcal{O}_{y}}(T y[-2]$ ) is even, so the corresponding $\mathbb{Z}_{2}$-grading is trivial).

Let us now compute $\mathbb{C}\left[\mathcal{M}_{C}\right]$ in this case. Since $y$ is an affine scheme, it follows that $\operatorname{Maps}(\mathcal{D}, y)$ is a closed subscheme in the ind-scheme $\operatorname{Maps}\left(\mathcal{D}^{*}, y\right)$, so $\operatorname{Ext}^{*}\left(\mathcal{F}_{C}, \mathcal{F}_{C}\right)$ is just equal to the de Rham cohomology of $\operatorname{Maps}(\mathcal{D}, y)$. Since $y$ is smooth the (evaluation at $0 \in \mathcal{D})$ map $\operatorname{Maps}(\mathcal{D}, y) \rightarrow y$ is a fiber bundle whose fibers are infinite-dimensional affine spaces. Thus it induces an isomorphism on de Rham cohomology. Hence we get $\mathbb{C}\left[\mathcal{M}_{C}\right]=H^{*}(y, \mathbb{C})=H^{*}\left(T^{*} y, \mathbb{C}\right)$. So if $y$ is connected, we see that $\mathcal{M}_{C}$ is a dg-extension of pt; moreover, if $y$ is a vector space, that $\mathcal{M}_{C}=$ pt even as dg-schemes (as was promised in subsection 4.4).

7.15. Gauge theory. Consider now the the example when $y=\mathbf{N} / G$, where $G$ is a connected reductive group and $\mathbf{N}$ is a representation of $G$.

Exercise Show that in this case the RHS of (8) is literally the same as $H_{\bullet}^{G_{\mathcal{O}}}\left(\mathcal{R}_{G, \mathbf{N}}\right)$.

So, we see that our categorical point of view recovers the definition of the Coulomb branch we gave before. Let us look at the Higgs branch. According to Conjecture 7.12 we need to understand the dg-stack

$$
L(\mathbf{N} / G)=\mathbf{N} / G \underset{\mathbf{N} / G \times \mathbf{N} / G}{\times} \mathbf{N} / G .
$$

Let us actually first assume that $\mathbf{N}$ is any smooth variety with a $G$-action. Then it is easy to see that (12) is a dg-stack which admits the following description. The action of $G$ on $\mathbf{N}$ defines a natural map of locally free $\mathcal{O}_{\mathbf{N}}$-modules

$$
\mathfrak{g} \otimes \mathcal{O}_{\mathbf{N}} \rightarrow T \mathbf{N}
$$

Consider the dual map

$$
T^{*} \mathbf{N} \rightarrow \mathfrak{g}^{*} \otimes \mathcal{O}_{\mathbf{N}}
$$

and let us regard it as two step complex of coherent sheaves on $\mathbf{N}$ where $T^{*} \mathbf{N}$ lives in degree -1 and $\mathfrak{g}^{*} \otimes \mathcal{O}_{\mathbf{N}}$ lives in degree 0 . Let us denote this complex by $K^{\bullet}$. Then $\operatorname{Sym}_{\mathcal{O}_{\mathbf{N}}}\left(K^{\bullet}\right)$ is a quasi-coherent dg-algebra on $\mathbf{N}$. 
Exercise. Show that the formal neighbourhood of $\mathbf{N} / G$ in $L(\mathbf{N} / G)$ is equivalent to the formal neighbourhood of $\mathbf{N} / G$ in $\operatorname{Spec}\left(\operatorname{Sym}_{\mathcal{O}_{\mathbf{N}}}\left(K^{\bullet}\right)\right) / G$ (note that when $G$ is trivial we just recover $\left.\operatorname{Spec}\left(\operatorname{Sym}\left(T^{*} \mathbf{N}\right)[1]\right)\right)$ as in the previous subsection).

It now follows that the RHS of (9) in our case becomes equal to the $G$-invariant part of

$$
\operatorname{Ext}_{\mathrm{Sym}_{\mathcal{O}_{\mathbf{N}}}(K \bullet)}^{*}\left(\mathcal{O}_{\mathbf{N}}, \mathcal{O}_{\mathbf{N}}\right) \text {. }
$$

Assume now for simplicity that $\mathbf{N}$ is affine. Then it is easy to see that (13) is equal to the cohomology of $\operatorname{Sym}_{\mathcal{O}_{\mathrm{N}}}\left(\left(K^{\bullet}\right)^{*}[-1]\right)$.

Exercise. Show that as a $\mathbb{Z}_{2}$-graded algebra $\operatorname{Sym}_{\mathcal{O}_{\mathrm{N}}}\left(\left(K^{\bullet}\right)^{*}[-1]\right)$ is quasiisomorphic to the algebra of functions on the dg-scheme $\mu^{-1}(0)$ where $\mu: T^{*} \mathbf{N} \rightarrow \mathfrak{g}^{*}$ is the moment map.

The exercise implies that the RHS of (9) is isomorphic to the algebra of functions on the dg-stack $\mu^{-1}(0) / G$.

7.16. Mirror symmetry in the toric case. Let us assume that we are in the situation of subsection 4.6. We set $y=\mathbb{C}^{n} / T, y^{*}=\mathbb{C}^{n} / T_{F}^{\vee}$. Combining (10) and (11) with Conjecture 7.5 we already obtain a bunch of non-trivial statements. Namely, we arrive at the following

Conjecture 7.17. For the above choice of $y$ and $y^{*}$ we have equivalences of (factorization) categories

$$
\begin{aligned}
D-\bmod \left(\operatorname{Maps}\left(\mathcal{D}^{*}, y\right)\right. & \simeq \operatorname{QCoh}\left(\operatorname{Maps}\left(\mathcal{D}_{d R}^{*}, y^{*}\right)\right) \\
D-\bmod \left(\operatorname{Maps}\left(\mathcal{D}^{*}, y^{*}\right)\right) & \simeq \operatorname{QCoh}\left(\operatorname{Maps}\left(\mathcal{D}_{d R}^{*}, y\right)\right) .
\end{aligned}
$$

A proof of this conjecture is the subject of a current work in progress of the first named author with Dennis Gaitsgory. Let us discuss the simplest example (which is already quite non-trivial).

Let us take $n=1$ and let $T$ be trivial. In other words we get $y=\mathbb{A}^{1}$. Then $y^{*}=\mathbb{A}^{1} / \mathbb{G}_{m}$. So, let us look closely at what Conjecture 7.17 says in this case.

First, $\operatorname{Maps}\left(\mathcal{D}_{d R}^{*}, y\right)=\operatorname{Maps}\left(\mathcal{D}_{d R}^{*}, \mathbb{A}^{1}\right)=H_{d R}^{*}\left(\mathcal{D}^{*}\right)=\mathbb{A}^{1} \times \mathbb{A}^{1}[-1]$. In other words, $\operatorname{Maps}\left(\mathcal{D}_{d R}^{*}, \boldsymbol{y}\right)=\operatorname{Spec}(\mathbb{C}[x, \varepsilon])$ where $\operatorname{deg}(x)=0, \operatorname{deg}(\varepsilon)=-1$ (we consider it as a dg-algebra with trivial differential). The category $\mathcal{C}_{C}(y)$ is then just the derived category of dg-modules over this algebra. More precisely, it is the QCoh version of this derived category - we again refer the reader to Chapter II of [GaiRo]. The object $\mathcal{F}_{C}$ corresponds to the dg-module $\mathbb{C}[x]$ (on which $\varepsilon$ acts trivially) in degree 0.

We claim that in this case the category $\mathrm{QCoh}\left(\operatorname{Maps}\left(\mathcal{D}_{d R}^{*}, \mathbb{A}^{1}\right)\right)$ is equivalent to $D$-mod $\left(\operatorname{Maps}\left(\mathcal{D}^{*}, \mathbb{A}^{1}\right)\right)$ even as a $\mathbb{Z}$-graded category. We are not in a position to give a rigorous proof here, since for this we'll need to spell out careful definitions of both categories, and that goes beyond the scope of these notes. Let us give some 
examples of objects which go to one another under the above equivalence. First, the object $\mathcal{F}_{C} \in D-\bmod \left(\operatorname{Maps}\left(\mathcal{D}^{*}, \mathbb{A}^{1}\right)\right)$ is described as follows. Let $i_{n}: \mathcal{O} \rightarrow \mathcal{K}$ be the embedding which sends $f$ to $z^{n} f$ (here $n \in \mathbb{Z}$ ). Then we have

$$
\mathcal{F}_{C}\left(\mathbb{A}^{1} / \mathbb{G}_{m}\right)=\bigoplus_{n \in \mathbb{Z}}\left(i_{n}\right)_{*} \mathcal{O}
$$

Warning. To understand this object carefully one really needs to spell out the definition. Let us mention the problem one has to fight with. It is intuitively clear that we have a $\mathbb{Z}$-action on $\mathcal{K}$ such that $n \in \mathbb{Z}$ sends $f(z)$ to $z^{n} f(z)$. On the other hand, assume that $n \geq 0$. Then $z^{n} \mathcal{O}$ has codimension $n$ in $\mathcal{O}$ (although one is obtained from the other by means of the $\mathbb{Z}$-action). This problem is in fact not as serious as it might seem at the first glance - it just shows that the actual definition of $D$-modules on $\mathcal{K}$ (or even on $\mathcal{O}$ ) must take into account certain homological shifts.

Having the above warning in mind, it is easy to see that $\operatorname{Ext}^{*}\left(\mathcal{F}_{C}, \mathcal{F}_{C}\right)=\mathbb{C}[x, y]$ where $\operatorname{deg}(x)=0, \operatorname{deg}(y)=2$. On the other hand, we also have

$$
\operatorname{Ext}_{\mathbb{C}[x, \varepsilon]}^{*}(\mathbb{C}[x], \mathbb{C}[x])=\mathbb{C}[x, y],
$$

which matches our expectations.

Here is another example. Consider the module $\mathbb{C}$ over $\mathbb{C}[x, \varepsilon]$ (i.e. we think of it as a dg-module concentrated in degree 0 , on which $x$ acts by 1 and $\varepsilon$ acts by 0 ). Then under the above equivalence it goes to the $D$-module $\delta$ of deltafunctions at $0 \in \mathcal{K}$ (considered as a $\mathcal{K}^{*}$-equivariant $D$-module). Note that the $\mathcal{K}^{*}$-equivariant Ext from $\delta$ to itself is the same as $H_{\mathcal{K}^{*}}^{*}(\mathrm{pt}, \mathbb{C})$. Now, homotopically $\mathcal{K}^{*}$ is equivalent to $\mathbb{C}^{\times} \times \mathbb{Z}$ and we have

$$
H_{\mathbb{C}^{\times} \times \mathbb{Z}}^{*}(\mathrm{pt}, \mathbb{C})=\mathbb{C}[y, \theta] \quad \text { where } \operatorname{deg}(y)=2, \operatorname{deg}(\theta)=1 .
$$

On the other hand the same $(\mathrm{dg})$ algebra $\mathbb{C}[y, \theta]$ is equal to $\operatorname{Ext}_{\mathbb{C}[x, \varepsilon]}^{*}(\mathbb{C}, \mathbb{C})$.

7.18. The theory $\mathcal{T}[G]$. Here is another expectation. Let $D\left(\mathrm{Gr}_{G}\right)^{\text {Hecke }}$ denote the derived category of Hecke eigen-modules on $\mathrm{Gr}_{G}$, i.e. $D$-modules which are also right modules for the algebra $\mathcal{A}_{R}$.

Conjecture 7.19. The category $\mathcal{C}_{C}(\mathcal{T}[G])$ is the category $D\left(\mathrm{Gr}_{G}\right)^{\text {Hecke }}$ and $\mathcal{F}_{C}=$ $\mathcal{A}_{R}$.

Let us combine it with (10). In the case when $G=\operatorname{GL}(n)$ the theory $\mathcal{T}[G]$ does in fact come from a smooth stack $y$; here

$$
y=\left(\prod_{i=1}^{n-1} \operatorname{Hom}\left(\mathbb{C}^{i}, \mathbb{C}^{i+1}\right)\right) / \prod_{i=1}^{n-1} \operatorname{GL}(i)
$$

(note that $y$ still has an action of GL $(n)$ ). So, from (10) we get another construction of $\mathcal{C}_{C}$ which should be equivalent to the one from 7.19. 
It is in fact easy to construct a functor in one direction. Namely, let $\mathcal{C}$ be a category with a $D$-module action of some group $G$; let also $\mathcal{F}$ be a $G_{\mathcal{O}^{-}}$-equivariant object. Then $\mathcal{F}$ defines a functor $\mathrm{C} \rightarrow D$-mod $\left(\mathrm{Gr}_{G}\right)$. Moreover, this functor sends $\mathcal{F}$ to a ring object $\mathcal{A}_{\mathcal{F}}$ and the above functor can be upgraded to a functor from $\mathcal{C}$ to $\mathcal{A}_{\mathcal{F}}$-modules in $D$-mod $\left(\mathrm{Gr}_{G}\right)$. Namely, this functor sends every $\mathcal{G}$ to the $D$-module on $\operatorname{Gr}_{G}$ whose !-stalk at some $g$ is equal to $\operatorname{RHom}\left(\mathcal{F}^{g}, \mathcal{G}\right)$. In our case we take $\mathcal{C}$ to be the category of $D$-modules on $\operatorname{Maps}\left(\mathcal{D}^{*}, y\right)$ and take $\mathcal{F}=\mathcal{F}_{C}$. Then the above functor sends $\mathcal{F}$ to $\mathcal{A}_{R}$ (this is essentially proved in [BFN3]).

Note that for $G=\operatorname{GL}(n)$ the theory $\mathcal{T}[G]$ is supposed to be self-dual (with respect to mirror symmetry procedure). Hence it follows that in this case the category $\mathcal{C}_{C}$ should be equivalent to $\mathcal{C}_{H}$. Therefore, it is natural to expect that the category $\mathrm{QCoh}\left(\operatorname{Maps}\left(\mathcal{D}_{d R}^{*}, y\right)\right)$ is equivalent to $D\left(\operatorname{Gr}_{\mathrm{GL}(n)}\right)^{\text {Hecke }}$. However, we expect that it is actually wrong as stated - the reason is the warning from subsection 7.8. However, we do believe in the following

Conjecture 7.20. Let $y$ be as in (14). Then the category $\operatorname{Ind} \operatorname{Coh}\left(\operatorname{Maps}\left(\mathcal{D}_{d R}^{*}, y\right)\right)$ is equivalent to $D\left(\operatorname{Gr}_{\mathrm{GL}(n)}\right)^{\text {Hecke }}$.

Here is (an equivalent) variant of this conjecture. Note that the action of the group $\operatorname{GL}(n)$ on $y$ gives rise to an action of the same group on $\operatorname{Maps}\left(\mathcal{D}_{d R}^{*}, y\right)$. Hence we can consider the category $\mathrm{QCoh}\left(\operatorname{Maps}\left(\mathcal{D}_{d R}^{*}, y\right) / \operatorname{GL}(n)\right)$. This category admits a natural action of the tensor category $\operatorname{Rep}(\operatorname{GL}(n))$. Note that the geometric Satake equivalence also gives rise to an action of $\operatorname{Rep}(\operatorname{GL}(n))$ on $D$-mod $\left(\operatorname{Gr}_{\mathrm{GL}(n)}\right)$ (the action is by convolution with spherical $D$-modules on the right).

Conjecture 7.21. The categories $\operatorname{Ind} \operatorname{Coh}\left(\operatorname{Maps}\left(\mathcal{D}_{d R}^{*}, y\right) / \operatorname{GL}(n)\right)$ and $D-\bmod \left(\operatorname{Gr}_{\mathrm{GL}(n)}\right)$ are equivalent as module categories over $\operatorname{Rep}(\mathrm{GL}(n))$.

In the paper $[\mathrm{BF}]$ we prove a weaker version of Conjecture 7.21 for GL(2) (in particular, the version of Conjecture 7.21 proved in $[\mathrm{BF}]$ is sufficient in order to explain why we need IndCoh and not QCoh in the formulation).

7.22. G-symmetry and gauging. Let us now address the following question. Let $\mathcal{T}$ be a theory acted on by a (reductive) algebraic group $G$. What kind of structures does this action imply in terms of the categories $\mathcal{C}_{H}, \mathcal{C}_{C}$ ?

To answer this question, we need to recall two general notions. First, given a category $\mathcal{C}$ and a group ind-scheme $\mathcal{G}$ there is a notion strong or infinitesimally trivial $\mathcal{G}$-action on $\mathcal{C}$ (cf. [Gai2]). The main example of such an action is as follows: given a pre-stack $\mathcal{S}$ with a $\mathcal{G}$-action, the group $\mathcal{G}$ acts strongly on the (derived) category of $D$-modules on $\mathcal{S}$. If one replaces $D$-modules by quasi-coherent sheaves, one gets the notion of weak $G$-action on a category $\mathcal{C}$. Given a category $\mathcal{C}$ with a strong $\mathcal{G}$-action one can define the category of strongly equivariant objects in $\mathcal{C}$ (cf. [Gai2, page 4]); we shall denote this category by $\mathfrak{C}^{\mathcal{G}}$. 
On the other hand, for a stack $z$ and a (dg-)category $\mathcal{C}$ there is a notion of " $\mathcal{C}$ living over Z" (cf. [Gai1]). This simply means that the category QCoh(Z) (which is a tensor category) acts on $\mathcal{C}$. Given a geometric point $z$ of $z$ we can consider the fiber $\mathcal{C}_{z}$ of $\mathcal{C}$ at $z$. This category always has a weak action of the group Aut $_{z}$ of automorphisms of the point $z$.

Now we can formulate an (approximate) answer to the above question. Namely, we expect that a $G$-action on $\mathcal{T}$ should produce the following structures:

(1) A category $\mathcal{C}_{H}(G, \mathcal{T})$ which lives over $\operatorname{LocSys}_{G}\left(\mathcal{D}^{*}\right)$ endowed with an equivalence

$$
\mathcal{C}_{H}(G, \mathcal{T})_{\text {Triv }} \simeq \mathcal{C}_{H}(T){ }^{11}
$$

Here Triv stands for the trivial local system.

(2) A strong $G(\mathcal{K})=\operatorname{Maps}\left(\mathcal{D}^{*}, G\right)$-action on the category $\mathcal{C}_{C}(\mathcal{T})$.

Note that $G$ is the group of automorphisms of the trivial local system. Hence (1) implies that a $G$-action on $\mathcal{T}$ yields a weak action of $G$ on $\mathcal{C}_{H}(\mathcal{T})$.

Exercise. Show that this action extends to a weak action of $L G$ (which is a dg-extension of $G$ ) on $\mathcal{C}$.

The reader must be warned that a weak action of $G$ or even of $L G$ on $\mathcal{C}_{H}(\mathcal{T})$ is a very small amount of data: for example, it is not sufficient in order to reconstruct $\mathcal{C}_{H}(G, \mathcal{T})$.

Recall now that if a group $G$ acts on a theory $\mathcal{T}$ then we can form the corresponding gauge theory $\mathcal{T} / G$. Then we expect that

$$
\mathcal{C}_{H}(\mathcal{T} / G)=\mathcal{C}(G, \mathcal{T}) ; \quad \mathcal{C}_{C}(\mathcal{T} / G)=\mathcal{C}_{C}(\mathcal{T})^{G(\mathcal{K})} .
$$

Let us now go back to the case $\mathcal{T}=\mathcal{T}(\mathcal{y})$. In this case an action of $G$ on $y$ yields an action of $G$ on $\mathcal{T}(y)$. In this case we expect that $\mathcal{T}(y) / G=\mathcal{T}(y / G)$. Let us discuss the compatibility of this statement with above categorical structures. First, an action of $G$ on $y$ gives rise to an action of $G(\mathcal{K})$ on $\operatorname{Maps}\left(\mathcal{D}^{*}, y\right)$, hence a strong action on $D-\bmod \left(\operatorname{Maps}\left(\mathcal{D}^{*}, y\right)\right)$. Moreover, $D-\bmod \left(\operatorname{Maps}\left(\mathcal{D}^{*}, y\right)\right)^{G(\mathcal{K})}=$ $D-\bmod \left(\operatorname{Maps}\left(\mathcal{D}^{*}, y / G\right)\right)$ which is compatible with the 2 nd equation of $(15)$. On the other hand, it is easy to see that the category $\operatorname{QCoh}\left(\operatorname{Maps}\left(\mathcal{D}_{d R}^{*}, y / G\right)\right)$ lives over $\mathrm{QCoh}\left(\operatorname{LocSys}_{G}\left(\mathcal{D}^{*}\right)\right)$ and its fiber over Triv is $\mathrm{QCoh}\left(\operatorname{Maps}\left(\mathcal{D}_{d R}^{*}, y\right)\right)$ which is compatible with the first equation of (15).

7.23. S-duality and local geometric Langlands. This subsection is a somewhat side topic: here we would like to mention a possible connection of the above discussion with (conjectural) local geometric Langlands correspondence. A reader who is not interested in the subject is welcome to skip this subsection.

The local geometric Langlands duality predicts the existence of an equivalence $\mathbf{L}_{G}$ between the ( $\infty$-) category of (dg-) categories with strong $G(\mathcal{K})$-action and the

\footnotetext{
${ }^{11}$ Again, the reader should keep in mind subsection 7.8.
} 
$(\infty-)$ category of $(\mathrm{dg}-)$ categories over $\mathrm{QCoh}\left(\operatorname{LocSys}_{G^{\vee}}\left(\mathcal{D}^{*}\right)\right)$ (as was already mentioned earlier in these notes we are going to ignore higher categorical structures, which are in fact necessary in order to discuss these things rigorously). ${ }^{12}$

Let us now recall that given a theory $\mathcal{T}$ with a $G$-action one expects the existence of the $S$-dual theory $\mathcal{T}^{\vee}$ with a $G^{\vee}$-action. Thus we see that we get a category $\mathcal{C}_{C}\left(\mathcal{T}^{\vee}\right)$ with a strong $G^{\vee}(\mathcal{K})$-action and a category $\mathcal{C}_{H}\left(\mathcal{T}^{\vee} / G^{\vee}\right)$ which lives over $\operatorname{LocSys}_{G^{\vee}}\left(\mathcal{D}^{*}\right)$.

Conjecture 7.24. We have natural equivalences

$$
\mathbf{L}_{G}\left(\bigodot_{C}(\mathcal{T})\right) \simeq \mathcal{C}_{H}\left(\mathcal{T}^{\vee} / G^{\vee}\right) ; \quad \mathbf{L}_{G^{\vee}}\left(\bigodot_{C}\left(\mathcal{T}^{\vee}\right)\right) \simeq \mathcal{C}_{H}(\mathcal{T} / G)
$$

Recall now formula (2):

$$
\mathcal{T}^{\vee}=((\mathcal{T} \times \mathcal{T}[G]) / G)^{*}
$$

In particular, we can apply it to $\mathcal{T}$ being the trivial theory; in this case we get that the group $G^{\vee}$ should act on the theory $(\mathcal{T}[G] / G)^{*}$. Let $\mathcal{C}_{G}=\mathcal{C}_{C}\left((\mathcal{T}[G] / G)^{*}\right)$. Then this category should have a strong action of $G^{\vee}(\mathcal{K})$. On the other hand, $\mathcal{C}_{G}=\mathcal{C}_{H}(\mathcal{T}[G] / G)$, so in addition it should live over $\operatorname{LocSys}_{G}\left(\mathcal{D}^{*}\right)$ (these two structures should commute in the obvious way). We expect that $\mathcal{C}_{G}$ is the universal Langlands category for $G^{\vee}$, i.e. that for any other category $\mathcal{\complement}$ with $G^{\vee}(\mathcal{K})$ action we have

$$
\mathbf{L}_{G^{\vee}}(\mathcal{C})=\mathcal{C} \underset{G^{\vee}(\mathcal{K})}{\otimes} \mathcal{C}_{G} \cdot{ }^{13}
$$

In particular, if $G=\mathrm{GL}(n)$ then we see that the universal Langlands category $\mathcal{C}_{\mathrm{GL}(n)}$ is expected to be equivalent to $\mathrm{QCoh}\left(\operatorname{Maps}\left(\mathcal{D}_{d R}^{*}, y / G\right)\right)$ where $y$ is given by (14). Note that in this realization the fact that this category lives over $\operatorname{LocSys}_{\mathrm{GL}(n)}\left(\mathcal{D}^{*}\right)$ is clear, but the action of $\mathrm{GL}(n, \mathcal{K})$ is absolutely not obvious: we don't know how to construct it.

Again, it must be noted that the notion of universal Langlands category is not precise since as was mentioned above the correct formulation of the local geometric Langlands conjecture involves a modification of the notion of category over $\operatorname{LocSys}_{G}\left(\mathcal{D}^{*}\right)$. But at least we believe that the above description of the universal Langlands category is true as stated over the locus of irreducible local systems.

7.25. Quantization. Let us now discuss the categorical structures which give rise to the quantizations (and thus to Poisson structures) of the algebras $\mathbb{C}\left[\mathcal{M}_{H}\right]$ and $\mathbb{C}\left[\mathcal{M}_{C}\right]$. Let us first look at the latter one. The $\operatorname{space} \operatorname{Maps}\left(\mathcal{D}^{*}, y\right)$ has a

\footnotetext{
${ }^{12}$ It is known that this is only an approximate conjecture. The correct conjecture (due to A. Arinkin) requires a (rather tricky) modification of the notion category over $\operatorname{LocSys}_{G}\left(\mathcal{D}^{*}\right)$ (which again has to do with the difference between QCoh and IndCoh).

${ }^{13}$ Such a tensor product does make sense as long as we live in the world of dg-categories.
} 
natural action of the multiplicative group $\mathbb{G}_{m}$ (which acts on $\mathcal{D}^{*}$ by loop rotation). Thus the category $\mathcal{C}_{C}(y)$ admits a natural deformation: the category $D$-mod $\mathbb{G}_{m}\left(\operatorname{Maps}\left(\mathcal{D}^{*}, y\right)\right)$ of $\mathbb{G}_{m}$-equivariant $D$-modules. The object $\mathcal{F}_{C}$ deforms naturally to an object of $D-\bmod _{\mathbb{G}_{m}}\left(\operatorname{Maps}\left(\mathcal{D}^{*}, y\right)\right)$ and thus we can set

$$
\mathbb{C}_{\hbar}\left(\mathcal{M}_{C}\right)=\operatorname{Ext}_{D-\bmod _{\mathbb{G}_{m}}\left(\operatorname{Maps}\left(\mathcal{D}^{*}, y\right)\right)}\left(\mathcal{F}_{C}, \mathcal{F}_{C}\right)
$$

Here $\hbar$ as before is a generator of $H_{\mathbb{G}_{m}}^{*}(\mathrm{pt}, \mathbb{C})$.

What about the quantization of $\mathcal{M}_{H}$ ? As before we need to look for a oneparameter deformation of the pair $\left(\mathcal{C}_{H}, \mathcal{F}_{H}\right)$. Here again the action of the multiplicative group $\mathbb{G}_{m}$ on $\mathcal{D}$ and on $\mathcal{D}^{*}$ gives rise to an action of $\mathbb{G}_{m}$ on the category $\mathcal{C}_{H}(y)$; we claim that this action is strong (this is related to the fact that we work with maps from $\mathcal{D}_{d R}^{*}$ rather than with maps from $\left.\mathcal{D}^{*}\right)$. Thus it makes sense to consider the category of strongly equivariant objects in $\mathcal{C}_{H}(y)$ (cf. again [Gai2, page 4]). The Ext-algebra of (the natural analog of) the object $\mathcal{F}_{H}$ in this category is again a non-commutative algebra over $\mathbb{C}[\hbar]$ which is a quantization of $\mathbb{C}\left[\mathcal{M}_{H}\right]$.

Note that since in both cases we use the action of the multiplicative group on $\mathcal{D}^{*}$, it follows that the deformed categories are no longer factorisation categories, so the corresponding Ext-algebras no longer have factorisation structure. This is why they have a chance to become non-commutative (at least the Remark after (9) does not apply here).

7.26. Holomorphic-topological twist. We have learned the main ideas of this subsection from K. Costello. So far we discussed the two topological twists of a given theory completely independently of each other. However, in fact in physics both the $C$-twist and the $H$-twist appear as one-parametric families of equivalent twists. In addition, both families have the same limiting point, where the theory is no longer topological (it becomes holomorphic-topological, cf. [ACMV]; roughly speaking it means that, for example, for a 3-manifold $M$ the partition function $Z(M)$ is well-defined if one fixes some additional structure on $M$ which locally makes it look like a product of a complex curve $\Sigma$ and a 1-manifold $I$ ). The category of line operators in the holomorphic-topological theory is still well-defined. As a result we come to the following conclusion:

Conclusion: There should exists a factorisation category $\mathcal{C}$ with an object $\mathcal{F}$ and two $\mathbb{Z}$-gradings such that

(1) The two $\mathbb{Z}$-gradings yield the same $\mathbb{Z}_{2}$-grading.

(2) The pair $\left(\mathcal{C}_{C}, \mathcal{F}_{C}\right)$ is a deformation of the pair $(\mathcal{C}, \mathcal{F})$. This deformation preserves the 1 st grading on $\mathcal{C}$.

(3) The pair $\left(\mathcal{C}_{H}, \mathcal{F}_{H}\right)$ is a deformation of the pair $(\mathcal{C}, \mathcal{F})$. This deformation preserves the 2 nd grading on $\mathcal{C}$. 
Let us describe a suggestion for the category $\mathcal{C}(y)$ and the object $\mathcal{F}(y)$. We would like to set

$$
\mathcal{C}(y)=\mathrm{QCoh}\left(T^{*} \operatorname{Maps}\left(\mathcal{D}^{*}, y\right)\right) .
$$

Here there are some technical problems: the $\operatorname{stack} T^{*} \operatorname{Maps}\left(\mathcal{D}^{*}, y\right)$ is very essentially infinite-dimensional, so studying quasi-coherent sheaves on it is more difficult than before. Let us assume that it is possible though and let us discuss (1)-(3) in this case. First, we need two gradings. The first grading is simply the homological grading on QCoh. The second grading is the combination of the homological grading and the grading coming from $\mathbb{C}^{\times}$-action on $T^{*} \operatorname{Maps}\left(\mathcal{D}^{*}, y\right)$ (which dilates the cotangent fibers) multiplied by two (so the two grading manifestly yield the same $\mathbb{Z}_{2}$-grading).

Now the category of $D$-modules on $\operatorname{Maps}\left(\mathcal{D}^{*}, y\right)$ is clearly a deformation of $\mathrm{QCoh}\left(T^{*} \operatorname{Maps}\left(\mathcal{D}^{*}, y\right)\right)$. On the other hand, it is less clear how to deform the category $\mathrm{QCoh}\left(T^{*} \operatorname{Maps}\left(\mathcal{D}^{*}, y\right)\right)$ to $\mathrm{QCoh}\left(\operatorname{Maps}\left(\mathcal{D}_{d R}^{*}, y\right)\right)$. We plan to address these issues in a future publication.

\section{REFERENCES}

[ACMV] M. Aganagic, K. Costello, J. McNamara, C. Vafa, Topological ChernSimons/Matter Theories, arxiv:1706.09977.

[ArGa] D. Arinkin, and D. Gaitsgory, Singular support of coherent sheaves and the geometric Langlands conjecture, Selecta Math. (N.S.) 21 (2015), no. 1, 1-199.

[At] M. F. Atiyah, Topological quantum field theories, Inst. Hautes Études Sci. Publ. Math. 68 (1988), 175-186.

[ABG] S. Arkhipov, R. Bezrukavnikov, V. Ginzburg, Quantum groups, the loop Grassmannian, and the Springer resolution, J. Amer. Math. Soc. 17 (2004), no. 3, 595-678.

[BD] A. Beilinson, V. Drinfeld, Quantization of Hitchin's integrable system and Hecke eigensheaves, available at http://www.math.uchicago.edu/ mitya/langlands.html (2000).

[BeFi] R. Bezrukavnikov, M. Finkelberg, Equivariant Satake category and KostantWhittaker reduction, Moscow Math. J. 8 (2008), no. 1, 39-72.

[BiDa] R. Bielawski, A. S. Dancer, The geometry and topology of toric hyperkähler manifolds, Comm. Anal. Geom. 8 (2000), no. 4, 727-760.

[Br] T. Braden, Hyperbolic localization of intersection cohomology, Transform. Groups 8 (2003), 209-216.

[BPW] T. Braden, N. Proudfoot, B. Webster, Quantizations of conical symplectic resolutions I: local and global structure, Astérisque 384 (2016), 1-73.

[BLPW] T. Braden, A. Licata, N. Proudfoot, B. Webster, Quantizations of conical symplectic resolutions II: category $O$ and symplectic duality, Astérisque 384 (2016), $75-179$.

[BF] A. Braverman and M. Finkelberg, A quasi-coherent description of the the category of $D-\bmod \left(G r_{G L(n)}\right)$, arXiv:1809.10774.

[BFG] A. Braverman, M. Finkelberg, D. Gaitsgory, Uhlenbeck spaces via affine Lie algebras, Progr. Math. 244, Birkhäuser, Boston (2006), 17-135; Erratum, arxiv:0301176v4. 
[BFN1] A. Braverman, M. Finkelberg, H. Nakajima, Towards a mathematical definition of 3-dimensional $\mathcal{N}=4$ gauge theories, II, arXiv:1601.03586.

[BFN2] A. Braverman, M. Finkelberg, H. Nakajima, Coulomb branches of $3 d \mathcal{N}=4$ quiver gauge theories and slices in the affine Grassmannian (with appendices by Alexander Braverman, Michael Finkelberg, Joel Kamnitzer, Ryosuke Kodera, Hiraku Nakajima, Ben Webster, and Alex Weekes), arXiv:1604.03625.

[BFN3] A. Braverman, M. Finkelberg, H. Nakajima, Ring objects in the equivariant derived Satake category arising from Coulomb branches (with appendix by Gus Lonergan), arXiv:1706:02112.

[BFN4] A. Braverman, M. Finkelberg, H. Nakajima, Line bundles over Coulomb branches, arXiv:1805.11826.

[CHZ] S. Cremonesi, A. Hanany, A. Zaffaroni, Monopole operators and Hilbert series of Coulomb branches of $3 d \mathcal{N}=4$ gauge theories, JHEP 1401 (2014), 005.

[DG] V. Drinfeld, D. Gaitsgory, On a theorem of Braden, Transform. Groups 19 (2014), $313-358$.

[FiMi] M. Finkelberg, I. Mirković, Semi-infinite flags. I. Case of global curve $\mathbb{P}^{1}$, Amer. Math. Soc. Transl. Ser. 2, 194, Amer. Math. Soc., Providence, RI (1999), 81-112.

[GW] D. Gaiotto and E. Witten, $S$-duality of boundary conditions in $\mathcal{N}=4$ super YangMills theory, Adv. Theor. Math. Phys. 13 (2009), no. 3, 721-896.

[Gai1] D. Gaitsgory, The notion of category over an algebraic stack, arXiv:math/0507192.

[Gai2] D. Gaitsgory, Groups Acting on Categories, http://www.math.harvard.edu/ gaitsgde/ grad_2009/SeminarNotes/April6(GrpActCat).pdf

[Gai3] D. Gaitsgory, Day VI, Talk 1. OPERS, Hebrew University school on geometric Langlands, March 2014.

[GaiRo] D. Gaitsgory, N. Rozenblyum, A study in derived algebraic geometry, Mathematical surveys and monographs 221, American Mathematical Society, Providence, RI (2017).

[Gi1] V. Ginzburg, Perverse sheaves on a loop group and Langlands' duality, arXiv:alggeom/9511007.

[Gi2] V. Ginsburg, Perverse sheaves and $\mathbb{C}^{*}$-actions, J. Amer. Math. Soc. 4 (1991), no. 3, 483-490.

[GK] V. Ginzburg, D. Kazhdan, Construction of symplectic varieties arising in 'Sicilian theories', in preparation.

[GiRi] V. Ginzburg, S. Riche, Differential operators on $G / U$ and the affine Grassmannian, J. Inst. Math. Jussieu 14 (2015), no. 3, 493-575.

[Gr] A. Grothendieck, Sur la classification des fibrés holomorphes sur la sphère de Riemann, Amer. J. Math. 79 (1957), 121-138.

[Ka] M. Kashiwara, The flag manifold of Kac-Moody Lie algebra, Algebraic analysis, geometry and number theory (Baltimore, MD, 1988), Johns Hopkins Univ. Press (1989), 161-190.

[KT] M. Kashiwara, T. Tanisaki, Kazhdan-Lusztig conjecture for affine Lie algebras with negative level, Duke Math. J. 77 (1995), 21-62.

[KWY] J. Kamnitzer, B. Webster, A. Weekes, O. Yacobi, Yangians and quantizations of slices in the affine Grassmannian, Algebra and Number Theory 8 (2014), no. 4, 857-893.

[Lur1] J. Lurie, On the Classification of Topological Field Theories, Current developments in mathematics, Int. Press, Somerville, MA (2008), 129-280. 
[Lur2] J. Lurie, Derived Algebraic Geometry VI: $\mathbb{E}[k]$-Algebras, http://www.math.harvard.edu/ lurie/papers/DAG-VI.pdf.

[Lus] G. Lusztig, Singularities, character formulas, and a q-analogue of weight multiplicities, Astérisque 101-102, Soc. Math. France (1983), 208-229.

[MV] I. Mirković, K. Vilonen, Geometric Langlands duality and representations of algebraic groups over commutative rings, Ann. of Math. (2) 166 (2007), no. 1, 95-143.

[MT] G. W. Moore, Y. Tachikawa, On 2d TQFTs whose values are holomorphic symplectic varieties, String-Math 2011, Proc. Sympos. Pure Math. 85, Amer. Math. Soc., Providence, RI (2012), 191-207.

[Na1] H. Nakajima, Lectures on perverse sheaves on instanton moduli spaces, IAS/Park City Math. Ser. 24, Amer. Math. Soc., Providence, RI (2017), 381-436.

[Na2] H. Nakajima, Towards a mathematical definition of Coulomb branches of 3dimensional $\mathcal{N}=4$ gauge theories, I, Adv. Theor. Math. Phys. 20 (2016), no. 3, 595-669.

[Na3] H. Nakajima, Questions on provisional Coulomb branches of 3-dimensional $\mathcal{N}=4$ gauge theories, arXiv:1510.03908.

[Na4] H. Nakajima, Introduction to a provisional mathematical definition of Coulomb branches of 3-dimensional $\mathcal{N}=4$ gauge theories, arXiv:1706.05154.

[NT] H. Nakajima, Y. Takayama, Cherkis bow varieties and Coulomb branches of quiver gauge theories of affine type A, Selecta Math. (N.S.) 23 (2017), no. 4, 2553-2633.

[Ras] S. Raskin, Chiral categories, http://math.mit.edu/ sraskin/chiralcats.pdf

[Ras-dm] S. Raskin, D-modules on infinite-dimensional varieties, http://math.mit.edu/ sraskin/dmod.pdf

[SW] N. Seiberg, E. Witten, Gauge dynamics and compactification to three dimensions, Adv. Ser. Math. Phys. 24, World Sci. Publ., River Edge, NJ (1997), 333-366.

[W] B. Webster, Koszul duality between Higgs and Coulomb categories $\mathcal{O}$, arXiv:1611.06541.

Department of Mathematics, University of Toronto and Perimeter Institute of Theoretical Physics, Waterloo, Ontario, Canada, N2L 2 Y5

Skolkovo Institute of Science and Technology;

E-mail address: braval@math.toronto.edu

National Research University Higher School of Economics, Russian FederaTION

Department of Mathematics, 6 Usacheva st., Moscow 119048;

Skolkovo Institute of Science and Technology;

Institute for Information Transmission Problems

E-mail address: fnklberg@gmail.com 